\title{
$N$-Functionalized Organolithium Compounds via Tellurium/Lithium Exchange Reaction
}

\author{
Fabricio Vargas, * Fabiano T. Toledo and João V. Comasseto \\ Instituto de Química, Universidade de São Paulo, Av. Prof. Lineu Prestes, 748, \\ 05508-000 São Paulo-SP, Brazil
}

\begin{abstract}
Um conjunto de amino teluretos foram preparados e testados em reações de troca telúrio/ lítio. Os compostos de organolítio contendo nitrogênio foram facilmente preparados utilizando $N$-Bz teluroaminas e uma combinação de $n$-butillítio/naftaleto de lítio nas reações de troca. Os correspondentes intermediários dilitiados foram capturados com vários eletrófilos, levando à formação dos respectivos produtos em bons rendimentos. A reação foi empregada na síntese de fenetilaminas.
\end{abstract}

A set of tellurium amines have been evaluated in the tellurium/lithium exchange reaction. The nitrogen-containing organolithium compounds were efficiently prepared by using $N$-Bz tellurium amines and a mixture of $n$-butyllithium and lithium naphthalenide ( $\mathrm{LiNp}$ ) for performing the exchange reaction. The corresponding dianion intermediates were trapped with a wide range of electrophiles, furnishing the corresponding products in good to excellent yields. The reaction was also employed in the synthesis of phenethylamines.

Keywords: tellurium/lithium exchange reaction, functionalized organolithium, dianion intermediate, phenethylamines

\section{Introduction}

Organolithium compounds are versatile and useful organometallics due to their excellent nucleophilic reactivity and importance in synthetic organic chemistry. ${ }^{1}$ Among them, functionalized organolithium compounds represent an important class of intermediates with the ability of transfering the functionality to an electrophilic reagent. In this way, the preparation of functionalized organolithium compounds via halogen/lithium ${ }^{2}$ and tin/ lithium ${ }^{3}$ exchange reactions represent an interesting protocol of carbon-carbon bond formation for the synthesis of polyfunctionalized molecules, as well as a wide range of natural products. For years, several groups have been interested in the study and preparation of different $\alpha-, \beta-, \gamma-, \delta$-oxygen or nitrogen-functionalized organolithium intermediates and their reaction with electrophiles. ${ }^{4,5}$ However, among these intermediates, the corresponding $\beta$-functionalized compounds, showed to be very unstable species, decomposing via $\beta$-elimination to give olefins. ${ }^{6}$

\footnotetext{
*e-mail: fvargas@iq.usp.br
}

On the other hand, organotellurium chemistry is a very broad and exciting field with many opportunities for research and development of applications in organic synthesis. ${ }^{7}$ Many different classes of organotellurium compounds have been prepared to date and successfully employed in different synthetic applications, ${ }^{8}$ as well as in the total synthesis of natural products. ${ }^{9}$ Among the several applications of organotellurium compounds, the formation of organolithium compounds via tellurium/ lithium exchange reaction represents an interesting protocol with a number of advantages over alternative methods. ${ }^{10,11}$

Our research group has been working in the preparation and application of hydroxy alkyl tellurides in the tellurium/ lithium exchange reaction. ${ }^{12}$ These compounds have been efficiently used as alternative organometallic sources of 1,4-dianion intermediates in the synthesis of diols, ${ }^{13}$ spiroketals, ${ }^{14}$ bioactive butenolides,${ }^{15}$ and in the synthesis of natural products, such as (+/-)-frontalin ${ }^{16}$ and (+)-endobrevicomin. ${ }^{17}$ In this way, based on the interesting results obtained with the application of oxygen-containing organotellurium compounds ${ }^{12-16}$ and following our current interest concerning the development of functionalized alkyl tellurides in organic synthesis, we decided to explore herein 
the application of nitrogen-containing organotellurium compounds in tellurium/lithium exchange reactions.

\section{Results and Discussion}

The preparation of the corresponding nitrogencontaining organotellurium compounds was described in our previous communication, ${ }^{18}$ via the ring-opening reaction of aziridines by tellurium nucleophiles generated in situ, as depicted in Scheme 1. All the $\beta$-organotellurium amines, bearing different steric and electronic characteristics, were obtained in up to $89 \%$ yield and under very mild conditions.

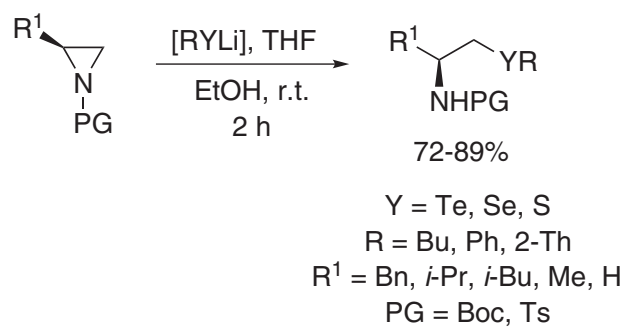

Scheme 1. Ring-opening reaction of aziridines by chalcogen nucleophiles.

The $\beta$-organotellurium compounds derived from L-phenylalanine bearing $t$-butoxycarbonyl and tosyl protecting groups were employed as model substrates in order to evaluate the formation of the nitrogenfunctionalized organolithium intermediates by tellurium/ lithium exchange reaction (Scheme 2). In this way, the corresponding alkyl tellurides were initially submitted to the conditions successfully employed by our group for hydroxy tellurides, previously used as substrates in the tellurium/lithium exchange reaction. ${ }^{13}$

According to the reaction conditions described in Scheme 2, $n$-BuLi (2 equiv.) was used to generate the dianion intermediate. When $N$-Ts $\beta$-tellurium amine was used as substrate, the $\mathrm{Te} / \mathrm{Li}$ exchange reaction could be followed by TLC (disappearance of the starting material). However, after addition of benzaldehyde to the dilithium intermediate, and even after prolonged reaction time at $-78^{\circ} \mathrm{C}$ to r.t., the formation of the expected $\gamma$-aminoalcohol could not be observed. Flash chromatography of the crude product revealed the presence of $p$-toluenesulphonamide and other side products, probably arising from a $\beta$-elimination reaction on the corresponding dilithium intermediate. The same results were observed, by using different solvents (THF, ether), temperatures $\left(-78,-20,0{ }^{\circ} \mathrm{C}\right.$, r.t.), additives such HMPA, TMEDA and LiNp (lithium naphtalenide ${ }^{19}$ ), as well as $t$-BuLi. Based on these unsucessful results obtained with the $\beta$-tellurium amines derived from L-phenylalanine in $\mathrm{Te} / \mathrm{Li}$ exchange reactions, we decided to evaluate the behaviour of a very simple class of $\beta$-tellurium amines, containing different protecting groups $(\mathrm{PG}=\mathrm{Boc}, \mathrm{Cbz}, \mathrm{Bz}$ and Ts) and only a $\mathrm{C} 2$ carbon chain between the tellurium and nitrogen moieties, aiming to minimize the possibility of $\beta$-elimination. Thus, the $N$-Boc and $N$-Cbz $\beta$-telluro amines 1a and $\mathbf{1 b}$ were prepared in 76 and $78 \%$ yields, respectively, by nucleophilic displacement of the mesylate groups of BocHNCH ${ }_{2} \mathrm{CH}_{2} \mathrm{OMs}^{20}$ and $\mathrm{CbzHNCH}_{2} \mathrm{CH}_{2} \mathrm{OMs},{ }^{21}$ using $n$-BuLi and elemental tellurium [BuTeLi] in a mixture of THF and ethanol (Scheme 3, path a). On the other hand, the $N$-Ts and $N$-Bz $\beta$-telluro amines $1 \mathbf{c}$ and $\mathbf{1 d}$ were easily obtained via the ring-opening reaction of the corresponding aziridines, ${ }^{17,22}$ using the same tellurium nucleophilic species (Scheme 3, path b).

The $\beta$-tellurium amines 1a-d were evaluated in the tellurium/lithium exchange reaction. In this way, when the $\beta$-tellurium amines containing carbamate moieties (1a, PG = Boc and $\mathbf{1 b}, \mathrm{PG}=\mathrm{Cbz}$ ) were used as substrates, we could not observe the tellurium/lithium exchange reaction by TLC, evidencing that the presence of these groups, attached to nitrogen, have a negative effect on the present exchange reaction. On the other hand, under several reaction conditions, the $\beta$-tellurium amine 1c showed a similar behaviour to the $N$-Ts $\beta$-tellurium amine presented in Scheme 2. After the reaction work up, we could only observe the presence of $p$-toluenesulphonamide and additional by-products. However, when the $N$-Bz $\beta$-tellurium amine $1 \mathbf{d}$ was employed as starting material, the tellurium/lithium exchange reaction ocurred in the presence of a mixture of $n$-butyllithium and lithium naphthalenide (LiNp), developed by Yus et al. ${ }^{23}$ for chloroamide lithiation. The corresponding $\beta$-dilithium intermediate was trapped with benzaldehyde, furnishing the corresponding $\gamma$-aminoalcohol 2a in 79\% yield (Scheme 4).

After determining the best experimental conditions to generate the corresponding $N$-Bz $\beta$-dilithium intermediate, the tellurium/lithium exchange reaction was further expanded to a broader range of electrophilic species, as

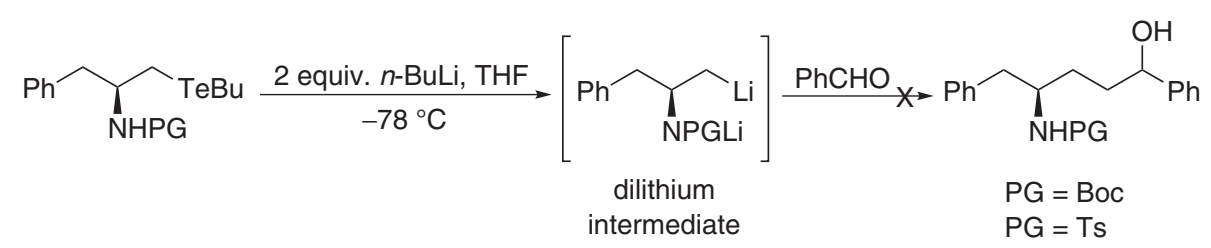

Scheme 2. Tellurium/lithium exchange reaction of $\beta$-tellurium amines bearing $t$-butoxycarbonyl and tosyl protecting groups. 


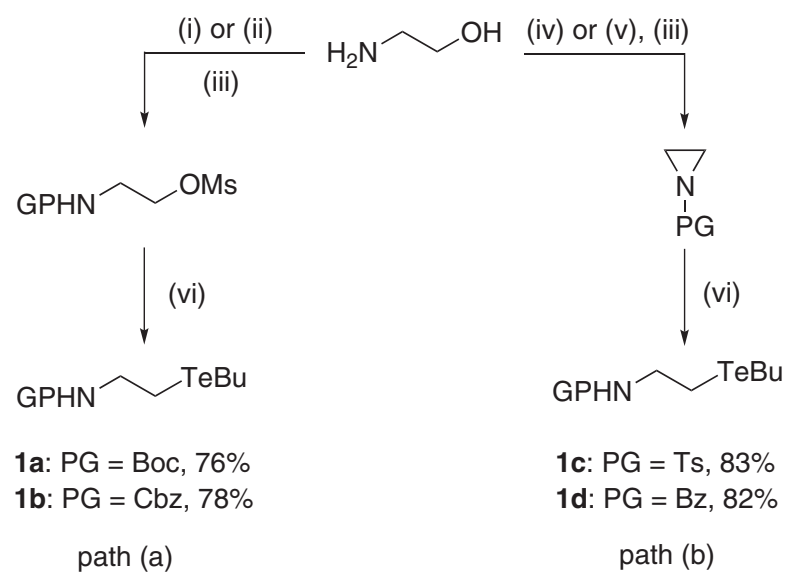

(i) $(\mathrm{Boc})_{2} \mathrm{O}, \mathrm{CH}_{3} \mathrm{CN}$, r.t., $4 \mathrm{~h}$ (ii) $\mathrm{CbzCl}, \mathrm{NaHCO}_{3}$, dioxane, r.t., $12 \mathrm{~h}$ (iii) $\mathrm{MsCl}, \mathrm{CH}_{2} \mathrm{Cl}_{2}$, r.t., overnight (iv) $\mathrm{TsCl}, \mathrm{CH}_{2} \mathrm{Cl}_{2}$, r.t., $4 \mathrm{~h}$ (v) $\mathrm{BzCl}, \mathrm{THF} / \mathrm{H}_{2} \mathrm{O}, \mathrm{Et}_{3} \mathrm{~N}$, r.t., 20 h; (vi) [BuTeLi], THF/EtOH, r.t. 2 h.

Scheme 3. Synthesis of $\beta$-tellurium amines 1a-d.

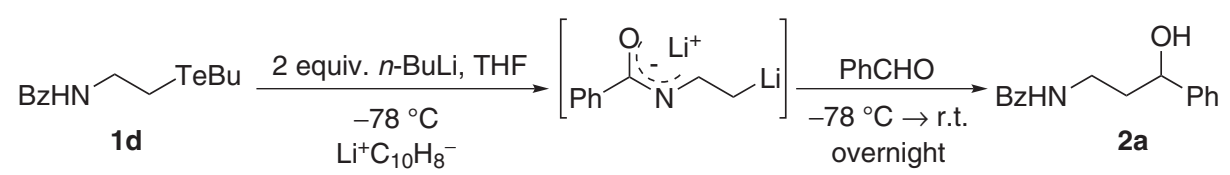

Scheme 4. Tellurium/lithium exchange reaction of $\mathbf{1 d}$.

well as to $\gamma$ - and $\delta$-tellurium amines, in order to evaluate its scope and limitations of such protocol (Table 1).

As can be seen, all the desired products were obtained in good to excellent yields. The tellurium/lithium exchange reaction was evaluated in the presence of a wide range of carbonyl compounds and the respective products were isolated in up to $82 \%$ yield (Table 1 , entry 3 ). When para, ortho, and meta tolualdehyde were employed as electrophiles, the aminoalcohols $\mathbf{2 b}$-d were obtained in a similar yield, showing that steric hindrance does not play an important role in the exchange reaction (Table 1, compare entries 2-4). The same protocol, using 2-furylaldehyde as electrophile, afforded product $2 \mathrm{e}$ in $68 \%$ yield. When aromatic aldehydes were replaced by octyl aldehyde, butyraldehyde and isobutyraldehyde the corresponding aminoalcohols $\mathbf{2 g - i}$ were obtained in yields ranging from 75 to $79 \%$ (Table 1, entries 7-9). Ketones were also successfully employed in the process. However, the tertiary alcohols $\mathbf{2} \mathbf{j}-\mathbf{l}$ were isolated in lower yields (Table $\mathbf{1}$, entries 10-12) as compared to the more reactive aldehydes. Additionally, trimethylsilylchloride and water were also employed as electrophiles, yielding the corresponding products $\mathbf{2 m}$ and $\mathbf{2 n}$ in 67 and $78 \%$ yields, respectively (Table 1, entries 13 and 14). The organolithium species derived from tellurides $\mathbf{1 e}(\mathrm{n}=2)$ and $\mathbf{1 f}(\mathrm{n}=3)$ were also efficiently trapped with benzaldehyde, furnishing the corresponding aminoalcohols $\mathbf{2 0}$ and $\mathbf{2 p}$ in good yields (Table 1, entries 15 and 16).
The present tellurium/lithium exchange reaction was also extended to prepare a class of phenethylamines (PEAs), ${ }^{24}$ that are interesting substructures for a variety of biologically important compounds, including dopamine, tyrosine, amphetamine, and adrenaline, and are also widely found as components of alkaloid natural products. ${ }^{25}$ Moreover, such compounds often serve as key building blocks in the synthesis of numerous nitrogen-containing complex molecules.

The desired products were obtained using the same reaction conditions previously developed for the exchange reaction, but using aryl halides instead of aldehydes or ketones. It should be mentioned that the reaction proceeds with comparable yields when bromine and chlorine are used as leaving groups on the aryl moiety (Table 2, entries 1 and 2). The reaction also works using brominated substrates with different electronic demands at the ortho, meta and para positions, furnishing products $\mathbf{3 b}$-e in moderate to good yields (Table 2, entries 4-7).

\section{Conclusions}

In summary, we have described the synthesis of structurally diverse $\beta$-tellurium amines that were transformed into the corresponding nitrogen-containing organolithium compounds via $\mathrm{Te} / \mathrm{Li}$ exchange reaction of $N-\mathrm{Bz}$ protected derivatives by using a mixture of $n$-butyllithium and lithium naphthalenide (LiNp). The 
Table 1. Tellurium/lithium exchange reaction of $N$-Bz $\beta$-tellurium amine 1d-f with different electrophiles

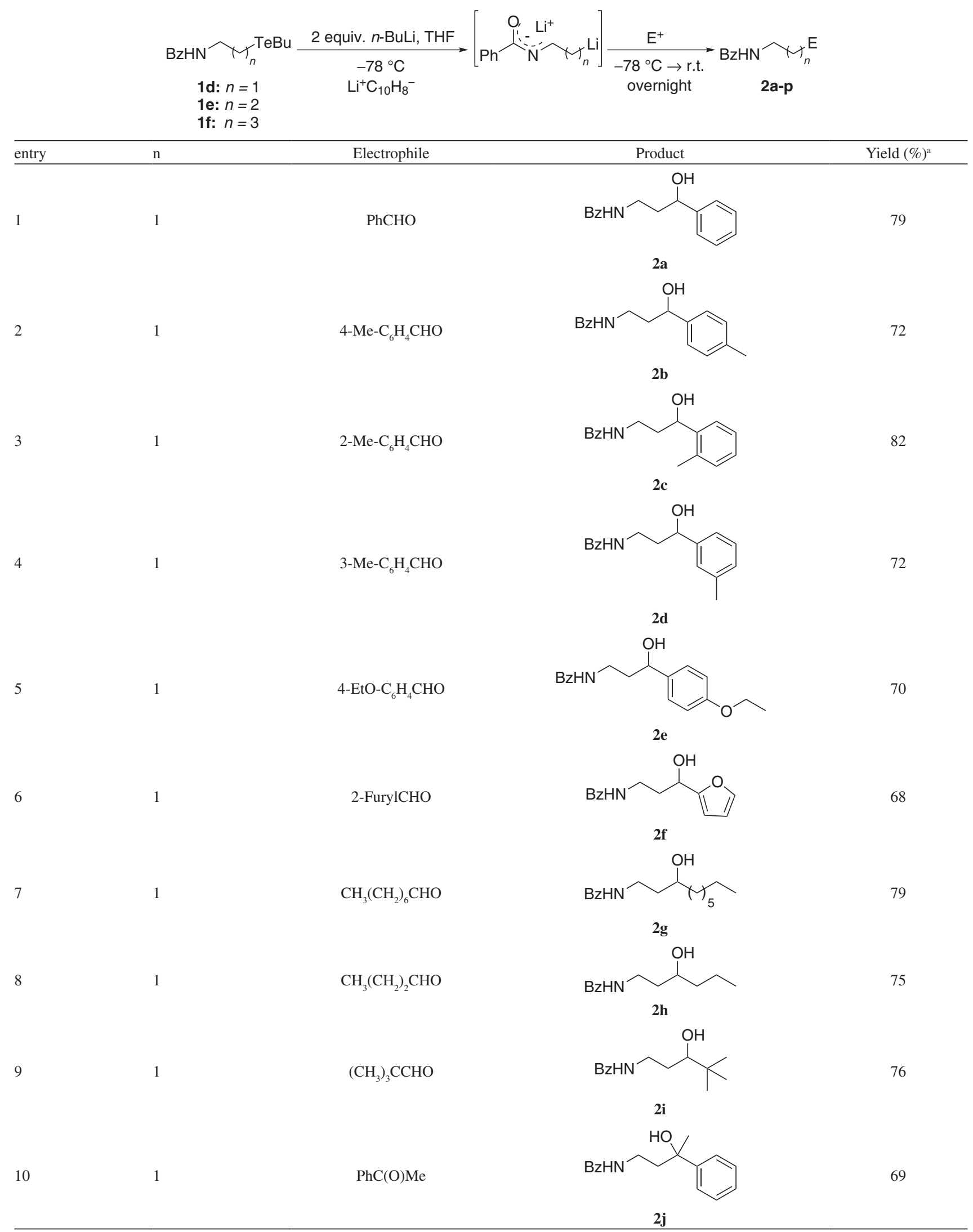


Table 1. continuation

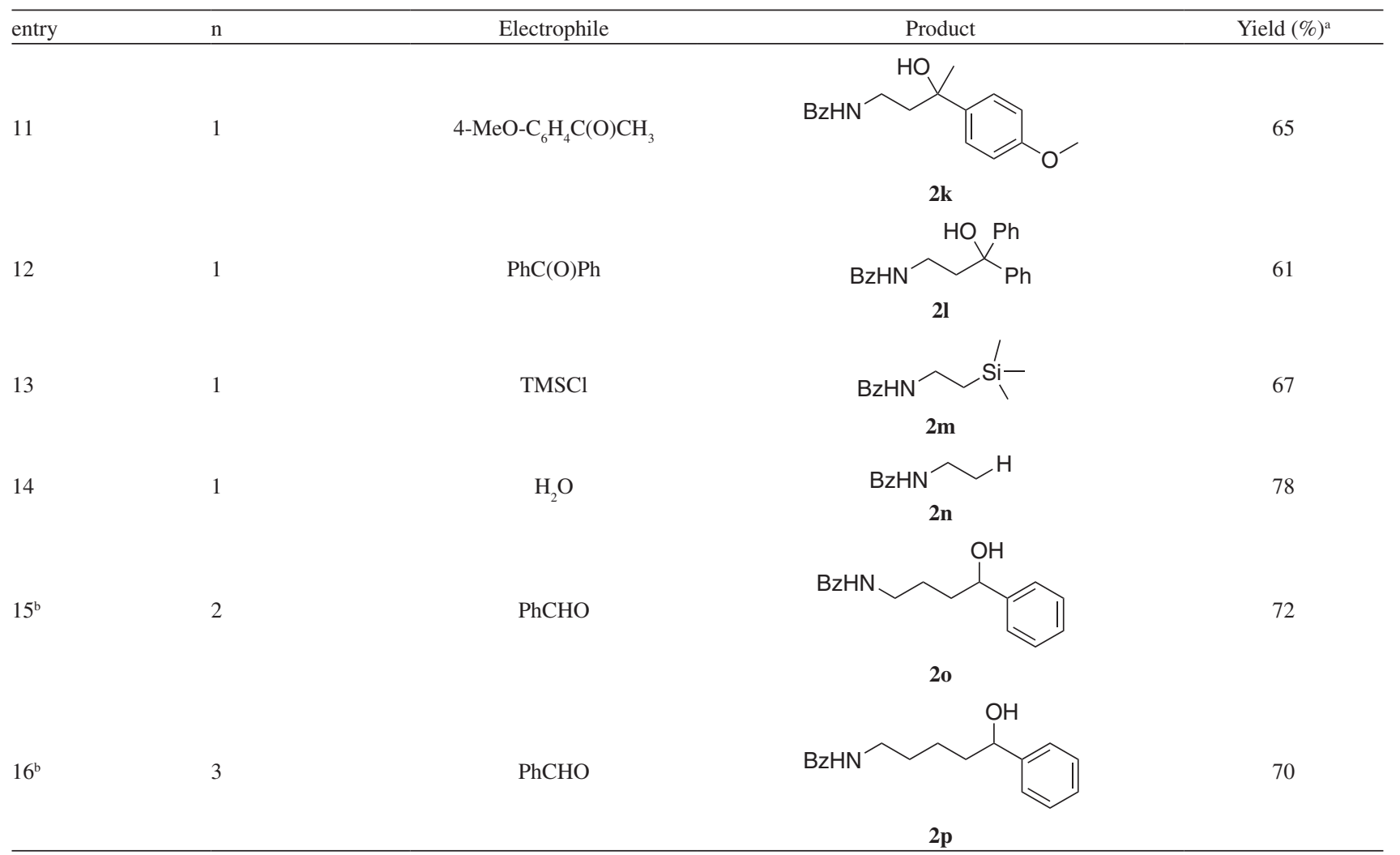
${ }^{\mathrm{a}}$ Isolated yield of the corresponding product. ${ }^{\mathrm{b}} \mathrm{The}$ corresponding $\gamma$ - and $\delta$-tellurium amines $\mathbf{1 e}$ and $\mathbf{1 f}$ were prepared by nucleophilic displacement of $\mathrm{BzHNCH}_{2} \mathrm{CH}_{2} \mathrm{CH}_{2} \mathrm{OMs}$ and $\mathrm{BzHNCH}_{2}\left(\mathrm{CH}_{2}\right)_{2} \mathrm{CH}_{2} \mathrm{OMs}$ with [BuTeLi] , respectively.

Table 2. Preparation of phenethylamines via the tellurium/lithium exchange reaction

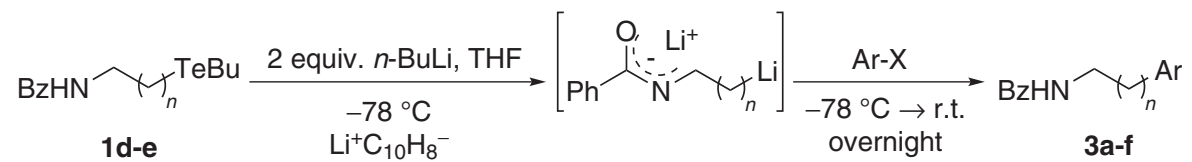

\begin{tabular}{|c|c|c|c|c|}
\hline entry & $\mathrm{n}$ & Ar-X & Product & Yield $(\%)^{\mathrm{a}}$ \\
\hline 1 & 1 & $\mathrm{PhBr}$ & $3 a$ & 65 \\
\hline 2 & 1 & $\mathrm{PhI}$ & 3a & 66 \\
\hline 3 & 1 & $\mathrm{PhCl}$ & 3a & traces \\
\hline 4 & 1 & 4-MeO- $\mathrm{C}_{6} \mathrm{H}_{4} \mathrm{Br}$ & $3 b$ & 69 \\
\hline 5 & 1 & 4-Me- $\mathrm{C}_{6} \mathrm{H}_{4} \mathrm{Br}$ & $3 \mathbf{c}$ & 66 \\
\hline
\end{tabular}


Table 2. continuation

\begin{tabular}{|c|c|c|c|c|}
\hline entry & $\mathrm{n}$ & Ar-X & Product & Yield $(\%)^{\mathrm{a}}$ \\
\hline 6 & 1 & 2-Me- $\mathrm{C}_{6} \mathrm{H}_{4} \mathrm{Br}$ & $3 d$ & 42 \\
\hline 7 & 1 & $3-\mathrm{CF}_{3}-\mathrm{C}_{6} \mathrm{H}_{4} \mathrm{Br}$ & $3 \mathrm{e}$ & 35 \\
\hline 8 & 2 & $\mathrm{PhBr}$ & $3 \mathbf{f}$ & 57 \\
\hline
\end{tabular}

asolated yield of the corresponding product.

dilithium intermediate was conveniently trapped with several electrophiles, furnishing the desired products in good to excellent yields. The reaction was also employed in the synthesis of phenethylamines and their derivatives. To the best of our knowledge this is the first time that nitrogen-containing organotellurium compounds have been successfully used as a source of $N$-functionalized organolithium compounds.

\section{Supplementary Information}

Experimental details and spectra are available free of charge at http://jbcs.sbq.org.br, as PDF file.

\section{Acknowledgments}

The authors gratefully acknowledge FAPESP and CNPq for financial support.

\section{References}

1. Wakefield, B. J.; Organolithium Methods, Academic: London, 1988.

2. Chinchila, R.; Nájera, C.; Yus, M.; Tetrahedron 2005, 61, 3139; Nájera, C.; Sansano, J. M.; Yus, M.; Tetrahedron 2003, 59, 9255; Nájera, C.; Yus, M.; Curr. Org. Chem. 2003, 7, 867.

3. Rim, C.; Son, D. Y.; ARKIVOC 2006, 265.

4. Barfoot, C. W.; Harvey, J. E.; Kenworthy, M. N.; Kilburn, J. P.; Ahmed, M.; Taylor, R. J. K.; Tetrahedron 2005, 61, 3403; Yus, M.; Gomis, J.; Eur. J. Org. Chem. 2003, 2043; Yus, M.; Ramón, D. J.; Gómez, I.; Tetrahedron 2002, 58, 5163; Yus, M.; Soler, T.; Foubelo, F.; Tetrahedron 2002, 58, 7009; Barluenga, J.; Canteli, R.-M.; Flórez, J.; J. Org. Chem. 1994, 59, 1586; Almena, J.; Foubelo, F.; Yus, M.; J. Org. Chem. 1994, 59, 3210; Ibánez, P.; Nájera, C.; Tetrahedron Lett. 1993, 34, 2003; Barluenga, J.; Montserrat, J. M.; Flórez, J.; J. Org. Chem. 1993, 58, 5976;
Barluenga, J.; Foubelo, F.; Fañanás, F. J.; Yus, M.; Tetrahedron 1989, 45, 2183; Barluenga, J.; Foubelo, F.; Fañanás, F. J.; Yus, M.; Tetrahedron Lett. 1988, 29, 2859.

5. Barluenga, J.; Pure Appl. Chem. 1990, 62, 595.

6. Barluenga, J.; Fernández-Simón, J. L.; Concéllon, J. M.; Yus, M.; J. Chem. Soc., Chem. Commun. 1986, 1665.

7. Comasseto, J. V.; Barrientos-Astigarraga, R. E.; Aldrichim. Acta 2000, 33, 66; Comasseto, J. V.; Cunha, R. L. O. R.; Clososki, G. C. In Comprehensive Organometallic Chemistry III, Crabtree, R. H., Mingos, M. P., eds., vol. 9, Elsevier: Oxford, 2007, p. 587.

8. Zeni, G.; Lüdtke, D. S.; Panatieri, R. B.; Braga, A. L.; Chem. Rev. 2006, 106, 1032.

9. Marino, J. P.; McClure, M. S.; Holub, D. P.; Comasseto, J. V.; Tucci, F. C. ; J. Am. Chem. Soc. 2002, 124, 1664 ; Zeni, G.; Panatieri, R. B.; Lissner, E.; Menezes, P. H.; Braga, A. L.; Stefani, H. A.; Org. Lett. 2001, 3, 819 ; Yang, J.; Cohn, S. T.; Romo, D.; Org. Lett. 2000, $2,763$.

10. Reich, H. J., Bevan, M. J., Gudmundsson, B. Ö., Puckett, C. L.; Angew.Chem. Int. Ed. 2002, 41, 3436; Reich, H. J., Green, D. P., Phillips, N. H., Borst, J. P., Reich, I. L.; Phosphorus, Sulfur, Silicon Relat. Elem. 1992, 67, 83.

11. For tellurium/lithium exchange reaction, see: Sugimoto, O.; Sudo, M.; Tanji, K.; Tetrahedron 2001, 57, 2133; Dabdoub, M. J.; Jacob, R. G.; Ferreira, J. T. B.; Dabdoub, V. B.; Marques, F. A.; Tetrahedron Lett. 1999, 40, 7159; Katkevics, M.; Yamaguchi, S.; Toshimitsu, A.; Tamao, K.; Organometallics 1998, 17, 5796; Inoue, T.; Atarashi, Y.; Kamble, N.; Ogawa, A.; Sonoda, N.; Synlett. 1995, 209.

12. For recent reviews in thellurium/lithium exchange reaction, see: Dos Santos, A. A.; Comasseto, J. V.; Phosphorus, Sulfur, Silicon Relat. Elem. 2008, 183, 939; Wendler, E.; Santos, A. A.; Synlett 2009, 1034.

13. Princival, J. L.; Barros, S. M. G.; Comasseto, J. V.; Dos Santos, A. A.; Tetrahedron Lett. 2005, 46, 4423.

14. Dos Santos, A. A.; Princival, J. L.; Comasseto, J. V.; Barros, S. M. G.; Neto, J. E. B.; Tetrahedron 2007, 63, 5167. 
15. Bassora, B. K.; Costa, C. E.; Gariani, R. A.; Comasseto, J. V.; Dos Santos, A. A.; Tetrahedron Lett. 2007, 48, 1485.

16. Dos Santos, A. A.; Ferrarini, R. S.; Princival, J. L.; Comasseto, J. V.; Tetrahedron Lett. 2006, 47, 8933.

17. Ferrarini, R. S.; Princival, J. L.; Comasseto, J. V.; Dos Santos, A. A.; J. Braz. Chem. Soc. 2008, 19, 811.

18. Vargas, F.; Comasseto, J. V.; J. Organomet. Chem. 2008, 694, 122.

19. Barfoot, C. W.; Harvey, J. E.; Kenworthy, M. N.; Kilburn, J. P.; Ahmed, M.; Taylor, R. J. K.; Tetrahedron 2005, 61, 3403; Barluenga, J.; Montserrat, J. M.; Flórez, J.; J. Org. Chem. 1993, 58, 5976; Barluenga, J.; Foubelo, F.; Fañanás, F. J.; Yus, M.; Tetrahedron 1989, 45, 2183; Barluenga, J.; Foubelo, F.; Fañanás, F. J.; Yus, M.; Tetrahedron Lett. 1988, 29, 2859.

20. Vliet, L. D. V.; Ellis, T.; Foley, P. J.; Liu, L.; Pfeffer, F. M.; Russell, R. A.; Warrener, R. N.; Hollfelder, F.; Waring, M. J.; J. Med. Chem. 2007, 50, 2326.

21. Townsend, C. A.; Basak, A.; Tetrahedron 1991, 47, 2591.

22. Ślebocka-Tilk, H.; Brown, R. S.; J. Org. Chem. 1987, 52, 805.

23. Barluenga, J.; Foubelo, F.; Fañanás, F. J.; Yus, M.; Tetrahedron Lett. 1988, 29, 2859.
24. For the synthesis of phenethylamines, see: Nordlander, J. E.; Payne, M. J.; Njoroge, F. G.; Balk, M. A.; Laikos, G. D.; Vishwanath, V. M.; J. Org. Chem. 1984, 49, 4107; Busacca, C. A.; Johnson, R. E.; Swestock, J.; J. Org. Chem. 1993, 58, 3299; Duddu, R.; Eckhardt, M.; Furlong, M.; Knoess, H. P.; Berger, S.; Knochel, P.; Tetrahedron 1994, 50, 2415; Hunter, C.; Jackson, R. F. W.; Rami, H. K.; J. Chem. Soc., Perkin Trans. 1 2000, 219; Rilatt, I.; Caggiano, L.; Jackson, R. F. W.; Synlett 2005, 2701; Barluenga, J.; Montserrat, J. M.; Florez, J.; J. Org. Chem. 1993, 58, 5976; Kamatani, A.; Overman, L. E.; J. Org. Chem. 1999, 64, 8743. Molander, G. A., Vargas, F.; Org. Lett. 2007, 9, 203.

25. Bentley, K. W.; Nat. Prod. Rep. 1999, 16, 367; Lednicer, Mitscher, L. A.; The Organic Chemistry of Drug Synthesis; Wiley: New York, Vol. 7, 1997.

Submitted: April 1, 2010

Published online: August 27, 2010

FAPESP has sponsored the publication of this article. 


\title{
$N$-Functionalized Organolithium Compounds via Tellurium/Lithium Exchange Reaction
}

\author{
Fabricio Vargas, * Fabiano T. Toledo and João V. Comasseto \\ Instituto de Química, Universidade de São Paulo, Av. Prof. Lineu Prestes, 748, \\ 05508-000 São Paulo-SP, Brazil
}

\section{Experimental}

\section{General}

Elemental tellurium was purchased from Sigma Aldrich. All reagents and solvents were purified and dried using procedures described in the literature. ${ }^{1}$ THF was distilled under nitrogen from sodium/benzophenone just before use. $N$-Butyllithium was titrated using 1,10-phenanthroline as indicator prior to use. Lithium-naphthalenide (LiNp) was prepared according to the procedure described in the literature. $^{2}$ All operations were carried out in flame-dried glassware. Column chromatographic separations were performed over Acros Organics silica gel (0.035-0.075 mm; pore diameter $c a .6 \mathrm{~nm}$ ). The melting points were determined using a Büchi, model B-545. Optical rotations were determined on a Perkin Elmer 343 polarimeter and IR spectra were recorded on a Bomem MB-100 spectrophotometer. NMR spectra were recorded on Varian-Inova $(300 \mathrm{MHz}$, ${ }^{1} \mathrm{H} ; 75 \mathrm{MHz},{ }^{13} \mathrm{C}$ ) or Bruker model DRX-500 (500 MHz, ${ }^{1} \mathrm{H} ; 125 \mathrm{MHz},{ }^{13} \mathrm{C}$ ) spectrometers using $\mathrm{CDCl}_{3}$ as solvent. The internal references were TMS ( ${ }^{1} \mathrm{H}$ NMR), the central peak of the $\mathrm{CDCl}_{3}$ signal $\left({ }^{13} \mathrm{C} \mathrm{NMR}\right)$ and a capillary of diphenyl ditelluride $1 \mathrm{~mol}^{-1}\left({ }^{125} \mathrm{Te} \mathrm{NMR}\right)$. High resolution mass spectroscopy was performed using a LC-MS - Bruker Daltonics instrument at the Microanalytical Laboratory of the Institute of Chemistry, University of São Paulo.

\section{General procedure for the preparation of tellurium amines}

n-Butyllithium (1 mmol, $1.5 \mathrm{~mol} \mathrm{~L}^{-1}$ in hexane) was slowly added at room temperature to a suspension of elemental tellurium $(1.2 \mathrm{mmol})$ in dry THF $(5 \mathrm{~mL})$. Deoxygenated ethanol $(2 \mathrm{~mL})$ was added to the light yellow solution of lithium butyl tellurolate so formed, and the resulting red-brown mixture was stirred at room

*e-mail: fvargas@iq.usp.br temperature for $10 \mathrm{~min}$ and subsequently cooled to $0{ }^{\circ} \mathrm{C}$. The corresponding aziridine or mesylate $(1 \mathrm{mmol})$ was added in a single portion, and the resulting mixture was stirred for $2 \mathrm{~h}$ at room temperature. The mixture was quenched with a saturated $\mathrm{NH}_{4} \mathrm{Cl}$ solution and extracted with $\mathrm{CH}_{2} \mathrm{Cl}_{2}$, and the combined organic fractions were dried over $\mathrm{MgSO}_{4}$, and filtered. The solvent was removed in vacuo, yielding the crude products, which were purified by flash chromatography.<smiles>[R16]C(=O)OCC[Te]CCCC</smiles>

tert-Butyl 2-(butyltellanyl)ethylcarbamate (1a)

The $N$-Boc $\beta$-telluro amine 1a was prepared according to the general procedure using $\mathrm{BocHNCH}_{2} \mathrm{CH}_{2} \mathrm{OMs}$ as starting material. Yield: $76 \%$; yellow oil; IR $v_{\text {max }}(\mathrm{film}) / \mathrm{cm}^{-1}$ :

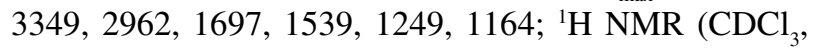
$500 \mathrm{MHz}) \delta 4.96$ (br s, 1H), 3.43-3.41 (m, 2H), $2.72(\mathrm{t}$, $J 7.1 \mathrm{~Hz}, 2 \mathrm{H}$ ), 2.66 (t, J 7.5 Hz, 2H), 1.72 (qui, J 7.5 Hz, 2H), 1.44 (s, 9H), $1.38(\mathrm{sex}, J 7.5 \mathrm{~Hz}, 2 \mathrm{H}), 0.91(\mathrm{t}, J 7.5 \mathrm{~Hz}$, $3 \mathrm{H}) ;{ }^{13} \mathrm{C} \mathrm{NMR}\left(\mathrm{CDCl}_{3}, 125 \mathrm{MHz}\right) \delta 155.6,79.1,42.4,34.2$, 28.4, 24.9, 13.3, 3.1, 2.7; ${ }^{125} \mathrm{Te} \mathrm{NMR}\left(\mathrm{CDCl}_{3}, 157 \mathrm{MHz}\right) \delta$ 182.6; HRMS-ESI $m / z$ calculated for $\mathrm{C}_{11} \mathrm{H}_{23} \mathrm{NO}_{2} \mathrm{Te}+\mathrm{Na}^{+}$ 354.0690, found 354.0703.<smiles>[R][Te][Te]CCNC(=O)O</smiles>

\section{Benzyl 2-(butyltellanyl)ethylcarbamate $(\mathbf{1 b})$}

The $N$-Cbz $\beta$-telluro amine $\mathbf{1 b}$ was prepared according to the general procedure using $\mathrm{CbzHNCH}_{2} \mathrm{CH}_{2} \mathrm{OMs}$ as starting material. Yield: 78\%; yellow oil; IR $v_{\max }($ film $) / \mathrm{cm}^{-1}$ : 3331, 2957, 1701, 1523, 1247; ${ }^{1} \mathrm{H} \mathrm{NMR}\left(\mathrm{CDCl}_{3}, 500 \mathrm{MHz}\right)$ $\delta$ 7.33-7.28 (m, 5H), 5.15 (br s, 1H), $5.10(\mathrm{~s}, 2 \mathrm{H}), 3.48$ (qua, $J 7.0 \mathrm{~Hz}, 2 \mathrm{H}), 2.73(\mathrm{t}, J 7.0 \mathrm{~Hz}, 2 \mathrm{H}), 2.63(\mathrm{t}, J 7.0 \mathrm{~Hz}, 2 \mathrm{H})$,

\footnotetext{
${ }^{1}$ Perrin, D. D.; Armarego, W. L. F.; Purification of Laboratory Chemicals, Pergamon: Oxford, 1980.

${ }^{2}$ Screttas, C. G.; Micha-Screttas, M.; J. Org. Chem. 1978, 43, 1064.
} 
1.68 (qui, $J 7.0 \mathrm{~Hz}, 2 \mathrm{H}), 1.37$ (sex, J 7.0 Hz, 2H), 0.90 (t, $J$ $7.0 \mathrm{~Hz}, 3 \mathrm{H}) ;{ }^{13} \mathrm{C} \mathrm{NMR}\left(\mathrm{CDCl}_{3}, 125 \mathrm{MHz}\right) \delta 156.0,136.3$, $128.3,127.9$ (2C), 66.5, 42.7, 34.1, 24.8, 13.2, 2.8, $2.7 ;{ }^{125} \mathrm{Te}$ $\mathrm{NMR}\left(\mathrm{CDCl}_{3}, 157 \mathrm{MHz}\right) \delta 183.5 ; \mathrm{HRMS}-\mathrm{ESI} \mathrm{m} / \mathrm{z}$ calculated for $\mathrm{C}_{14} \mathrm{H}_{21} \mathrm{NO}_{2} \mathrm{Te}+\mathrm{Na}^{+} 388.0532$, found 388.0532.

$$
\mathrm{TsHN} \Upsilon \mathrm{TeBu}
$$

$N-(2-(B u t y l t e l l a n y l) e t h y l)-4-m e t h y l b e n z e n e s u l f o n a m i d e$ (1c)

The $N$-Ts $\beta$-telluro amine 1c was prepared according to the general procedure using $N$-Ts aziridine as starting material. Yield: 83\%; yellow oil; IR $v_{\max }($ film $) / \mathrm{cm}^{-1}: 3277$, 2957, 2926, 2867, 1597, 1455, 1325, 1156; ${ }^{1} \mathrm{H}$ NMR $\left(\mathrm{CDCl}_{3}, 300 \mathrm{MHz}\right) \delta 7.80-7.73(\mathrm{~m}, 2 \mathrm{H}), 7.31-7.24(\mathrm{~m}$, 2H), $3.20(\mathrm{t}, J 7.2 \mathrm{~Hz}, 2 \mathrm{H}), 2.63(\mathrm{t}, J 7.2 \mathrm{~Hz}, 2 \mathrm{H}), 2.54$ (t, J 7.2 Hz, 2H), 2.42 (s, 3H), 1.83 (qui, J 7.2 Hz, 2H), 1.32 (sex, J 7.2 Hz, 2H), 0.88 (t, J 7.2 Hz, 3H); ${ }^{13} \mathrm{C}$ NMR $\left(\mathrm{CDCl}_{3}, 75 \mathrm{MHz}\right) \delta 143.5,137.1,129.8,127.1,44.8,34.2$, 25.0, 21.5, 13.4, 3.3, 2.3; ${ }^{125} \mathrm{Te} \mathrm{NMR}\left(\mathrm{CDCl}_{3}, 157 \mathrm{MHz}\right)$ $\delta$ 192.2; HRMS-ESI $m / z$ calculated for $\mathrm{C}_{13} \mathrm{H}_{21} \mathrm{NO}_{2} \mathrm{STe}+$ $\mathrm{Na}^{+}$408.0253, found 408.0250.<smiles>[R18]NCC[Te][Ba]</smiles>

\section{N-(2-(Butyltellanyl)ethyl)benzamide (1d)}

The $N$-Bz $\beta$-telluro amine 1d was prepared according to the general procedure using $N$-Bz aziridine as starting material. Yield: $82 \%$; yellow oil; IR $v_{\max }($ film $) / \mathrm{cm}^{-1}: 3308$, 2957, 1642, 1306; ${ }^{1} \mathrm{H} \mathrm{NMR}\left(\mathrm{CDCl}_{3}, 500 \mathrm{MHz}\right) \delta$ 7.79-7.78 (m, 2H), 7.50-7.47 (m, 1H), 7.43-7.39 (m, 2H), 6.80 (br s, $1 \mathrm{H}), 3.74-3.70(\mathrm{~m}, 2 \mathrm{H}), 2.85(\mathrm{t}, J 7.0 \mathrm{~Hz}, 2 \mathrm{H}), 2.67(\mathrm{t}, J 7.0$ $\mathrm{Hz}, 2 \mathrm{H}$ ), 1.72 (qui, J 7.5 Hz, 2H), 1.36 (sex, J 7.5 Hz, 2H), $0.89(\mathrm{t}, J 7.0 \mathrm{~Hz}, 3 \mathrm{H}) ;{ }^{13} \mathrm{C} \mathrm{NMR}\left(\mathrm{CDCl}_{3}, 125 \mathrm{MHz}\right) \delta 167.2$, 134.4, 131.5, 128.5, 126.9, 41.3, 34.2, 25.0, 13.3, 3.0, 2.6; ${ }^{125} \mathrm{Te} \mathrm{NMR}\left(\mathrm{CDCl}_{3}, 157 \mathrm{MHz}\right) \delta$ 184.6; HRMS-ESI $\mathrm{m} / \mathrm{z}$ calculated for $\mathrm{C}_{13} \mathrm{H}_{19} \mathrm{NOTe}+\mathrm{Na}^{+} 358.0427$, found 358.0407.

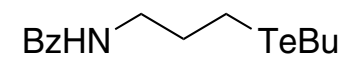

$N-(3-(B u t y l t e l l a n y l) p r o p y l)$ benzamide (1e)

The $N$-Bz $\gamma$-telluro amine $1 \mathrm{e}$ was prepared according to the general procedure using $\mathrm{N}-\mathrm{BzNHCH}_{2} \mathrm{CH}_{2} \mathrm{CH}_{2} \mathrm{OMs}$ as starting material. Yield: $75 \%$; yellow oil; IR $v_{\max }$ (film) $/ \mathrm{cm}^{-1}$ : 3326, 2955, 1650, 1308; ${ }^{1} \mathrm{H} \mathrm{NMR}\left(\mathrm{CDCl}_{3}, 500 \mathrm{MHz}\right) \delta$ 7.79-7.77 (m, 2H), 7.47-7.44 (m, 1H), 7.39-7.36 (m, 2H), 6.99 (br s, 1H), 3.47 (qua, J 7.0 Hz, 2H), 2.62 (t, J 7.0 Hz, 4H), 2.04 (qui, J7.0 Hz, 2H), 1.70 (qui, J 7.5 Hz, 2H), 1.36 (sex, J7.5 Hz, 2H), 0.89 (t, J7.0 Hz, 3H); ${ }^{13} \mathrm{C} \mathrm{NMR}\left(\mathrm{CDCl}_{3}\right.$, $125 \mathrm{MHz}) \delta 167.8,134.6,131.6,128.5,127.0,42.0,34.3$, $32.0,25.1,13.5,3.0,-1.3 ;{ }^{125} \mathrm{Te} \mathrm{NMR}\left(\mathrm{CDCl}_{3}, 157 \mathrm{MHz}\right)$ $\delta$ 232.7; HRMS-ESI $m / z$ calculated for $\mathrm{C}_{14} \mathrm{H}_{21} \mathrm{NOTe}+\mathrm{Na}^{+}$ 372.0583 , found 372.0577 .<smiles>[R16]NCCCC[Te]C(=O)c1ccccc1</smiles>

$N$-(4-(Butyltellanyl)butyl)benzamide (1f)

The $N$-Bz $\delta$-telluro amine $\mathbf{1 f}$ was prepared according to the general procedure using $\mathrm{N}-\mathrm{BzNHCH}_{2}\left(\mathrm{CH}_{2}\right)_{2} \mathrm{CH}_{2} \mathrm{OMs}$ as starting material. Yield: $80 \%$; yellow oil; IR $v_{\text {max }}($ film $) / \mathrm{cm}^{-1}$ : 3318, 2956, 1643, 1308; ${ }^{1} \mathrm{H} \mathrm{NMR}\left(\mathrm{CDCl}_{3}, 500 \mathrm{MHz}\right) \delta$ 7.77-7.76 (m, 2H), 7.50-7.46 (m, 1H), 7.42-7.39 (m, 2H), 6.41 (br s, 1H), 3.46 (qua, J 7.0 Hz, 2H), 2.66-2.62 (m, 4H), 1.82 (qui, $J 7.5 \mathrm{~Hz}, 2 \mathrm{H}$ ), 1.74-1.67 (m, 4H), 1.36 (sex, $J$ $7.5 \mathrm{~Hz}, 2 \mathrm{H}), 0.90$ (t, J 7.5 Hz, 3H); ${ }^{13} \mathrm{C} \mathrm{NMR}\left(\mathrm{CDCl}_{3}, 125\right.$ $\mathrm{MHz}) \delta 167.5,134.6,131.3,128.4,126.8,39.3,34.2,31.9$, 29.5, 25.0, 13.3 , 2.7, 1.8; ${ }^{125} \mathrm{Te} \mathrm{NMR}\left(\mathrm{CDCl}_{3}, 157 \mathrm{MHz}\right) \delta$ 225.4; HRMS-ESI $m / z$ calculated for $\mathrm{C}_{15} \mathrm{H}_{23} \mathrm{NOTe}+\mathrm{Na}^{+}$ 386.0740 , found 386.0737 .

General procedure for the tellurium/lithium exchange reaction

$n$-Butyllithium (2 mmol, $1.5 \mathrm{~mol} \mathrm{~L}^{-1}$ in hexane) was slowly added to a solution of telluro amine $(1 \mathrm{mmol})$ in dry THF $(12 \mathrm{~mL})$ at $-78{ }^{\circ} \mathrm{C}$. The progress of the tellurium/ lithium exchange reaction was monitorated by TLC. Then, a solution of lithium naphthalenide (LiNp) (2.5 mmol) in THF was added dropwise to the mixture and stirred at $-78{ }^{\circ} \mathrm{C}$ for $2 \mathrm{~h}$. To the resulting mixture was added a solution of the corresponding electrophile $(2 \mathrm{mmol})$ in THF $(1 \mathrm{~mL})$ and then allowed to rise to room temperature overnight. The mixture was quenched with a saturated $\mathrm{NH}_{4} \mathrm{Cl}$ solution and extracted with $\mathrm{CH}_{2} \mathrm{Cl}_{2}$, and the combined organic fractions were collected, dried over $\mathrm{MgSO}_{4}$, and filtered. The solvent was removed in vacuum, yielding the crude products, which were purified by flash chromatography.<smiles>O=C(O)NCCC(O)c1ccccc1</smiles>

N-(3-Hydroxy-3-phenylpropyl)benzamide (2a)

Yield: 79\%; colorless oil; IR $v_{\max }($ film $) / \mathrm{cm}^{-1}: 3343$, 1640, 1541, 1310; ${ }^{1} \mathrm{H}$ NMR $\left(\mathrm{CDCl}_{3}, 500 \mathrm{MHz}\right) \delta 7.75-7.72$ (m, 2H), 7.49-7.46 (m, 1H), 7.43-7.37 (m, 2H), 7.35-7.33 (m, 4H), 7.27-7.23 (m, 1H), 7.05 (br s, 1H), 4.80 (dd, J 8.5 $\mathrm{Hz}, J 4.0 \mathrm{~Hz}, 1 \mathrm{H}), 3.84-3.77(\mathrm{~m}, 1 \mathrm{H}), 3.45-3.41(\mathrm{~m}, 1 \mathrm{H})$, 1.99-1.93 (m, 2H); ${ }^{13} \mathrm{C} \mathrm{NMR}\left(\mathrm{CDCl}_{3}, 125 \mathrm{MHz}\right) \delta 168.2$, 144.1, 134.2, 131.4, 128.5, 128.4, 127.4, 126.9, 125.6, 72.5, $38.4,37.5$; HRMS-ESI $m / z$ calculated for $\mathrm{C}_{16} \mathrm{H}_{17} \mathrm{NO}_{2}+\mathrm{Na}^{+}$ 278.1157 , found 278.1152 . 
<smiles>Cc1ccc(C(O)CCNC(=O)c2ccccc2)cc1</smiles>

N-(3-Hydroxy-3-p-tolylpropyl)benzamide (2b)

Yield: $72 \%$; orange solid; $\mathrm{mp} 89.6-91.2{ }^{\circ} \mathrm{C}$; IR $v_{\max }(\mathrm{KBr}) / \mathrm{cm}^{-1}: 3359,1639,1551,1075,927 ;{ }^{1} \mathrm{H}$ NMR $\left(\mathrm{CDCl}_{3}, 500 \mathrm{MHz}\right) \delta$ 7.74-7.72 (m, 2H), 7.50-7.46 (m, $1 \mathrm{H}), 7.42-7.39(\mathrm{~m}, 2 \mathrm{H}), 7.24(\mathrm{~d}, J 8.0 \mathrm{~Hz}, 2 \mathrm{H}), 7.14(\mathrm{~d}, J$ $8.0 \mathrm{~Hz}, 2 \mathrm{H}), 6.90$ (br s, 1H), 4.79 (t, J $6.5 \mathrm{~Hz}, 1 \mathrm{H}), 3.85-$ $3.78(\mathrm{~m}, 1 \mathrm{H}), 3.48-3.42(\mathrm{~m}, 1 \mathrm{H}), 2.32$ (s, 3H), 1.98 (qua, $J$ $6.0 \mathrm{~Hz}, 2 \mathrm{H}) ;{ }^{13} \mathrm{C} \mathrm{NMR}\left(\mathrm{CDCl}_{3}, 125 \mathrm{MHz}\right) \delta 167.9,141.1$, 137.0, 134.2, 131.3, 129.0, 128.4, 126.8, 125.5, 72.5, 38.3, 37.5, 21.0; HRMS-ESI $m / z$ calculated for $\mathrm{C}_{17} \mathrm{H}_{19} \mathrm{NO}_{2}+\mathrm{Na}^{+}$ 292.1313, found 292.1314 .<smiles>Cc1ccccc1C(O)CCNC(=O)c1ccccc1</smiles>

$\mathrm{N}$-(3-Hydroxy-3-o-tolylpropyl)benzamide (2c)

Yield: 82\%; white solid; mp 104-106 ${ }^{\circ} \mathrm{C}$; IR $v_{\max }(\mathrm{KBr}) / \mathrm{cm}^{-1} 3309,1628,1558,1050 ;{ }^{1} \mathrm{H} \mathrm{NMR}\left(\mathrm{CDCl}_{3}\right.$, $500 \mathrm{MHz}) \delta$ 7.79-7.77 (m, 2H), 7.56-7.51 (m, 2H), 7.47$7.44(\mathrm{~m}, 2 \mathrm{H}), 7.27-7.24(\mathrm{~m}, 1 \mathrm{H}), 7.21-7.18(\mathrm{~m}, 1 \mathrm{H}), 7.16$ - 7.14 (m, 1H), 6.95 (br s, 1H), 5.11 (dd, J $8.8 \mathrm{~Hz}, J 3.3$ $\mathrm{Hz}, 1 \mathrm{H}), 3.94-3.91(\mathrm{~m}, 1 \mathrm{H}), 3.53-3.47(\mathrm{~m}, 1 \mathrm{H}), 2.33$ (s, $3 \mathrm{H}), 2.02-1.95(\mathrm{~m}, 2 \mathrm{H}) ;{ }^{13} \mathrm{C}$ NMR $\left(\mathrm{CDCl}_{3}, 125 \mathrm{MHz}\right) \delta$ $168.1,142.1,134.3,133.9,131.4,130.4,128.5,127.2$, 126.9, 126.3, 125.0, 69.4, 37.8, 36.9, 18.9; HRMS-ESI $\mathrm{m} / \mathrm{z}$ calculated for $\mathrm{C}_{17} \mathrm{H}_{19} \mathrm{NO}_{2}+\mathrm{Na}^{+} 292.1313$, found 292.1301.<smiles>Cc1cccc(C(O)CCNC(=O)c2ccccc2)c1</smiles>

$N$-(3-hydroxy-3-m-tolylpropyl)benzamide (2d)

Yield: $72 \%$; colorless oil; IR $v_{\max }\left(\right.$ film) $/ \mathrm{cm}^{-1}: 3354$, $1644,1538,1075 ;{ }^{1} \mathrm{H} \mathrm{NMR}\left(\mathrm{CDCl}_{3}, 500 \mathrm{MHz}\right) \delta 7.74-7.72$ $(\mathrm{m}, 2 \mathrm{H}), 7.49-7.46(\mathrm{~m}, 1 \mathrm{H}), 7.40-7.37(\mathrm{~m}, 2 \mathrm{H}), 7.22-7.19$ (m, 1H), 7.16-7.12 (m, 2H), 7.07 - $7.06(\mathrm{~m}, 1 \mathrm{H}), 7.03$ (br s, 1H), $4.77(\mathrm{dd}, J 8.0 \mathrm{~Hz}, J 4.5 \mathrm{~Hz}, 1 \mathrm{H}), 3.83-3.77(\mathrm{~m}$, $1 \mathrm{H}), 3.46-3.43(\mathrm{~m}, 1 \mathrm{H}), 2.32(\mathrm{~s}, 3 \mathrm{H}), 1.98-1.95(\mathrm{~m}, 2 \mathrm{H})$; ${ }^{13} \mathrm{C} \mathrm{NMR}\left(\mathrm{CDCl}_{3}, 125 \mathrm{MHz}\right) \delta 168.2,144.1,137.9,134.0$, 131.3, 128.3, 128.2, 128.0, 126.9, 126.3, 122.6, 72.3, 38.2, 37.5, 21.3; HRMS-ESI $m / z$ calculated for $\mathrm{C}_{17} \mathrm{H}_{19} \mathrm{NO}_{2}+\mathrm{Na}^{+}$ 292.1313, found 292.1309.<smiles>CCOc1ccc(C(O)CCNC(=O)c2ccccc2)cc1</smiles>

$N$-(3-(4-Ethoxyphenyl)-3-hydroxypropyl)benzamide (2e)

Yield: 70\%; yellow oil; IR $v_{\text {max }}$ (film) $/ \mathrm{cm}^{-1}: 3341,1642$, $1541,1512,1304,1245,1047 ;{ }^{1} \mathrm{H}$ NMR $\left(\mathrm{CDCl}_{3}, 500 \mathrm{MHz}\right)$ d 7.75-7.73 (m, 2H), 7.50-7.46 (m, 1H), 7.42-7.39 (m, 2H), 7.28-7.25 (m, 2H), $6.91(\mathrm{br} \mathrm{s}, 1 \mathrm{H}), 6.87-6.85(\mathrm{~m}, 2 \mathrm{H}), 4.77$ (t, $J 6.5 \mathrm{~Hz}, 1 \mathrm{H}$ ), 4.00 (qua, $J 7.0 \mathrm{~Hz}, 2 \mathrm{H}$ ), 3.85-3.78 (m, $1 \mathrm{H}), 3.47-3.41(\mathrm{~m}, 1 \mathrm{H}), 1.97$ (qua, $J 6.5 \mathrm{~Hz}, 2 \mathrm{H}), 1.40(\mathrm{t}, J$ $7.0 \mathrm{~Hz}, 3 \mathrm{H}) ;{ }^{13} \mathrm{C} \mathrm{NMR}\left(\mathrm{CDCl}_{3}, 125 \mathrm{MHz}\right) \delta 167.9,158.1$, 136.1, 134.1, 131.2, 128.3, 126.8, 126.7, 114.2, 72.1, 63.3, 38.1, 37.4, 14.7; HRMS-ESI $\mathrm{m} / \mathrm{z}$ calculated for $\mathrm{C}_{18} \mathrm{H}_{21} \mathrm{NO}_{3}$ $+\mathrm{Na}^{+} 322.1419$, found 322.1411 .<smiles>O=C(O)NCCC(O)c1ccco1</smiles>

$N$-(3-(Furan-2-yl)-3-hydroxypropyl)benzamide (2f)

Yield: 68\%; orange oil; IR $v_{\text {max }}($ film $) / \mathrm{cm}^{-1}: 3335,1640$, $1544,1310,1148 ;{ }^{1} \mathrm{H} \mathrm{NMR}\left(\mathrm{CDCl}_{3}, 500 \mathrm{MHz}\right) \delta 7.75-7.72$ (m, 2H), 7.48-7.45 (td, J 7.0 Hz, J 1.5 Hz, 1H), 7.40-7.36 (m, 2H), $7.32(\mathrm{~s}, 1 \mathrm{H}), 7.07$ (br s, 1H), 6.30-6.28 (m, 1H), 6.24-6.23 (m, 1H), $4.81(\mathrm{t}, J 7.0 \mathrm{~Hz}, 1 \mathrm{H}), 3.83-3.80(\mathrm{~m}, 1 \mathrm{H})$, 3.50-3.44 (m, $1 \mathrm{H}), 2.12-2.03(\mathrm{~m}, 2 \mathrm{H}) ;{ }^{13} \mathrm{C} \mathrm{NMR}\left(\mathrm{CDCl}_{3}\right.$, $125 \mathrm{MHz}) \delta 168.4,156.2,141.7,134.0,131.4,128.5,126.9$, 110.1, 105.7, 65.6, 36.9, 34.8; HRMS-ESI $\mathrm{m} / z$ calculated for $\mathrm{C}_{14} \mathrm{H}_{15} \mathrm{NO}_{3}+\mathrm{Na}^{+}$268.0950, found 268.0948.<smiles>CCCCCCCC(O)CCNC(=O)c1ccccc1</smiles>

$N$-(3-Hydroxydecyl)benzamide (2g)

Yield: 79\%; colorless oil; IR $v_{\max }($ film $) / \mathrm{cm}^{-1}: 3305$, $2922,1634,1549 ;{ }^{1} \mathrm{H}$ NMR $\left(\mathrm{CDCl}_{3}, 500 \mathrm{MHz}\right) \delta$ 7.78-7.76 (m, 2H), 7.49-7.46 (m, 1H), 7.43-7.39 (m, 2H), 7.05 (br $\mathrm{s}, 1 \mathrm{H}), 3.86-3.83(\mathrm{~m}, 1 \mathrm{H}), 3.72-3.68(\mathrm{~m}, 1 \mathrm{H}), 3.39-3.34$ $(\mathrm{m}, 1 \mathrm{H}), 1.77-1.74(\mathrm{~m}, 1 \mathrm{H}), 1.62-1.58(\mathrm{~m}, 1 \mathrm{H}), 1.52-1.41$ (m, 3H), 1.27-1.23 (m, 9H), $0.87(\mathrm{t}, J 7.0 \mathrm{~Hz}, 3 \mathrm{H}) ;{ }^{13} \mathrm{C}$ NMR $\left(\mathrm{CDCl}_{3}, 125 \mathrm{MHz}\right) \delta 168.2,134.2,131.4,128.5$, 127.0, 69.9, 37.5, 36.5, 31.8, 29.6, 29.2, 25.8, 22.6, 14.1; HRMS-ESI $m / z$ calculated for $\mathrm{C}_{17} \mathrm{H}_{27} \mathrm{NO}_{2}+\mathrm{Na}^{+} 300.1939$, found 300.1949 .<smiles>CCCC(O)CCN[18O]</smiles>

N-(3-Hydroxyhexyl)benzamide (2h)

Yield: 75\%; yellow oil; IR $v_{\text {max }}($ film $) / \mathrm{cm}^{-1}: 3334,2957$, 
1642,$1545 ;{ }^{1} \mathrm{H} \mathrm{NMR}\left(\mathrm{CDCl}_{3}, 500 \mathrm{MHz}\right) \delta$ 7.78-7.76 (m, $2 \mathrm{H}), 7.49-7.46(\mathrm{~m}, 1 \mathrm{H}), 7.42-7.39(\mathrm{~m}, 2 \mathrm{H}), 7.05$ (br s, $1 \mathrm{H}), 3.87-3.85(\mathrm{~m}, 1 \mathrm{H}), 3.73-3.70(\mathrm{~m}, 1 \mathrm{H}), 3.39-3.34(\mathrm{~m}$, $1 \mathrm{H}), 1.76-1.73(\mathrm{~m}, 1 \mathrm{H}), 1.62-1.58(\mathrm{~m}, 1 \mathrm{H}), 1.52-1.42(\mathrm{~m}$, $4 \mathrm{H}), 0.91(\mathrm{t}, J 7.0 \mathrm{~Hz}, 3 \mathrm{H}) ;{ }^{13} \mathrm{C} \mathrm{NMR}\left(\mathrm{CDCl}_{3}, 125 \mathrm{MHz}\right)$ $\delta 168.4,134.2,131.4,128.4,127.0,69.4,39.5,37.4,36.4$, 18.9, 14.0; HRMS-ESI $m / z$ calculated for $\mathrm{C}_{13} \mathrm{H}_{19} \mathrm{NO}_{2}+\mathrm{Na}^{+}$ 244.1313, found 244.1307 .<smiles>CC(C)(C)C(O)CCNC(=O)c1ccccc1</smiles>

N-(3-Hydroxy-4,4-dimethylpentyl)benzamide (2i)

Yield: 76\%; white solid; mp 143-144 ${ }^{\circ} \mathrm{C}$; IR $v_{\max }(\mathrm{KBr}) / \mathrm{cm}^{-1}: 3329,2968,1637,1549 ;{ }^{1} \mathrm{H} \mathrm{NMR}\left(\mathrm{CDCl}_{3}\right.$, $500 \mathrm{MHz}) \delta$ 7.78-7.77 (m, 2H), 7.49-7.48 (m, 1H), 7.44$7.41(\mathrm{~m}, 1 \mathrm{H}), 6.91(\mathrm{br} \mathrm{s}, 1 \mathrm{H}), 3.96-3.93(\mathrm{~m}, 1 \mathrm{H}), 3.37-3.33$ $(\mathrm{m}, 2 \mathrm{H}), 1.85-1.81(\mathrm{~m}, 1 \mathrm{H}), 1.57-1.55(\mathrm{~m}, 1 \mathrm{H}), 0.92(\mathrm{~s}$, $9 \mathrm{H}) ;{ }^{13} \mathrm{C}$ NMR $\left(\mathrm{CDCl}_{3}, 125 \mathrm{MHz}\right) \delta 168.0,134.4,131.4$, 128.5, 126.9, 78.3, 38.4, 34.8, 30.8, 25.7; HRMS-ESI $\mathrm{m} / \mathrm{z}$ calculated for $\mathrm{C}_{14} \mathrm{H}_{21} \mathrm{NO}_{2}+\mathrm{Na}^{+} 258.1470$, found 258.1456 .<smiles>CC(O)(CCNC(=O)c1ccccc1)c1ccccc1</smiles>

$N$-(3-Hydroxy-3-phenylbutyl)benzamide (2j)

Yield: 69\%; yellow oil; IR $v_{\text {max }}($ film $) / \mathrm{cm}^{-1}: 3344,1642$, $1540,1489,1445,1312 ;{ }^{1} \mathrm{H}$ NMR $\left(\mathrm{CDCl}_{3}, 500 \mathrm{MHz}\right) \delta$ 7.63-7.61 (m, 2H), 7.47-7.42 (m, 3H), 7.37-7.30 (m, 4H), 7.23-7.20 (m, 1H), $6.93($ br s, $1 \mathrm{H}), 3.59-3.52(\mathrm{~m}, 1 \mathrm{H})$, 3.35-3.29 (m, 1H), $2.87(\mathrm{~s}, 1 \mathrm{H}), 2.19-2.13(\mathrm{~m}, 1 \mathrm{H}), 2.09-$ $2.03(\mathrm{~m}, 1 \mathrm{H}), 1.62(\mathrm{~s}, 3 \mathrm{H}) ;{ }^{13} \mathrm{C} \mathrm{NMR}\left(\mathrm{CDCl}_{3}, 125 \mathrm{MHz}\right)$ $\delta 167.3,147.2,134.4,131.2,128.5,128.3,126.8,126.7$, 124.6, 74.9, 42.3, 36.3, 31.1. HRMS-ESI $\mathrm{m} / \mathrm{z}$ calculated for $\mathrm{C}_{17} \mathrm{H}_{19} \mathrm{NO}_{2}+\mathrm{Na}^{+} 292.1313$, found 292.1302 .

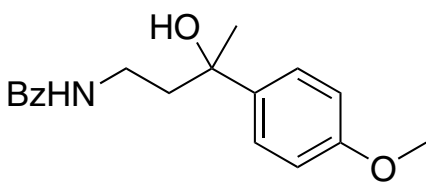

$\mathrm{N}$-(3-Hydroxy-3-(4-methoxyphenyl)butyl)benzamide (2k)

Yield: $65 \%$; orange oil; IR $v_{\max }($ film $) / \mathrm{cm}^{-1}: 3355,1643$, 1593, 1511, 1299, 1248, 1179; ${ }^{1} \mathrm{H} \mathrm{NMR}\left(\mathrm{CDCl}_{3}, 500 \mathrm{MHz}\right)$ $\delta$ 7.64-7.61 (m, 2H), 7.46-7.43 (m, 1H), 7.38-7.34 (m, 4H), 6.89 (br s, $1 \mathrm{H}), 6.86-6.83(\mathrm{~m}, 2 \mathrm{H}), 3.76(\mathrm{~s}, 3 \mathrm{H}), 3.58-3.53$ (m, 1H), 3.36-3.32 (m, 1H), 2.74 (br s, 1H), 2.15-2.10 (m, $1 \mathrm{H}), 2.07-2.01(\mathrm{~m}, 1 \mathrm{H}), 1.60(\mathrm{~s}, 3 \mathrm{H}) ;{ }^{13} \mathrm{C} \mathrm{NMR}\left(\mathrm{CDCl}_{3}, 125\right.$ MHz) $\delta 167.2,158.1,139.4,134.2,131.1,128.2,126.7$, 125.7, 113.5, 74.3, 55.1, 42.1, 36.3, 30.9; HRMS-ESI $\mathrm{m} / \mathrm{z}$ calculated for $\mathrm{C}_{18} \mathrm{H}_{21} \mathrm{NO}_{3}+\mathrm{Na}^{+} 322.1419$, found 322.1420 .<smiles>O=C(NCCC(O)(c1ccccc1)c1ccccc1)c1ccccc1</smiles>

$N$-(3-Hydroxy-3,3-diphenylpropyl)benzamide (2l)

Yield: $61 \%$; white solid; mp $149-150{ }^{\circ} \mathrm{C}$; IR $v_{\max }(\mathrm{KBr}) / \mathrm{cm}^{-1}: 3435,3331,1650,1532,1219 ;{ }^{1} \mathrm{H}$ NMR $\left(\mathrm{CDCl}_{3}, 500 \mathrm{MHz}\right) \delta 7.62-7.61(\mathrm{~m}, 2 \mathrm{H}), 7.49-7.45(\mathrm{~m}$, $5 \mathrm{H}), 7.40-7.37(\mathrm{~m}, 2 \mathrm{H}), 7.34-7.31(\mathrm{~m}, 4 \mathrm{H}), 7.25-7.22(\mathrm{~m}$, 2H), 6.74 (br s, 1H), 3.55 (qua, $J 6.0 \mathrm{~Hz}, 2 \mathrm{H}), 2.65(\mathrm{t}, J$ $6.0 \mathrm{~Hz}, 2 \mathrm{H}) ;{ }^{13} \mathrm{C} \mathrm{NMR}\left(\mathrm{CDCl}_{3}, 125 \mathrm{MHz}\right) \delta 167.4,146.6$, 134.3, 131.2, 128.3, 127.0, 126.8, 125.9, 78.2, 40.7, 36.1; HRMS-ESI $m / z$ calculated for $\mathrm{C}_{22} \mathrm{H}_{21} \mathrm{NO}_{2}+\mathrm{Na}^{+} 354.1470$, found 354.1458 .<smiles>[R18]NCC[Si](C)(C)C</smiles>

$N$-(2-(Trimethylsilyl)ethyl)benzamide (2m)

Yield: 67\%; yellow oil; IR $v_{\text {max }}\left(\right.$ film) $/ \mathrm{cm}^{-1}: 3316,2953$, $1638,1543,1249$; ${ }^{1} \mathrm{H}$ NMR $\left(\mathrm{CDCl}_{3}, 500 \mathrm{MHz}\right) \delta 7.75$ $7.73(\mathrm{~m}, 2 \mathrm{H}), 7.49-7.46(\mathrm{~m}, 1 \mathrm{H}), 7.42-7.40(\mathrm{~m}, 2 \mathrm{H}), 6.12$ (br s, 1H), 3.52-3.47 (m, 2H), 0.93-0.90 (m, 2H), $0.06(\mathrm{~s}$, $9 \mathrm{H}) ;{ }^{13} \mathrm{C}$ NMR $\left(\mathrm{CDCl}_{3}, 125 \mathrm{MHz}\right) \delta 167.2,134.8,131.2$, 128.5, 126.7, 36.5, 17.7, -1.6; HRMS-ESI $\mathrm{m} / z$ calculated for $\mathrm{C}_{12} \mathrm{H}_{19} \mathrm{NOSi}+\mathrm{Na}^{+} 244.1134$, found 244.1136.

\section{$\mathrm{BzHN} \widehat{\Upsilon}$}

$N$-Ethylbenzamide (2n)

Yield: $78 \%$; yellow solid; $\mathrm{mp}$ 65.2-65.6 ${ }^{\circ} \mathrm{C}$; IR $v_{\max }(\mathrm{KBr}) / \mathrm{cm}^{-1}: 1637,1549,1310,1145 ;{ }^{1} \mathrm{H} \mathrm{NMR}\left(\mathrm{CDCl}_{3}\right.$, $500 \mathrm{MHz}) \delta$ 7.77-7.75 (m, 2H), 7.50-7.46 (m, 1H), 7.43$7.40(\mathrm{~m}, 2 \mathrm{H}), 6.18$ (br s, $1 \mathrm{H}$ ), 3.49 (qui, $J 7.0 \mathrm{~Hz}, 2 \mathrm{H}$ ), 1.25 $(\mathrm{t}, J 7.0 \mathrm{~Hz}, 3 \mathrm{H}) ;{ }^{13} \mathrm{C}$ NMR $\left(\mathrm{CDCl}_{3}, 125 \mathrm{MHz}\right) \delta 167.4$, 134.7, 131.1, 128.3, 126.8, 34.8, 14.7; HRMS-ESI $\mathrm{m} / \mathrm{z}$ calculated for $\mathrm{C}_{9} \mathrm{H}_{11} \mathrm{NO}+\mathrm{Na}^{+} 172.0738$, found 172.0731 .<smiles>O=C(NCCCC(O)c1ccccc1)c1ccccc1</smiles>

N-(4-Hydroxy-4-phenylbutyl)benzamide (2o)

Yield: $72 \%$; white solid; mp 75-76 ${ }^{\circ} \mathrm{C}$; IR $v_{\max }(\mathrm{KBr}) / \mathrm{cm}^{-1}: 3327,1638,1310,700 ;{ }^{1} \mathrm{H} \mathrm{NMR}\left(\mathrm{CDCl}_{3}\right.$, $500 \mathrm{MHz}) \delta$ 7.72-7.70 (m, 2H), 7.45-7.42 (m, 1H), 7.36$7.33(\mathrm{~m}, 2 \mathrm{H}), 7.30-7.29(\mathrm{~m}, 4 \mathrm{H}), 7.25-7.21(\mathrm{~m}, 1 \mathrm{H}), 6.79$ (br s, 1H), $4.68(\mathrm{dd}, J 7.5 \mathrm{~Hz}, J 5.0 \mathrm{~Hz}, 1 \mathrm{H}), 3.47-3.35$ $(\mathrm{m}, 2 \mathrm{H}), 1.85-1.52(\mathrm{~m}, 4 \mathrm{H}) ;{ }^{13} \mathrm{C} \mathrm{NMR}\left(\mathrm{CDCl}_{3}, 125 \mathrm{MHz}\right)$ $\delta$ 167.7, 144.6, 134.5, 131.2, 128.4, 128.3, 127.4, 126.9, 125.7, 73.9, 39.8, 36.1, 25.8; HRMS-ESI $\mathrm{m} / \mathrm{z}$ calculated for $\mathrm{C}_{17} \mathrm{H}_{19} \mathrm{NO}_{2}+\mathrm{Na}^{+} 292.1313$, found 292.1314. 
<smiles>O=C(NCCCCC(O)c1ccccc1)c1ccccc1</smiles>

$N$-(5-Hidroxy-5-phenylpentyl)benzamide (2p)

Yield: $70 \%$; yellow oil; IR $v_{\max }\left(\right.$ film) $/ \mathrm{cm}^{-1}: 3357,1643$, 1310,$701 ;{ }^{1} \mathrm{H}$ NMR $\left(\mathrm{CDCl}_{3}, 500 \mathrm{MHz}\right) \delta 7.70-7.68(\mathrm{~m}$, $2 \mathrm{H}), 7.44-7.41(\mathrm{~m}, 1 \mathrm{H}), 7.36-7.33(\mathrm{~m}, 2 \mathrm{H}), 7.28-7.27(\mathrm{~m}$, $4 \mathrm{H}), 7.24-7.19(\mathrm{~m}, 1 \mathrm{H}), 6.49(\mathrm{br} \mathrm{s}, 1 \mathrm{H}), 4.62(\mathrm{dd}, J 8.0 \mathrm{~Hz}$, $J 5.5 \mathrm{~Hz}, 1 \mathrm{H}$ ), 3.36 (qua, J 7.0 Hz, 2H), 1.81-1.75 (m, 1H), $1.72-1.65(\mathrm{~m}, 1 \mathrm{H}), 1.62-1.52(\mathrm{~m}, 2 \mathrm{H}), 1.51-1.42(\mathrm{~m}, 1 \mathrm{H})$, $1.38-1.29(\mathrm{~m}, 1 \mathrm{H}) ;{ }^{13} \mathrm{C} \mathrm{NMR}\left(\mathrm{CDCl}_{3}, 125 \mathrm{MHz}\right) \delta 167.7$, 144.7, 134.6, 131.2, 128.4, 128.3, 127.3, 126.8, 125.7, 74.1, 39.7, 38.4, 29.2, 23.0; HRMS-ESI $\mathrm{m} / \mathrm{z}$ calculated for $\mathrm{C}_{18} \mathrm{H}_{21} \mathrm{NO}_{2}+\mathrm{Na}^{+} 306.1470$, found 306.1461.<smiles>O=C(NCCc1ccccc1)c1ccccc1</smiles>

\section{$N$-Phenethylbenzamide (3a)}

Yield: $65 \%$; yellowish solid; mp $113-114{ }^{\circ} \mathrm{C}$; IR $v_{\max }(\mathrm{KBr}) / \mathrm{cm}^{-1}: 3344,1639,1544,1312,1193,695$; ${ }^{1} \mathrm{H} \mathrm{NMR}\left(\mathrm{CDCl}_{3}, 500 \mathrm{MHz}\right) \delta$ 7.69-7.67 (m, 2H), 7.49-7.46 (m, 1H), 7.41-7.38 (m, 2H), 7.34-7.31 (m, 2H), 7.26-7.23 (m, 3H), 6.17 (br s, 1H), 3.72 (qua, $J 7,0 \mathrm{~Hz}, 2 \mathrm{H}$ ), 2.94 (t, $J$ $7 ; 0 \mathrm{~Hz}, 2 \mathrm{H}) ;{ }^{13} \mathrm{C} \mathrm{NMR}\left(\mathrm{CDCl}_{3}, 125 \mathrm{MHz}\right) \delta 167.5,138.7$, 134.6, 131.3, 128.7, 128.6, 128.5, 126.8, 126.5, 41.1, 35.6; HRMS-ESI $m / z$ calculated for $\mathrm{C}_{15} \mathrm{H}_{15} \mathrm{NO}+\mathrm{Na}^{+} 248.1051$, found 248.1052 .<smiles>COc1ccc(CCNC(=O)c2ccccc2)cc1</smiles>

$N$-(4-Methoxyphenethyl)benzamide (3b)

Yield: 69\%; yellowish solid; mp 123-124 ${ }^{\circ} \mathrm{C}$; IR $v_{\max }(\mathrm{KBr}) / \mathrm{cm}^{-1}: 3320,1635,1538,1308,1243,693$; ${ }^{1} \mathrm{H} \mathrm{NMR}\left(\mathrm{CDCl}_{3}, 500 \mathrm{MHz}\right) \delta$ 7.70-7.68 (m, 2H), 7.50-7.46 $(\mathrm{m}, 1 \mathrm{H}), 7.42-7.39(\mathrm{~m}, 2 \mathrm{H}), 7.17-7.14(\mathrm{~m}, 2 \mathrm{H}), 6.88-6,86$ (m, 2H), 6.12 (br s, 1H), 3.80 (s, 1H), 3.69 (qua, $J 7.0 \mathrm{~Hz}$, $2 \mathrm{H}), 2.88(\mathrm{t}, J 7.0 \mathrm{~Hz}, 2 \mathrm{H}) .{ }^{13} \mathrm{C} \mathrm{NMR}\left(\mathrm{CDCl}_{3}, 125 \mathrm{MHz}\right)$ $\delta 167.5,158.3,134.7,131.4,130.9,129.8,128.5,126.8$, $114.1 ; 55.3,41.3,34.8$; HRMS-ESI $m / z$ calculated for $\mathrm{C}_{16} \mathrm{H}_{17} \mathrm{NO}_{2}+\mathrm{Na}+278.1157$, found 278.1150.<smiles>Cc1ccc(CCNC(=O)c2ccccc2)cc1</smiles>

$\mathrm{N}$-(4-Metylphenetyl)benzamide (3c)

Yield: $66 \%$; yellowish solid; mp $85-86{ }^{\circ} \mathrm{C}$; IR $v_{\max }(\mathrm{KBr}) / \mathrm{cm}^{-1}: 3324,1640,1544,1313,807,692 ;{ }^{1} \mathrm{H}$
$\operatorname{NMR}\left(\mathrm{CDCl}_{3}, 500 \mathrm{MHz}\right) \delta$ 7.75-7.68 (m, 2H), 7.49-7.46 (m, 1H), 7.42-7.38 (m, 2H), 7.15-7.11 (m, 4H), 6.15 (br s, 1H), 3.70 (qua, $J 7.0 \mathrm{~Hz}, 2 \mathrm{H}$ ), 2.89 (t, $J 7.0 \mathrm{~Hz}, 2 \mathrm{H}$ ), 2.33 (s, 3H); ${ }^{13} \mathrm{C} \mathrm{NMR}\left(\mathrm{CDCl}_{3}, 125 \mathrm{MHz}\right) \delta 167.4,136.0,135.7$, 134.6, 131.3, 129.3, 128.6, 128.4, 126.8, 41.2, 35.2, 21.0; HRMS-ESI $\mathrm{m} / z$ calculated for $\mathrm{C}_{16} \mathrm{H}_{17} \mathrm{NO}+\mathrm{Na}+262.1208$, found 262.1197 .<smiles>Cc1ccccc1CCNC(=O)c1ccccc1</smiles>

\section{$N$-(2-Metylphenetyl)benzamide (3d)}

Yield: 42\%; yellowish solid; mp 76-77 ${ }^{\circ} \mathrm{C}$; IR $v_{\max }(\mathrm{KBr}) / \mathrm{cm}^{-1}: 3306,1632,1536,1309,751,694 ;{ }^{1} \mathrm{H}$ NMR $\left(\mathrm{CDCl}_{3}, 500 \mathrm{MHz}\right) \delta$ 7.72-7.70 (m, 2H), 7.49-7.46 (m, $1 \mathrm{H}), 7.43-7.39(\mathrm{~m}, 2 \mathrm{H}), 7.19-7.14(\mathrm{~m}, 4 \mathrm{H}), 6.25(\mathrm{br} \mathrm{s}, 1 \mathrm{H})$, 3.68 (qua, $J 7.0 \mathrm{~Hz}, 2 \mathrm{H}$ ), 2.95 (t, $J 7.0 \mathrm{~Hz}, 2 \mathrm{H}$ ), 2.37 (s, $3 \mathrm{H}) ;{ }^{13} \mathrm{C} \mathrm{NMR}\left(\mathrm{CDCl}_{3}, 125 \mathrm{MHz}\right) \delta 167.6,137.0,136.4$, 134.6, 131.4, 130.5, 129.4, 128.5, 126.9, 126.7, 126.1, 40.0, 33.1, 19.3; HRMS-ESI $m / z$ calculated for $\mathrm{C}_{16} \mathrm{H}_{17} \mathrm{NO}+\mathrm{Na}+$ 262.1208 , found 262.1214 .<smiles>O=C(NCCc1cccc(C(F)(F)F)c1)c1ccccc1</smiles>

$\mathrm{N}$-(3-(Trifluoromethyl)phenethyl)benzamide (3e)

Yield: 35\%; yellowish solid, $\mathrm{mp} 81-82{ }^{\circ} \mathrm{C}$; IR $v_{\max }(\mathrm{KBr}) / \mathrm{cm}^{-1}: 3304,1629,1555,1337,1170,801,699$; ${ }^{1} \mathrm{H}$ NMR $\left(\mathrm{CDCl}_{3}, 500 \mathrm{MHz}\right) \delta$ 7.71-7.69 (m, 2H), 7.50-7.46 (m, 3H), 7.44-7.38 (m, 4H), 6.34 (br s, 1H), 3.70 (qua, $J$ $7.0 \mathrm{~Hz}, 2 \mathrm{H}), 2.99$ (t, $J 7.0 \mathrm{~Hz}, 2 \mathrm{H}) ;{ }^{13} \mathrm{C}$ NMR $\left(\mathrm{CDCl}_{3}, 125\right.$ MHz) $\delta 167.7,139.9,134.5,132.3,131.6,131.0$ (qua, $J$ $32.0 \mathrm{~Hz}), 129.2,128.6,126.8,125.6,125.5,123.5,123.4$, 41.0, 35.6; HRMS-ESI $\mathrm{m} / \mathrm{z}$ calculated for $\mathrm{C}_{16} \mathrm{H}_{14} \mathrm{~F}_{3} \mathrm{NO}+$ $\mathrm{Na}^{+} 316.0925$, found 316.0927.<smiles>O=C(NCCCc1ccccc1)c1ccccc1</smiles>

$N$-(3-Phenylproyl)benzamide (3f)

Yield: 35\%; yellowish oil, IR $v_{\max }($ film $) / \mathrm{cm}^{-1}: 3318$, $1638,1578,1309,1181,698 ;{ }^{1} \mathrm{H}$ NMR $\left(\mathrm{CDCl}_{3}, 500 \mathrm{MHz}\right)$ $\delta$ 7.69-7.67 (m, 2H), 7.45-7.41 (m, 1H), 7.36-7.33 (m, $2 \mathrm{H}), 7.27-7.23(\mathrm{~m}, 2 \mathrm{H}), 7.18-7.16(\mathrm{~m}, 3 \mathrm{H}), 6.43$ (br s, 1H), 3.44 (qua, $J 6.0 \mathrm{~Hz}, 2 \mathrm{H}$ ), 2.67 (t, $J .5 \mathrm{~Hz}, 2 \mathrm{H}$ ), 1.92 (qui, $J 7.5 \mathrm{~Hz}, 2 \mathrm{H}) ;{ }^{13} \mathrm{C} \mathrm{NMR}\left(\mathrm{CDCl}_{3}, 125 \mathrm{MHz}\right) \delta 167.5$, 141.4, 134.5, 131.1, 128.4, 128.3, 128.2, 126.8, 125.9, 39.7, 33.4, 31.0; HRMS-ESI $m / z$ calculated for $\mathrm{C}_{16} \mathrm{H}_{17} \mathrm{NO}+\mathrm{Na}^{+}$ 262.1208, found 262.1204 . 


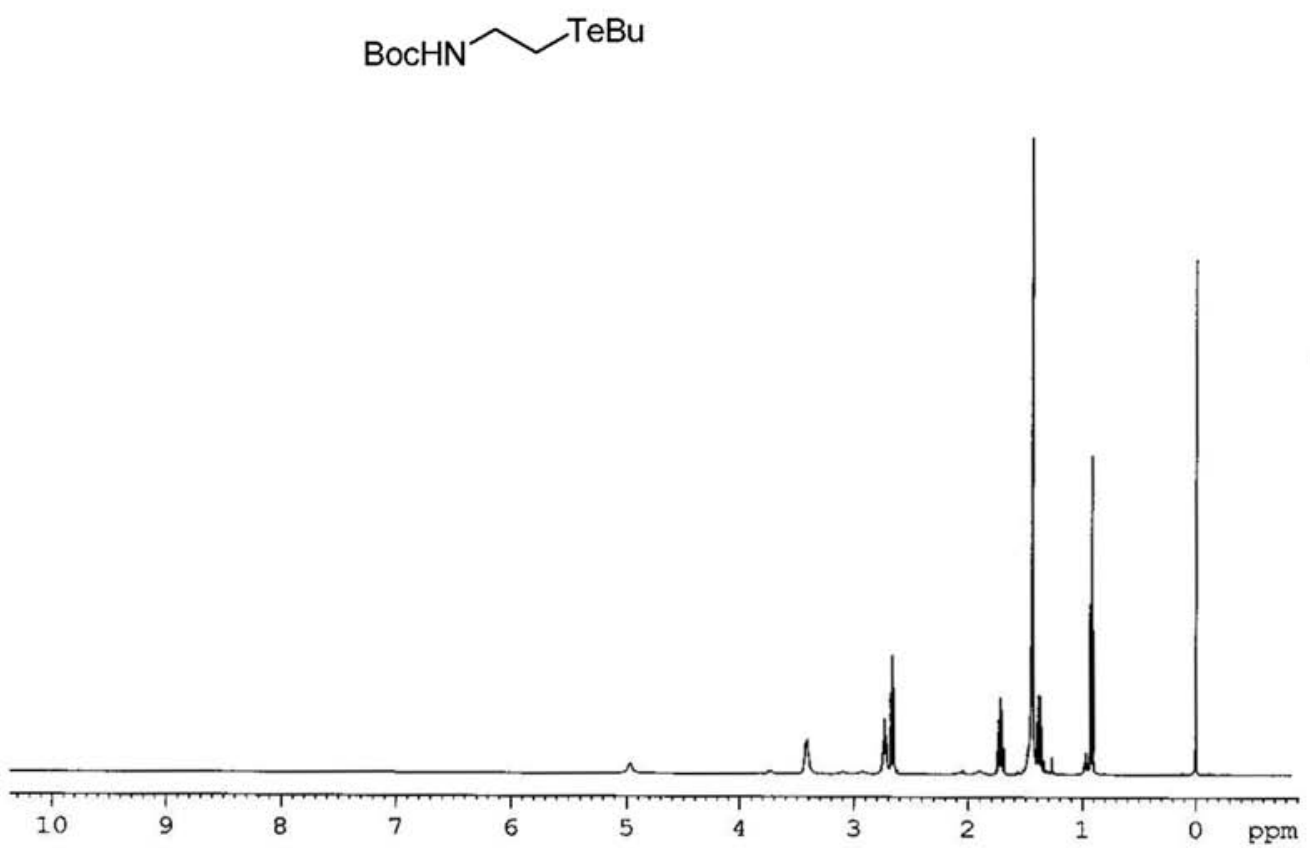

Figure S1. 'H NMR $\left(500 \mathrm{MHz}, \mathrm{CDCl}_{3}\right)$ spectrum of $\mathbf{1 a}$.
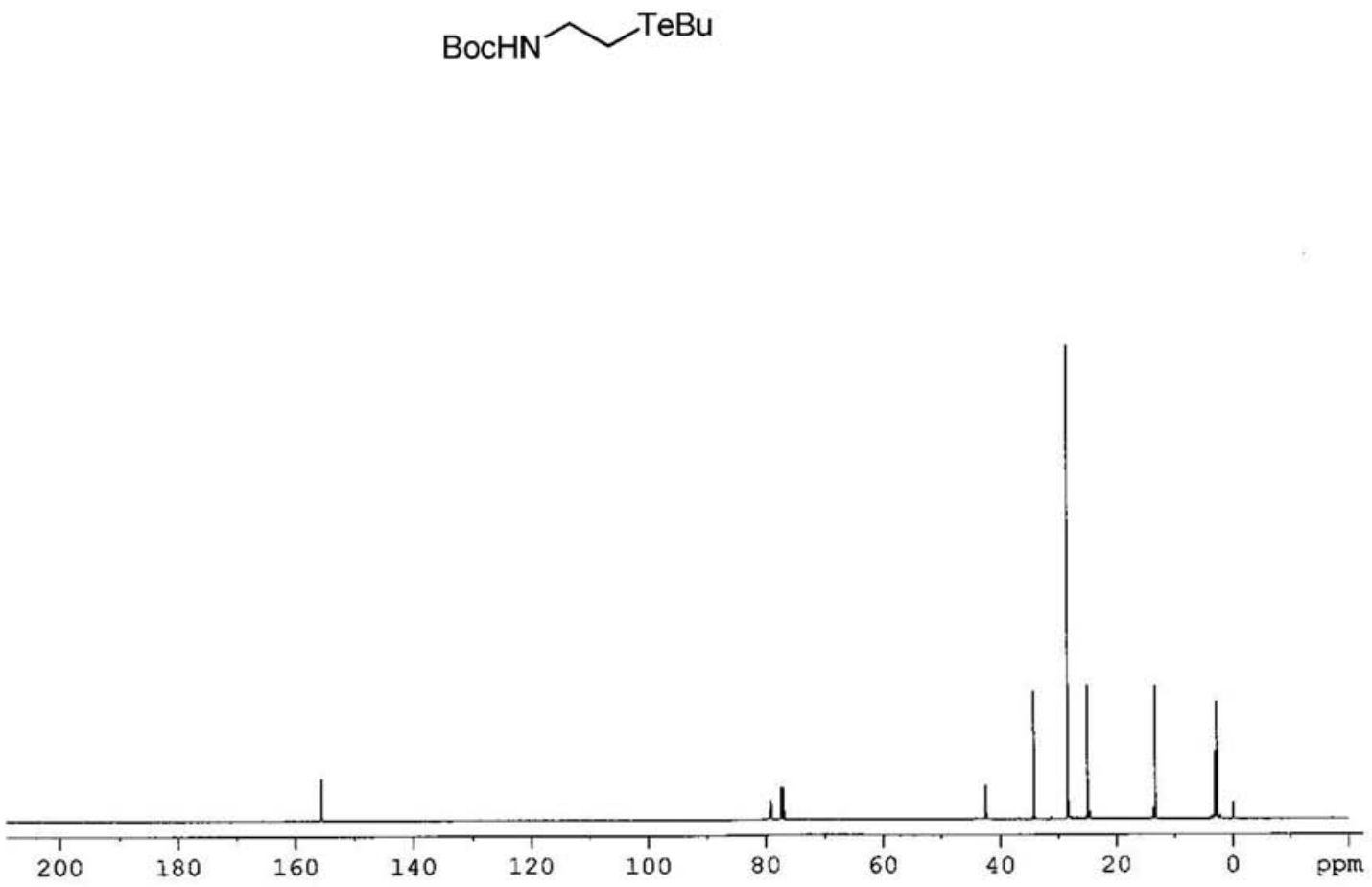

Figure S2. ${ }^{13} \mathrm{C}$ NMR $\left(125 \mathrm{MHz}, \mathrm{CDCl}_{3}\right)$ spectrum of $\mathbf{1 a}$. 


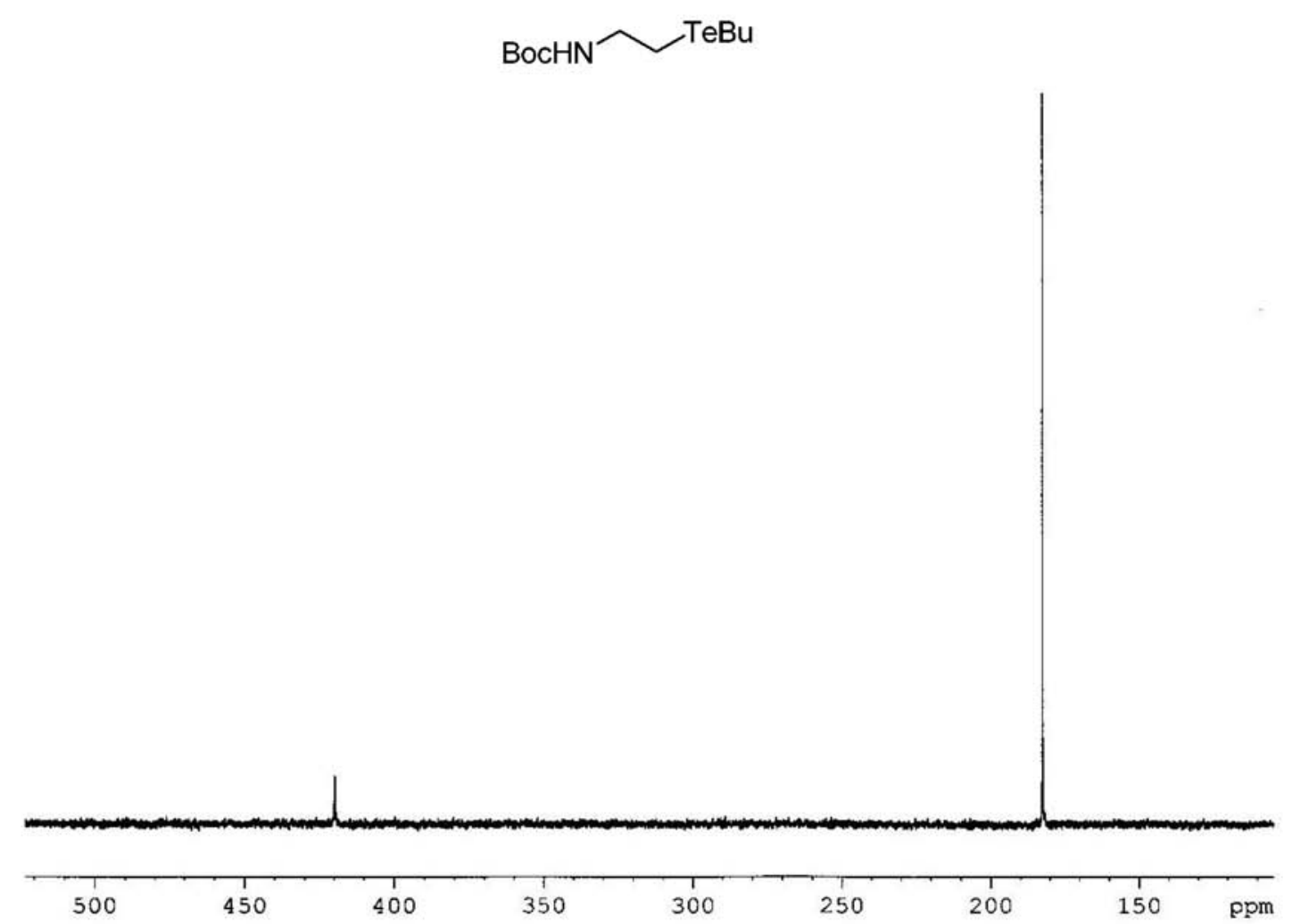

Figure S3. ${ }^{125} \mathrm{Te} \mathrm{NMR}\left(157 \mathrm{MHz}, \mathrm{CDCl}_{3}\right)$ spectrum of $\mathbf{1 a}$.

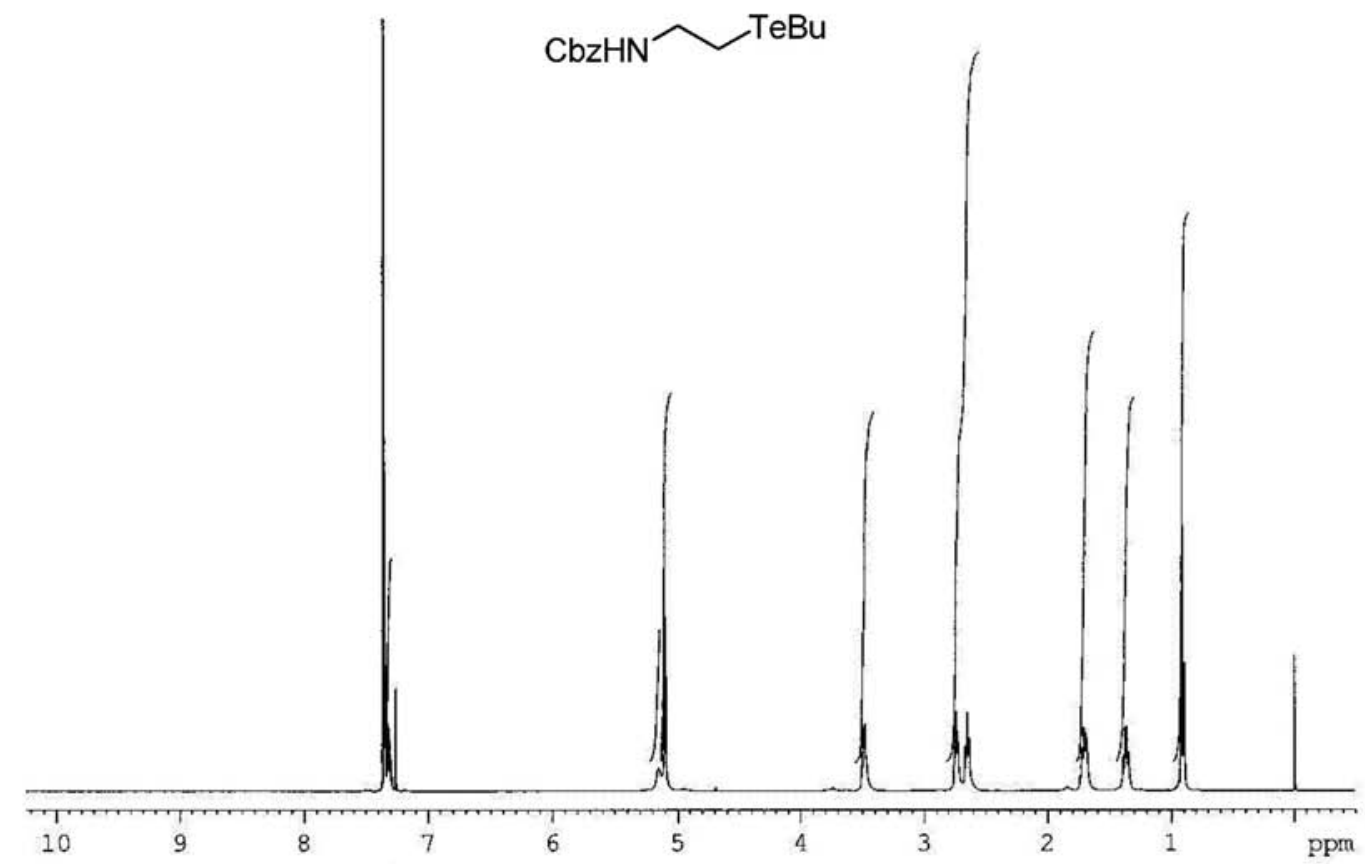

Figure S4. ${ }^{1} \mathrm{H}$ NMR $\left(500 \mathrm{MHz}, \mathrm{CDCl}_{3}\right)$ spectrum of $\mathbf{1 b}$. 


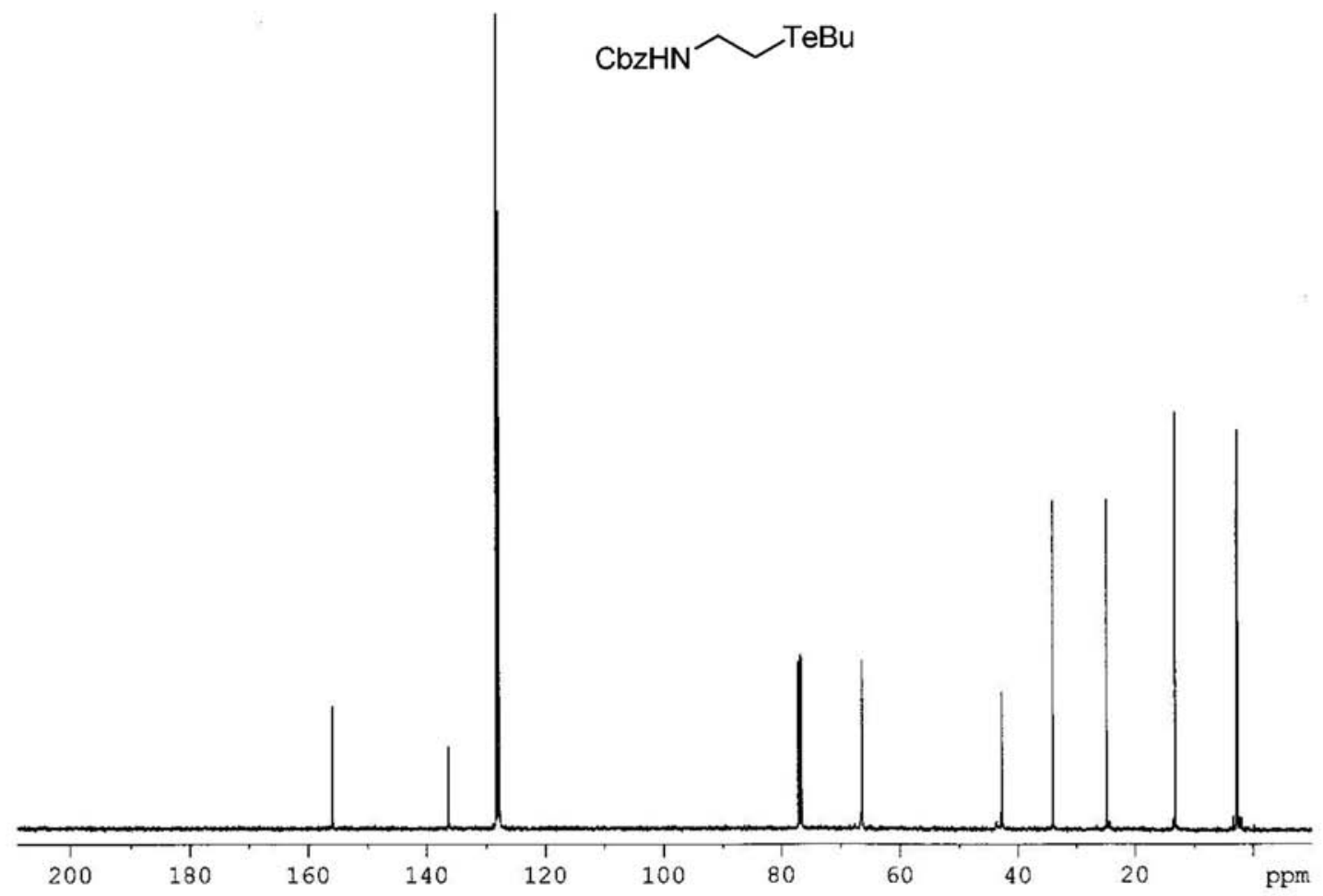

Figure S5. ${ }^{13} \mathrm{C}$ NMR $\left(125 \mathrm{MHz}, \mathrm{CDCl}_{3}\right)$ spectrum of $\mathbf{1 b}$.

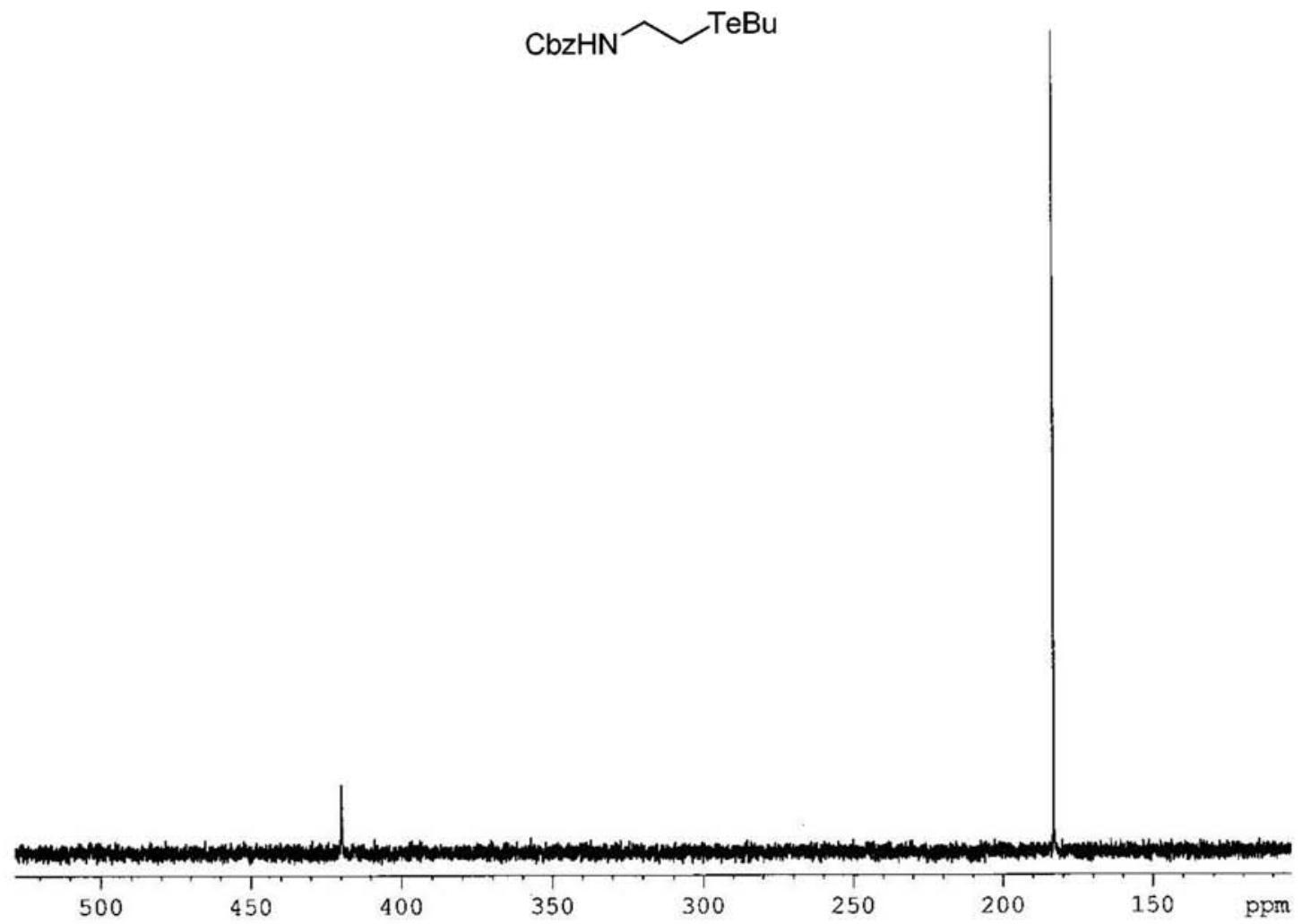

Figure S6. ${ }^{125}$ Te NMR $\left(157 \mathrm{MHz}, \mathrm{CDCl}_{3}\right)$ spectrum of $\mathbf{1 b}$. 


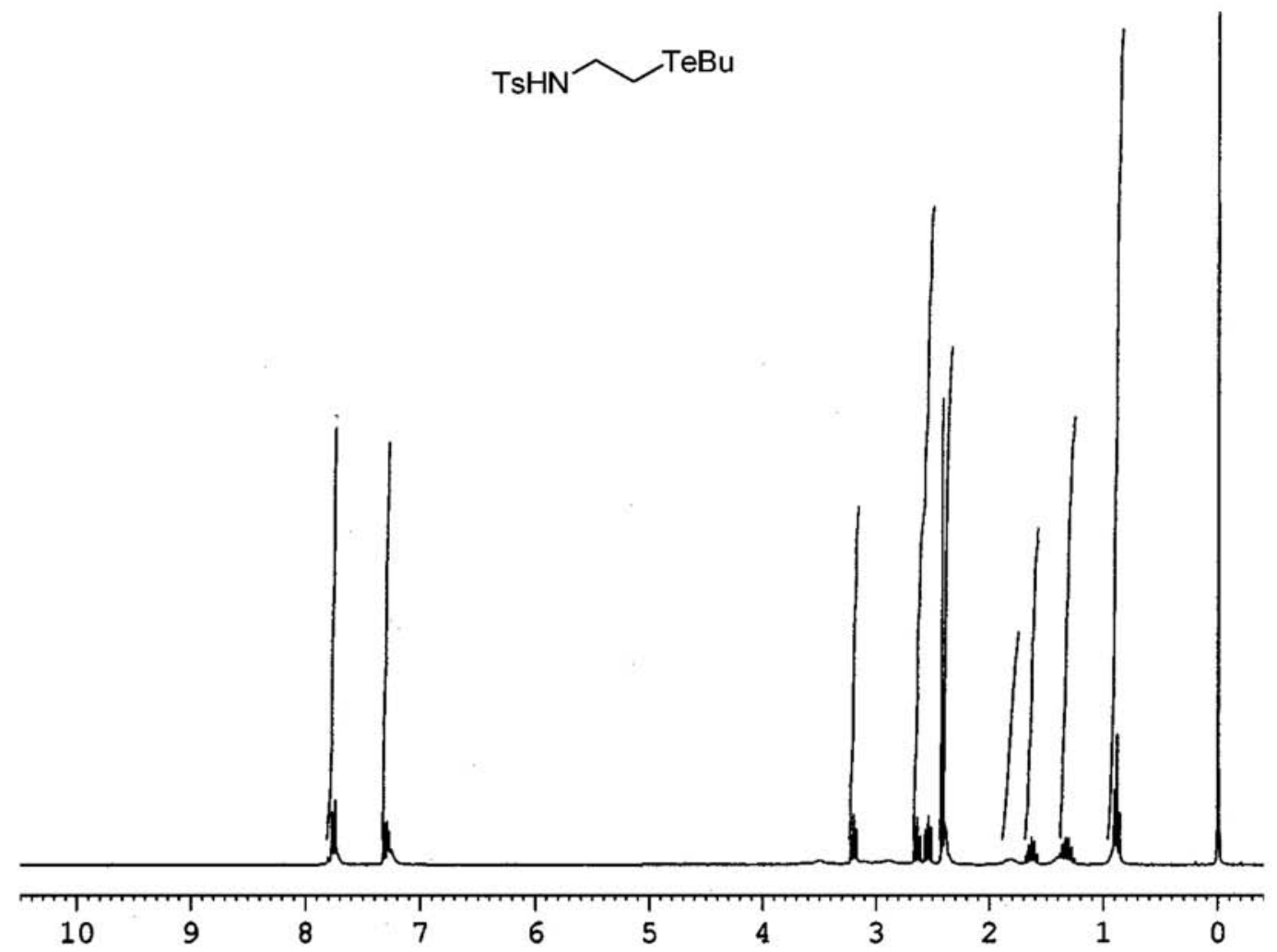

Figure S7. ${ }^{1} \mathrm{H}$ NMR $\left(300 \mathrm{MHz}, \mathrm{CDCl}_{3}\right)$ spectrum of $\mathbf{1 c}$.
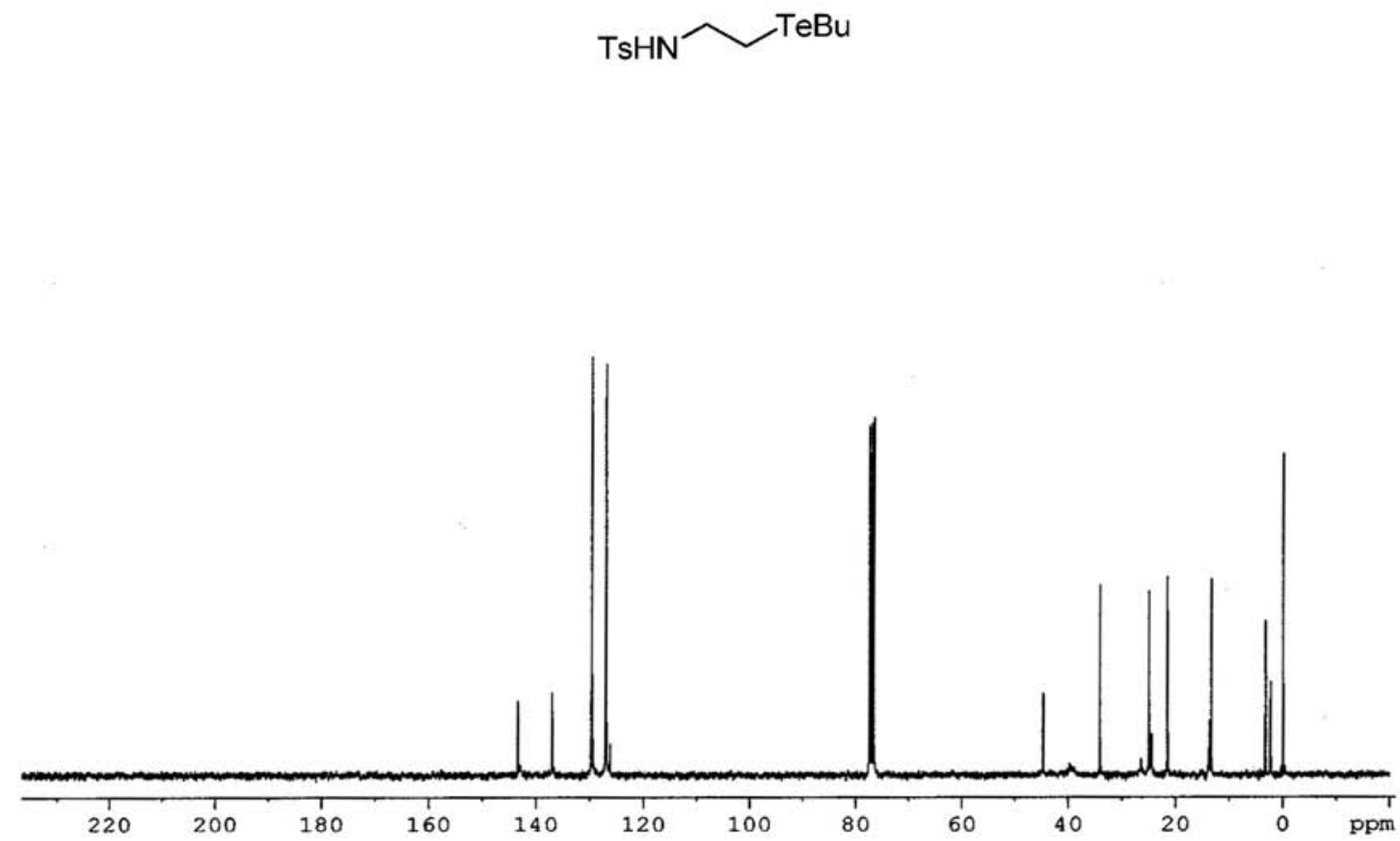

Figure S8. ${ }^{13} \mathrm{C}$ NMR $\left(75 \mathrm{MHz}, \mathrm{CDCl}_{3}\right)$ spectrum of $\mathbf{1 c}$. 


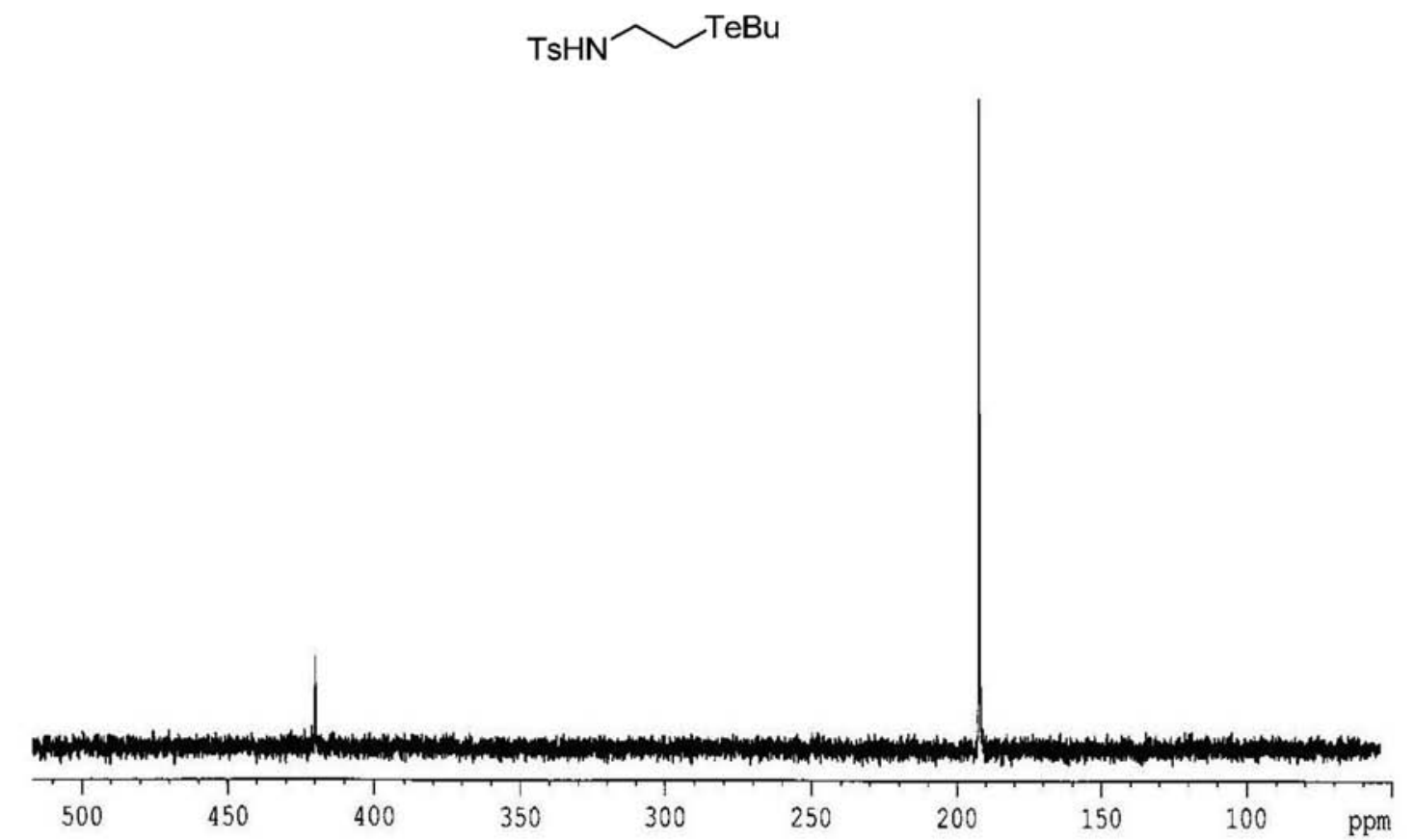

Figure S9. ${ }^{125} \mathrm{Te} \mathrm{NMR}\left(157 \mathrm{MHz}, \mathrm{CDCl}_{3}\right)$ spectrum of $\mathbf{1 c}$.

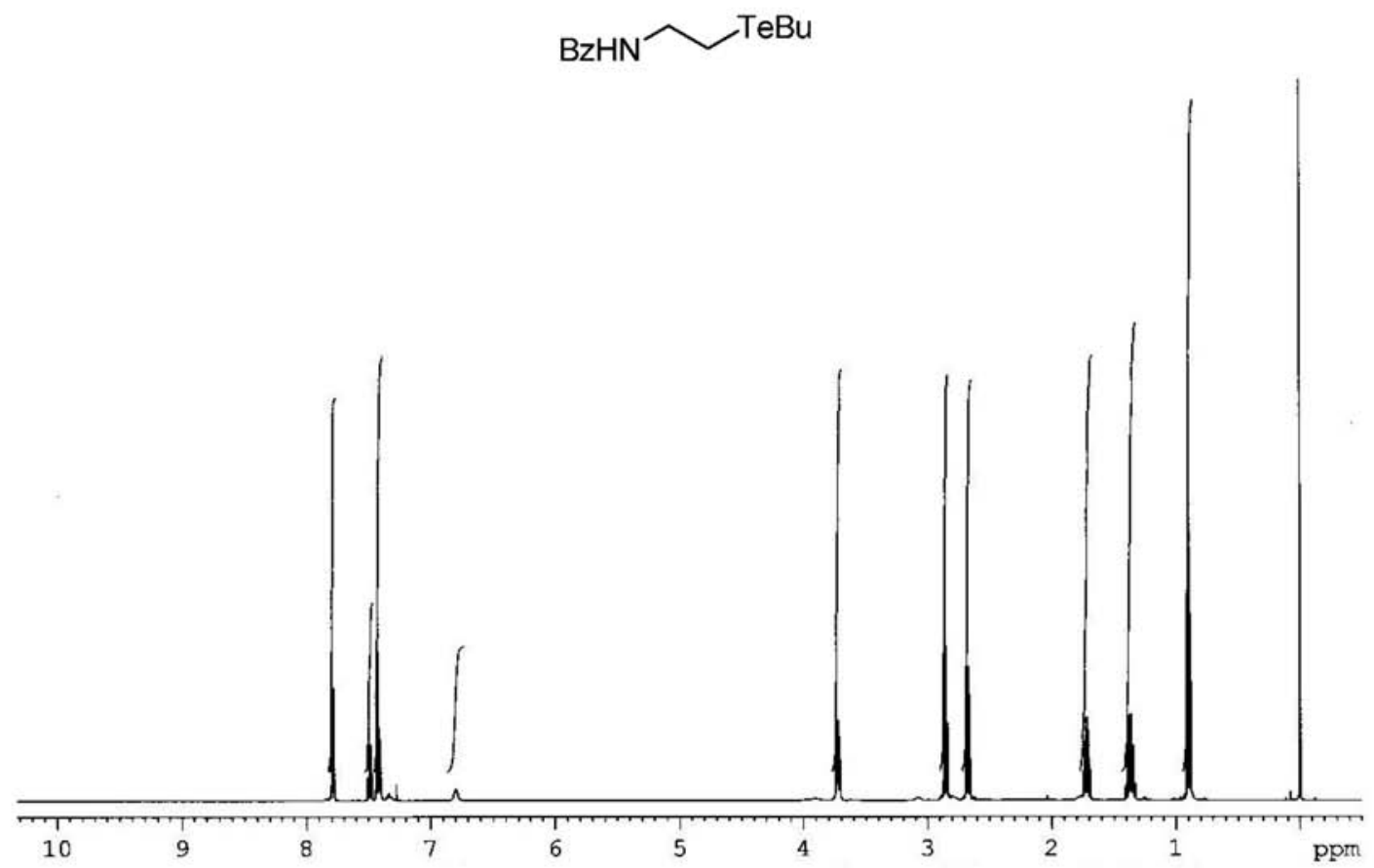

Figure S10. ${ }^{1} \mathrm{H}$ NMR $\left(500 \mathrm{MHz}, \mathrm{CDCl}_{3}\right)$ spectrum of $\mathbf{1 d}$. 


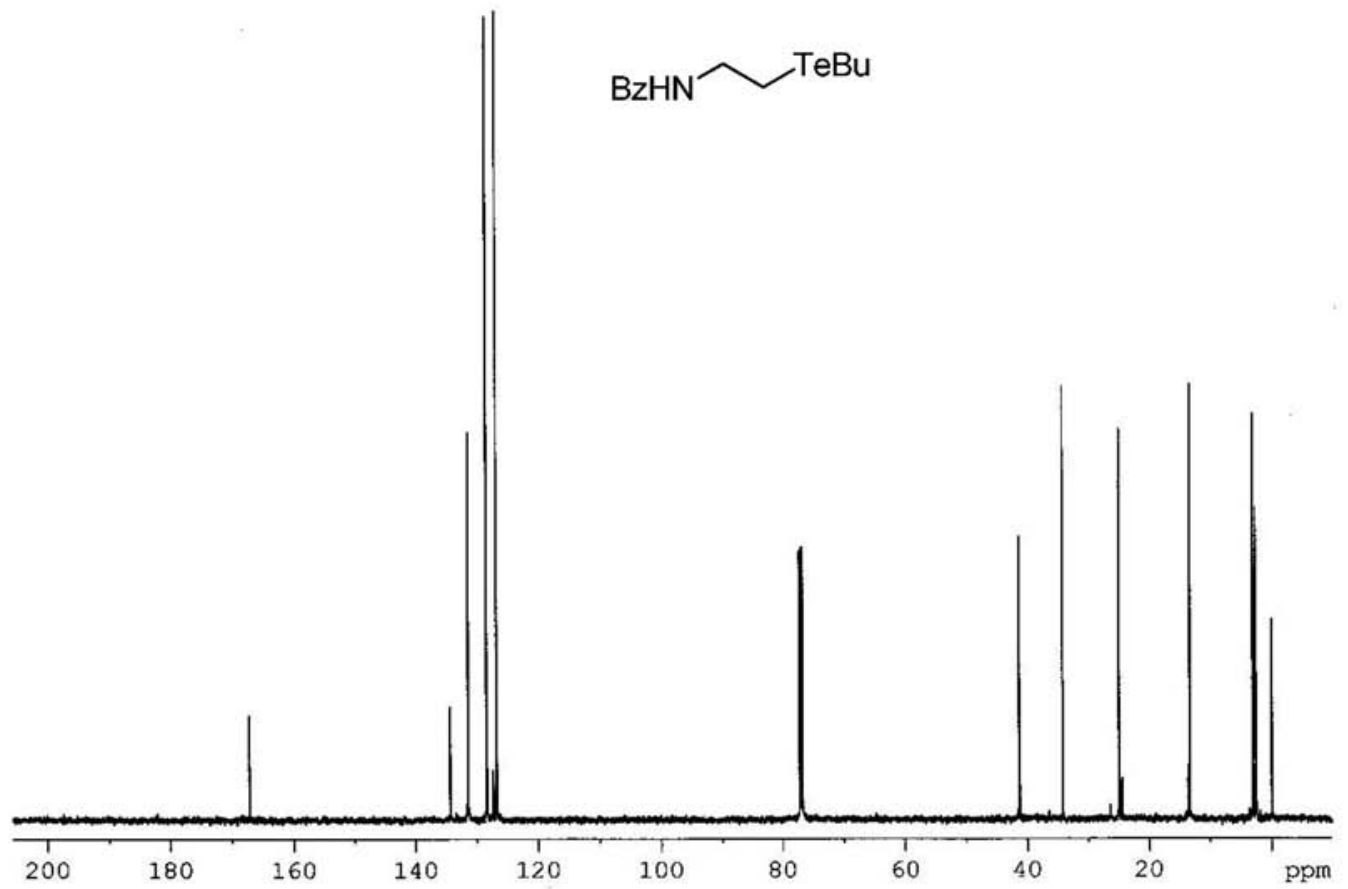

Figure S11. ${ }^{13} \mathrm{C}$ NMR (125 MHz, $\mathrm{CDCl}_{3}$ ) spectrum of 1d.

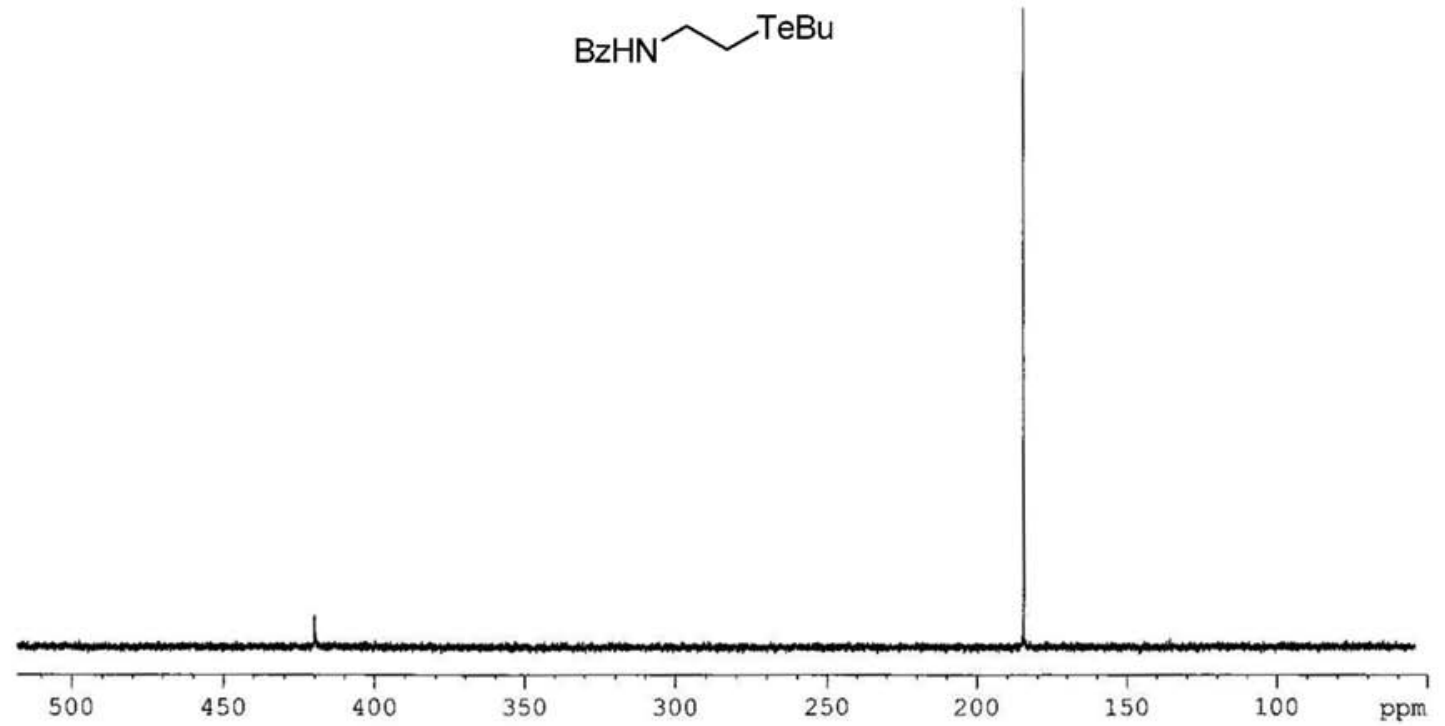

Figure S12. ${ }^{125} \mathrm{Te} \mathrm{NMR}\left(157 \mathrm{MHz}, \mathrm{CDCl}_{3}\right)$ spectrum of $\mathbf{1 d}$. 


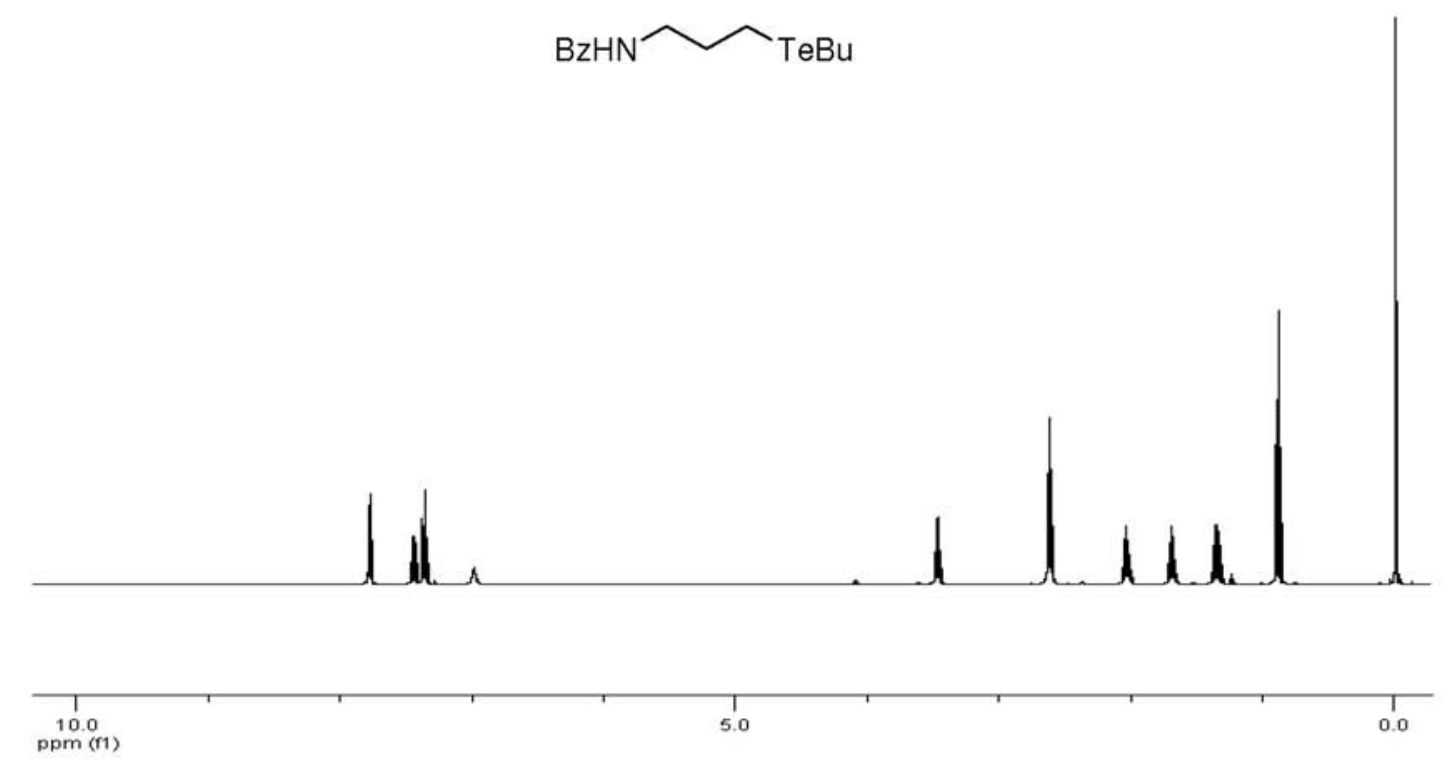

Figure S13. ${ }^{1} \mathrm{H} \mathrm{NMR}\left(500 \mathrm{MHz}, \mathrm{CDCl}_{3}\right)$ spectrum of $\mathbf{1 e}$.
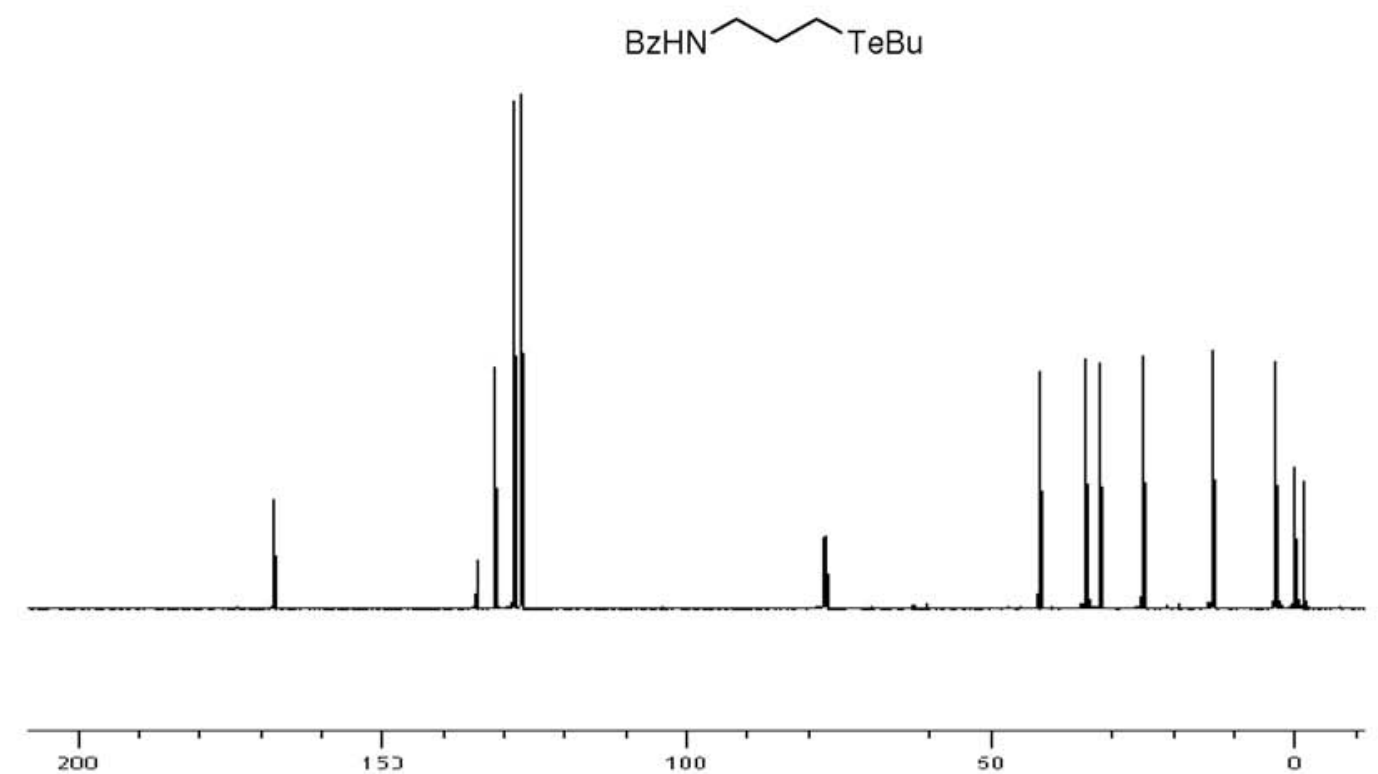

Figure S14. ${ }^{13} \mathrm{C}$ NMR $\left(125 \mathrm{MHz}, \mathrm{CDCl}_{3}\right)$ spectrum of $\mathbf{1 e .}$ 


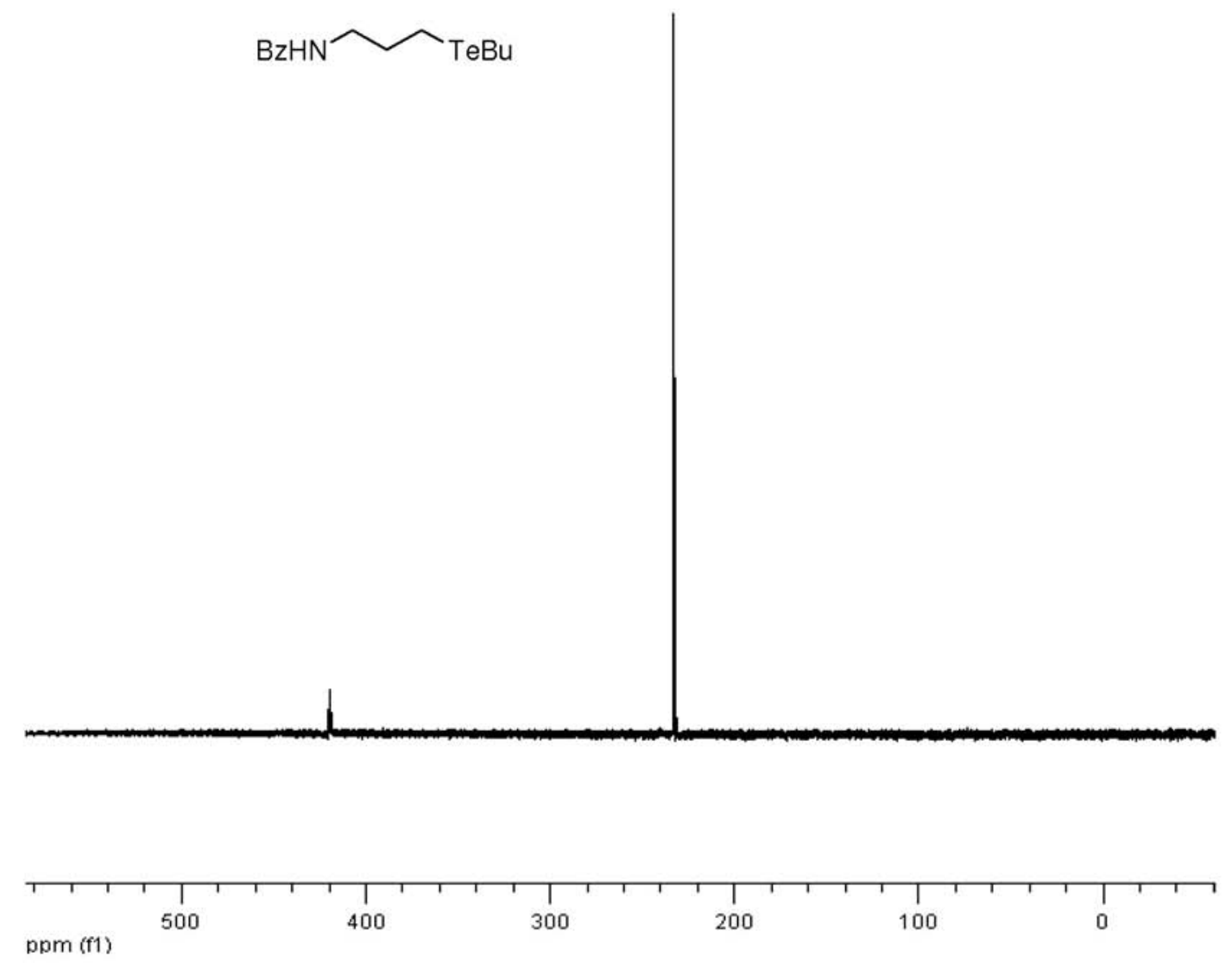

Figure S15. ${ }^{125} \mathrm{Te} \mathrm{NMR}\left(157 \mathrm{MHz}, \mathrm{CDCl}_{3}\right)$ spectrum of $\mathbf{1 e}$.
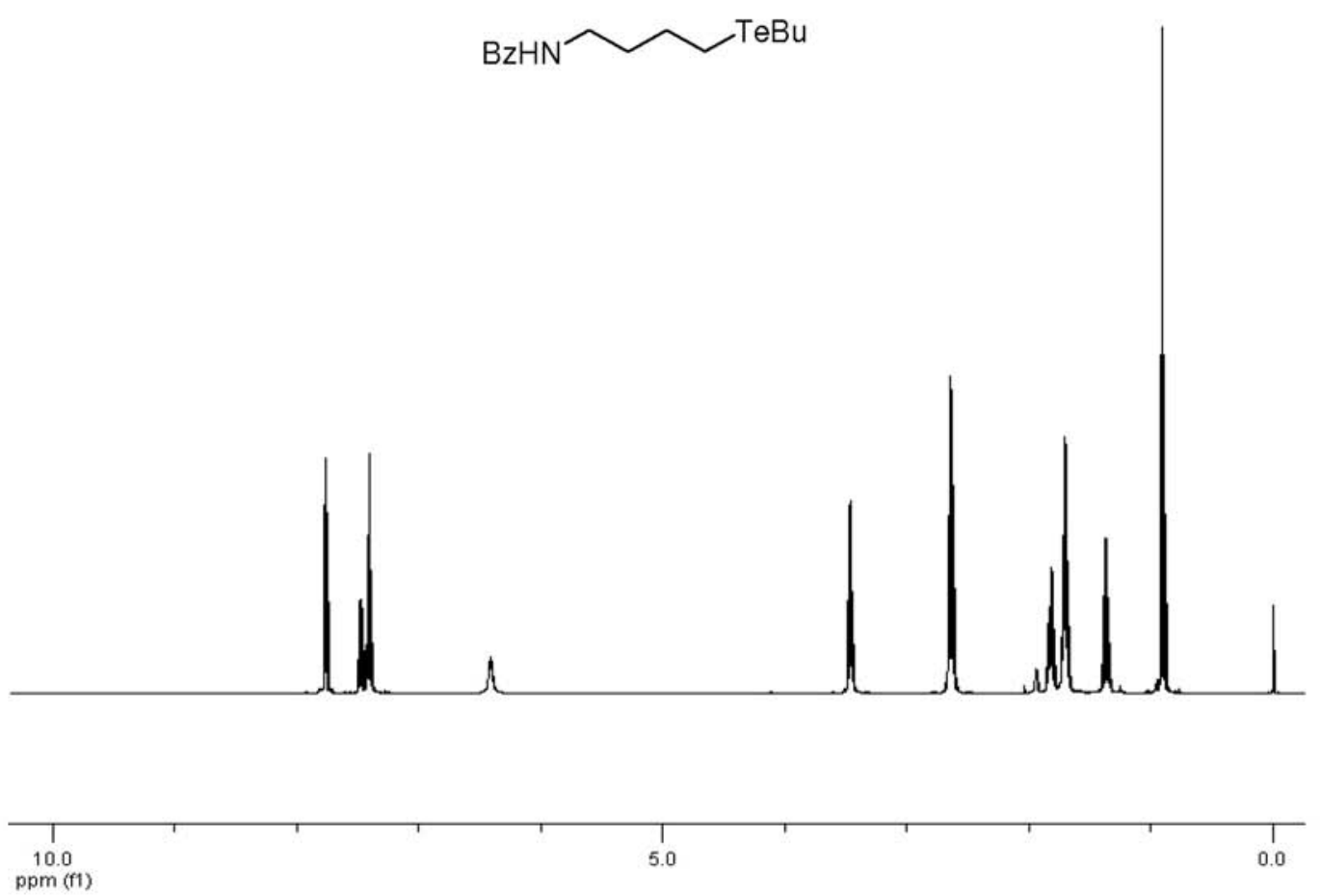

Figure S16. ${ }^{1} \mathrm{H}$ NMR $\left(500 \mathrm{MHz}, \mathrm{CDCl}_{3}\right)$ spectrum of $\mathbf{1 f}$. 

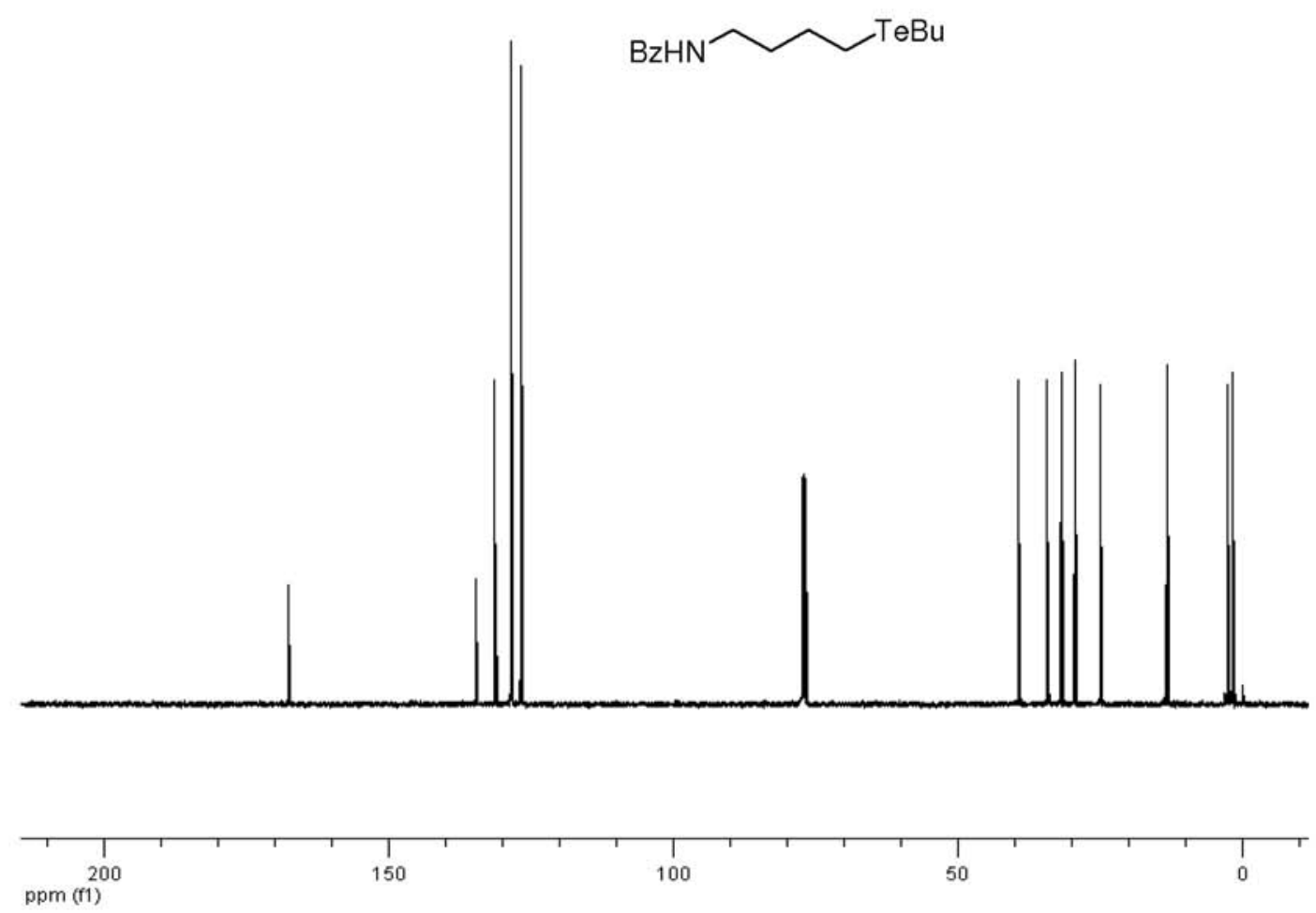

Figure S17. ${ }^{13} \mathrm{C}$ NMR $\left(125 \mathrm{MHz}, \mathrm{CDCl}_{3}\right)$ spectrum of $\mathbf{1 f}$.
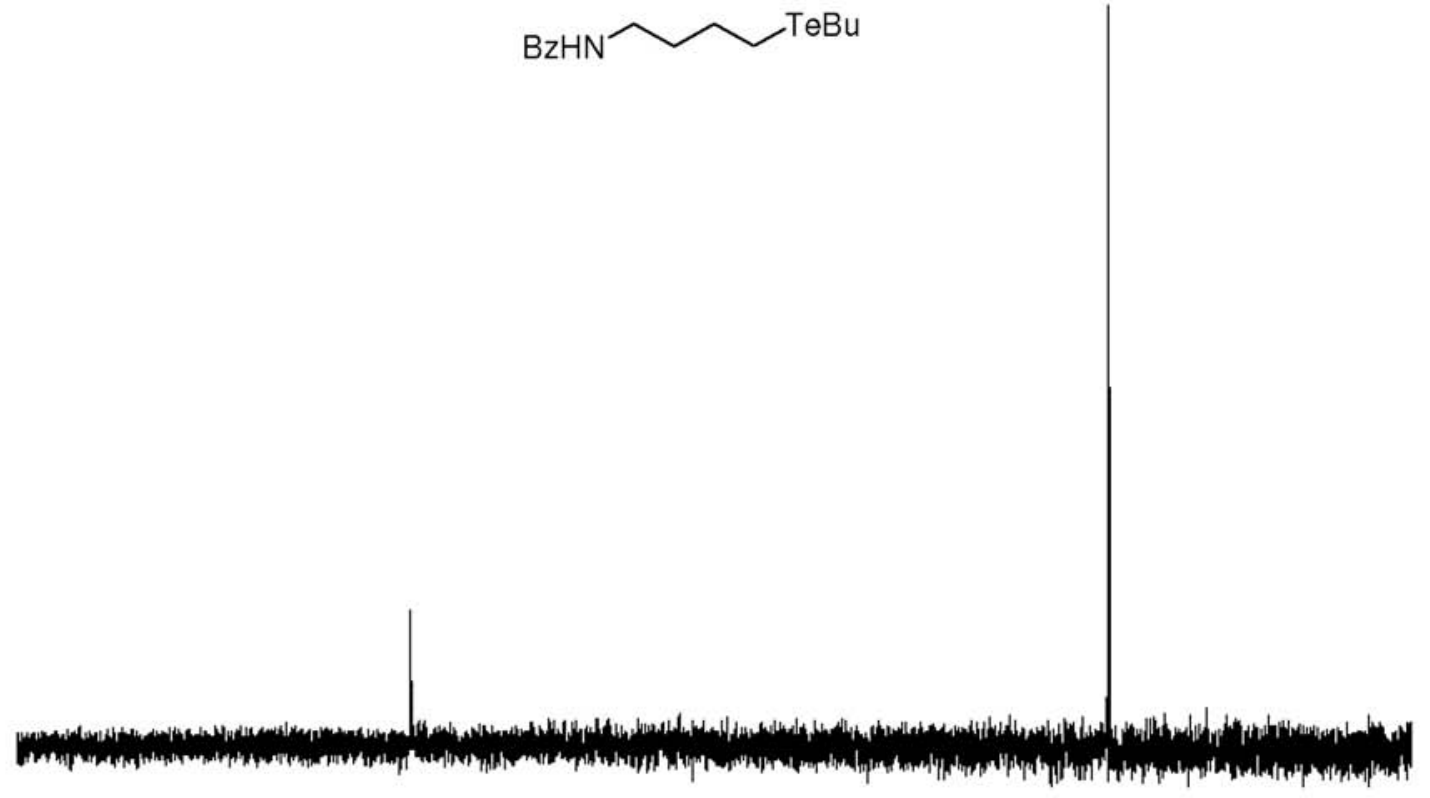

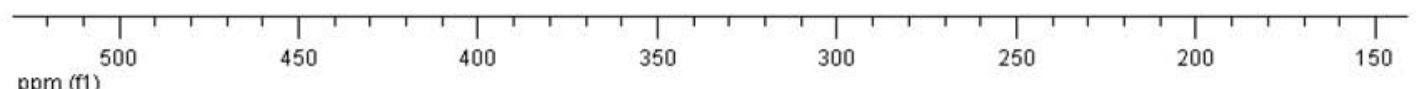

Figure S18. ${ }^{125} \mathrm{Te} \mathrm{NMR}\left(157 \mathrm{MHz}, \mathrm{CDCl}_{3}\right)$ spectrum of $\mathbf{1 f}$. 


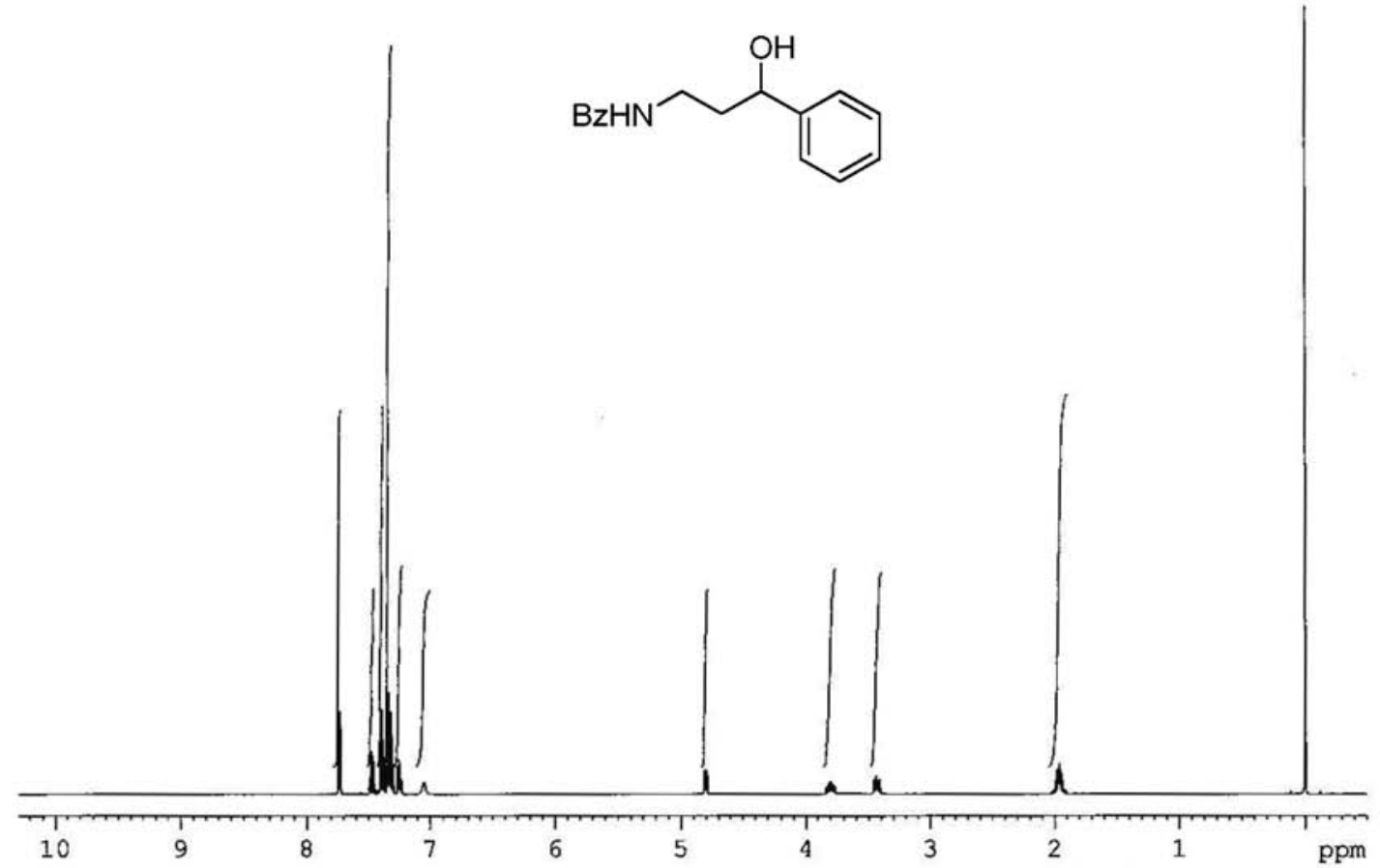

Figure S19. ${ }^{1} \mathrm{H} \mathrm{NMR}\left(500 \mathrm{MHz}, \mathrm{CDCl}_{3}\right)$ spectrum of $2 \mathbf{a}$.<smiles>O=C(NCCC(O)c1ccccc1)c1ccccc1</smiles>

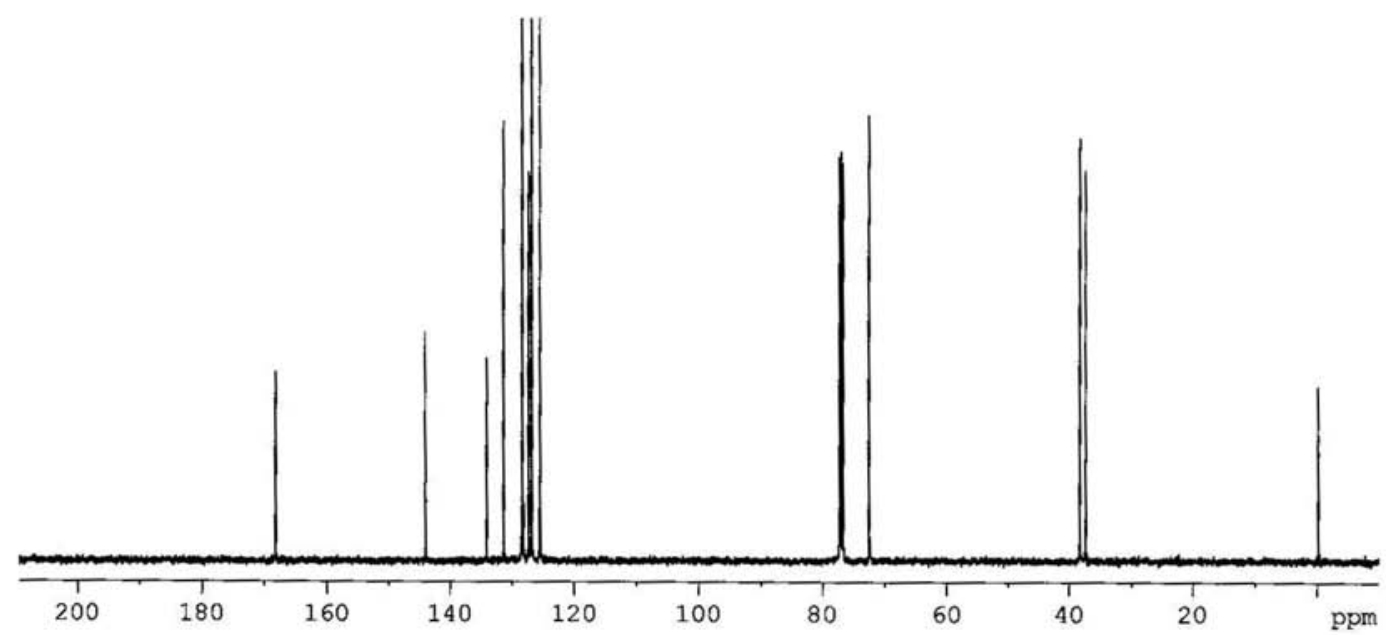

Figure S20. ${ }^{13} \mathrm{C}$ NMR $\left(125 \mathrm{MHz}, \mathrm{CDCl}_{3}\right)$ spectrum of $\mathbf{2 a}$. 


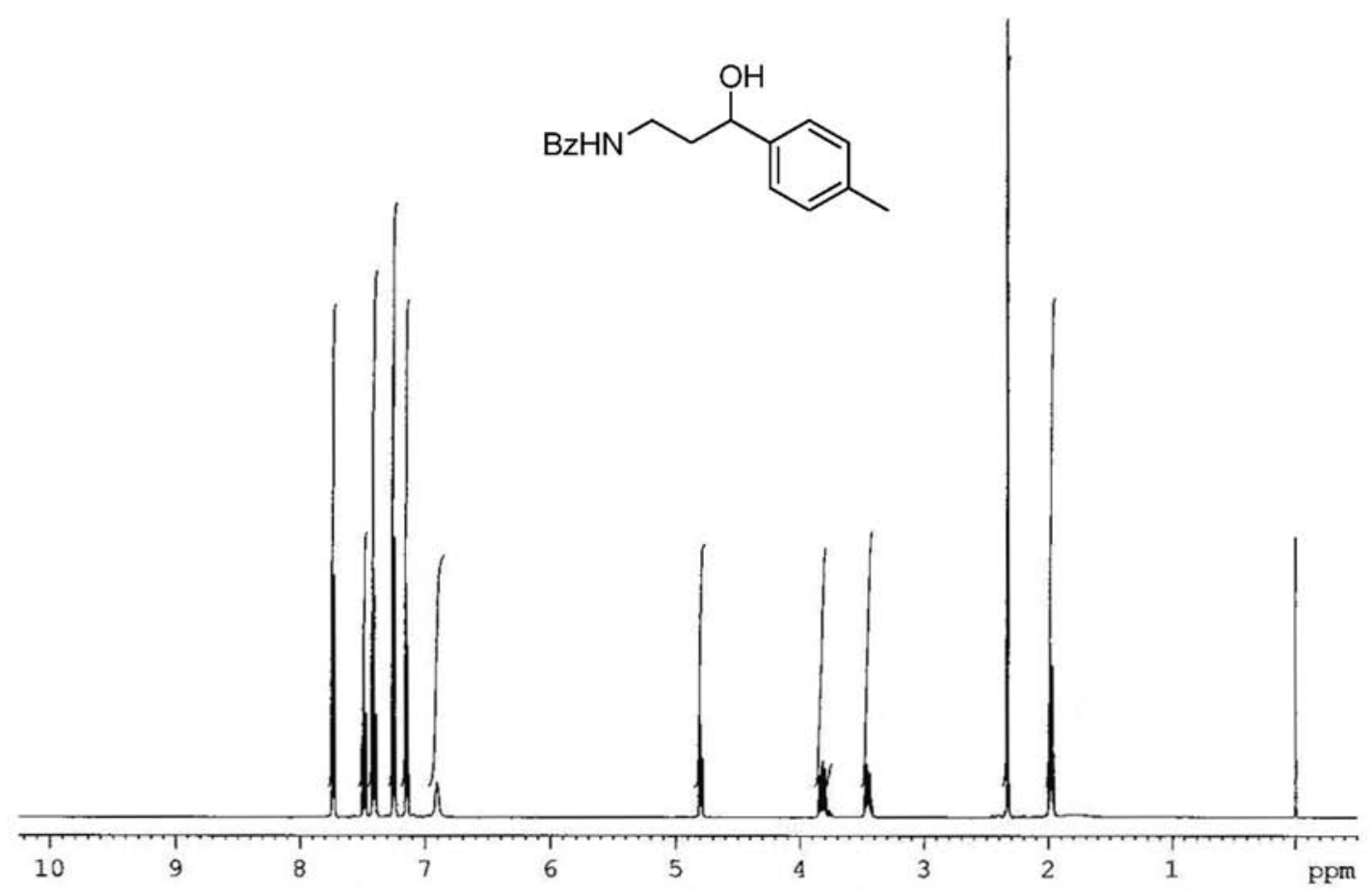

Figure S21. ${ }^{1} \mathrm{H}$ NMR $\left(500 \mathrm{MHz}, \mathrm{CDCl}_{3}\right)$ spectrum of $\mathbf{2 b}$.

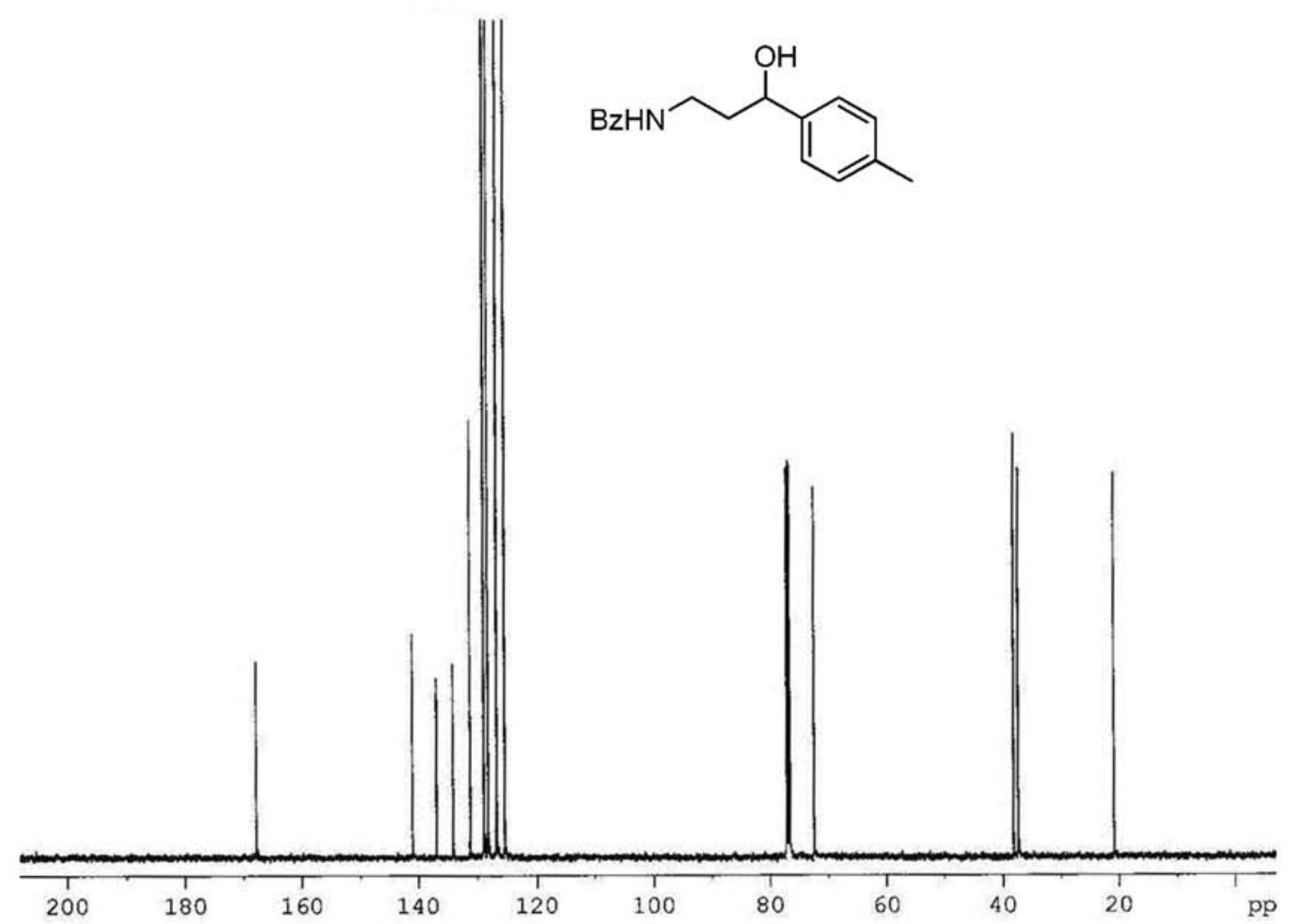

Figure S22. ${ }^{13} \mathrm{C}$ NMR $\left(125 \mathrm{MHz}, \mathrm{CDCl}_{3}\right)$ spectrum of $\mathbf{2 b}$. 


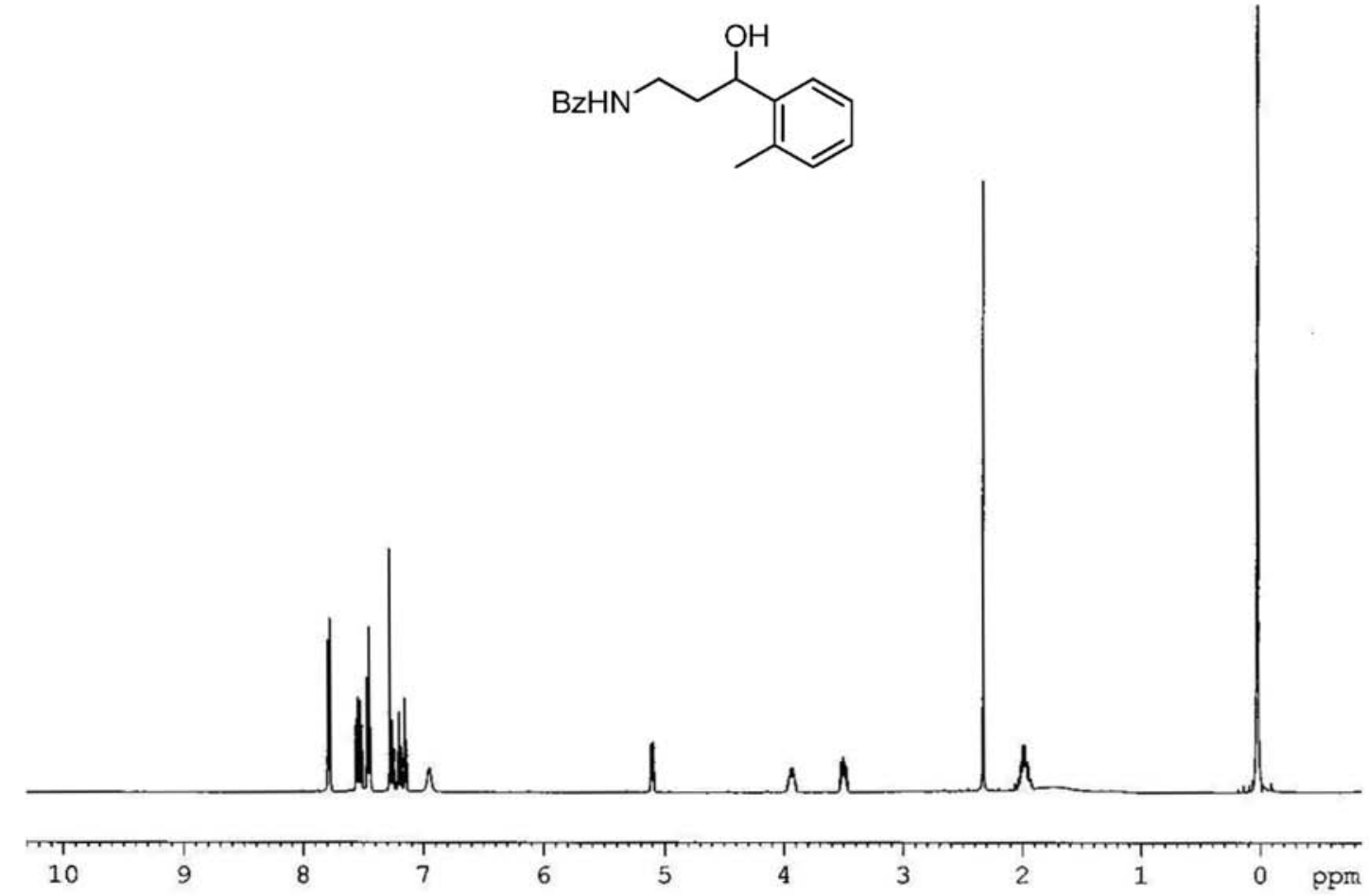

Figure S23. ${ }^{1} \mathrm{H} \mathrm{NMR}\left(500 \mathrm{MHz}, \mathrm{CDCl}_{3}\right)$ spectrum of $2 \mathbf{c}$.

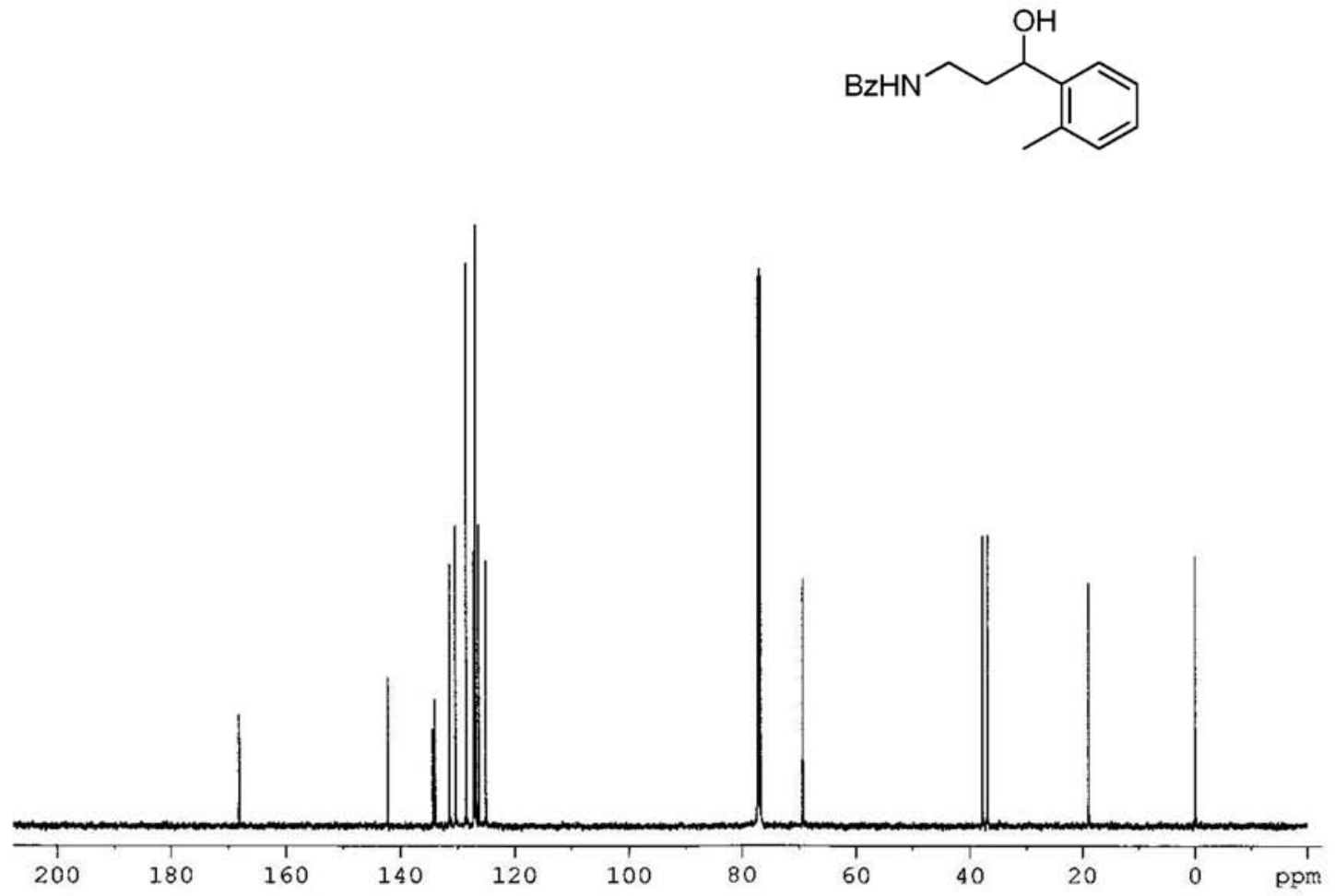

Figure S24. ${ }^{13} \mathrm{C}$ NMR $\left(125 \mathrm{MHz}, \mathrm{CDCl}_{3}\right)$ spectrum of 2 c. 


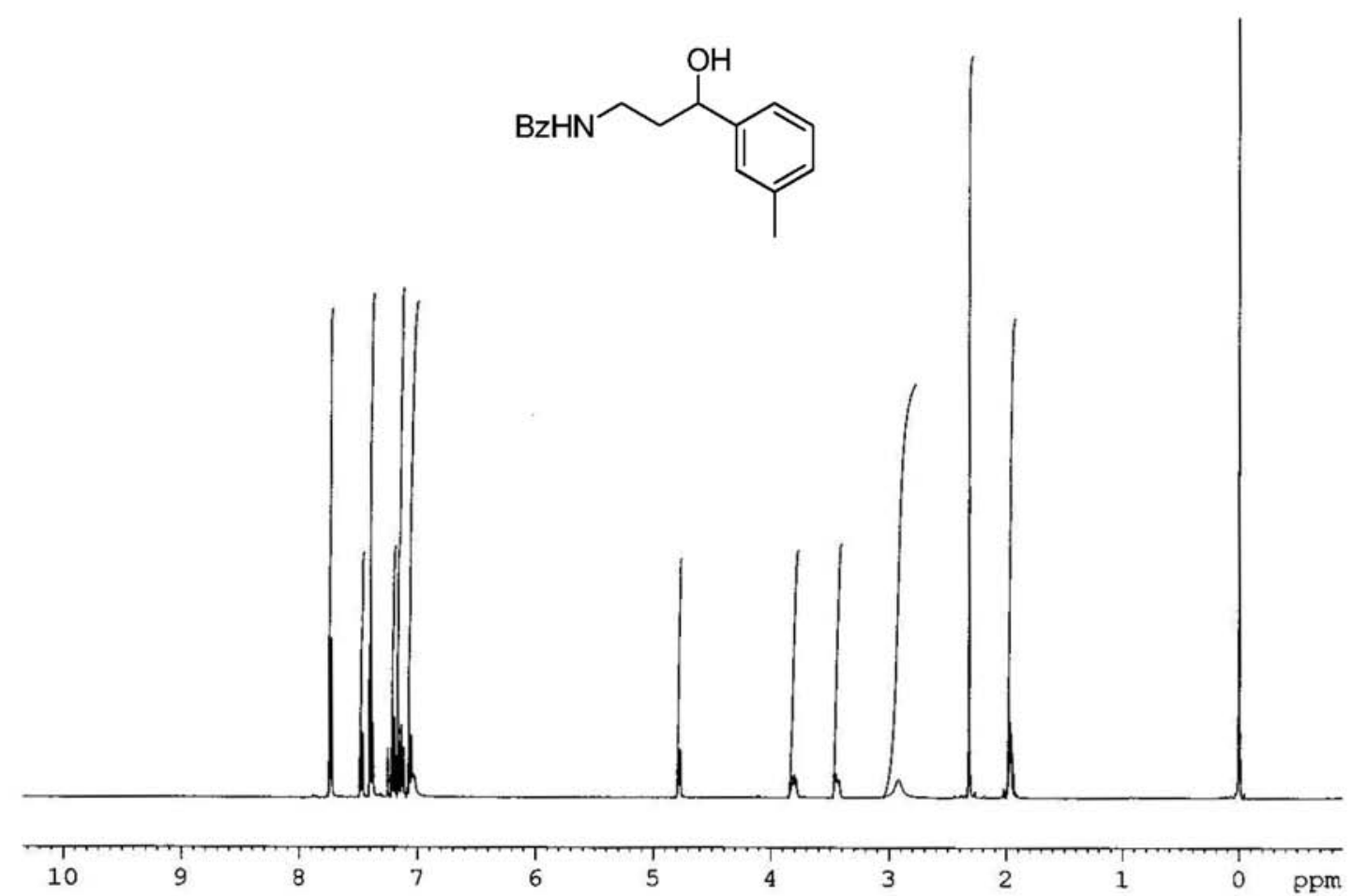

Figure S25. ${ }^{1} \mathrm{H}$ NMR $\left(500 \mathrm{MHz}, \mathrm{CDCl}_{3}\right)$ spectrum of $\mathbf{2 d}$.

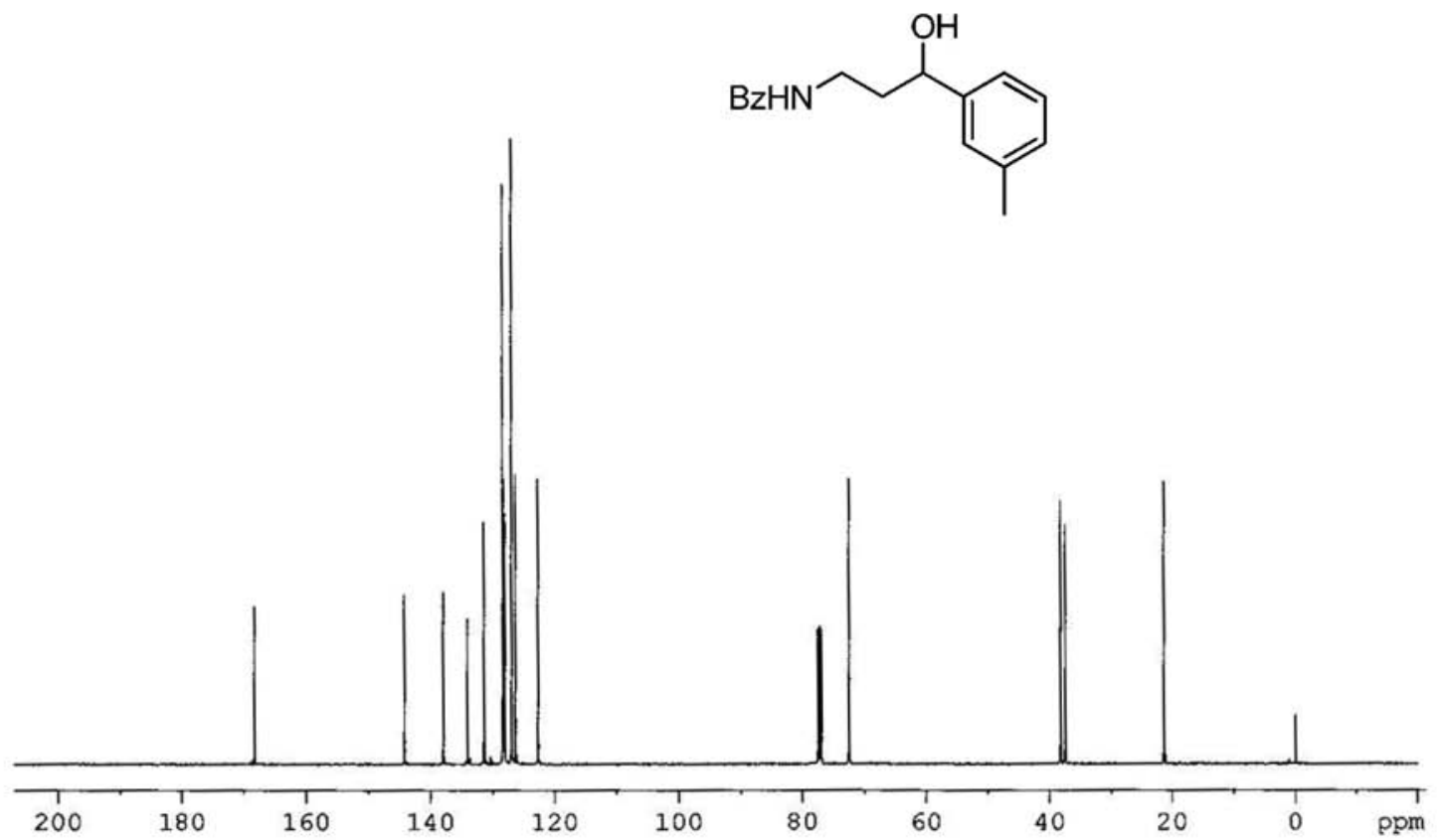

Figure S26. ${ }^{13} \mathrm{C}$ NMR $\left(125 \mathrm{MHz}, \mathrm{CDCl}_{3}\right)$ spectrum of $\mathbf{2 d}$. 


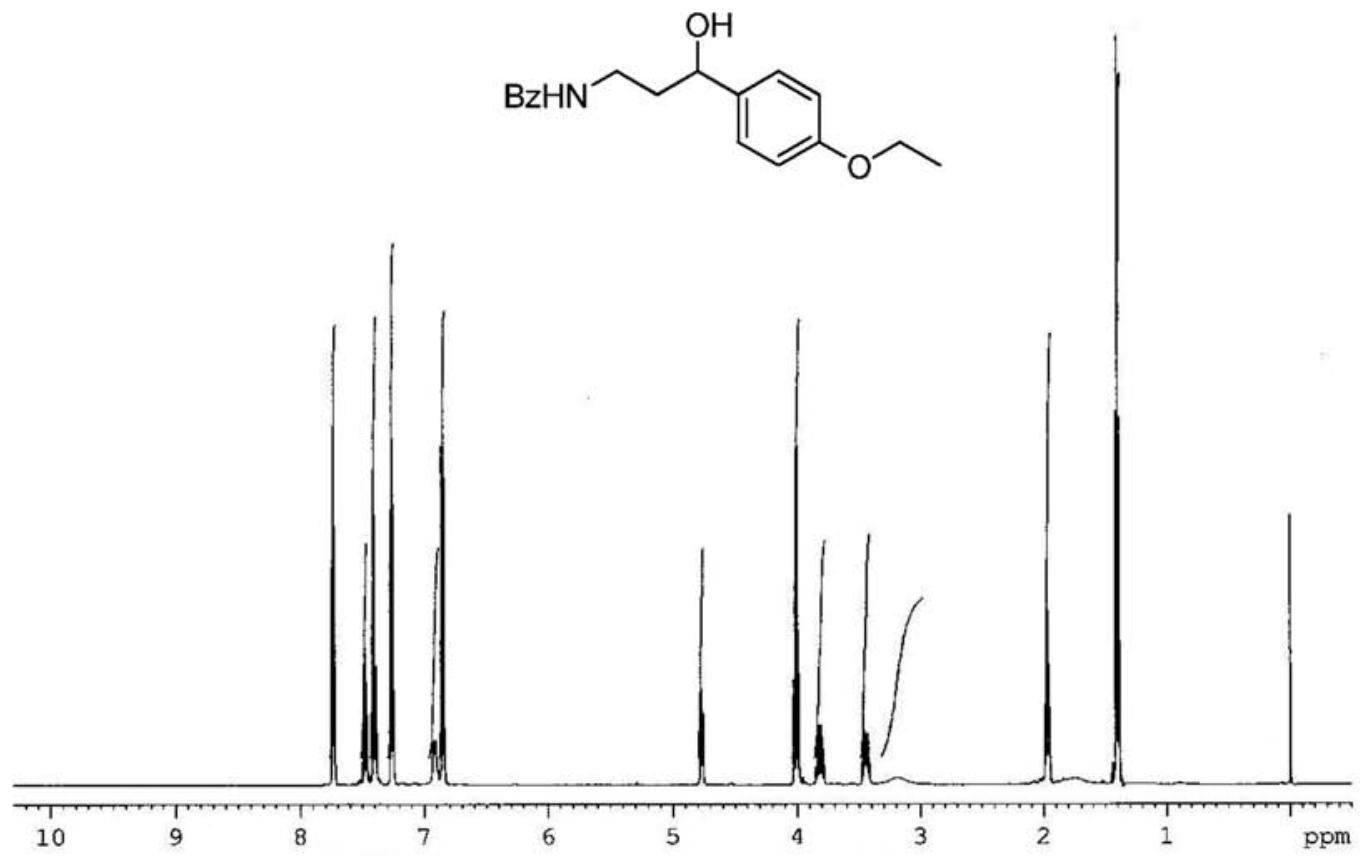

Figure S27. ${ }^{1} \mathrm{H}$ NMR $\left(500 \mathrm{MHz}, \mathrm{CDCl}_{3}\right)$ spectrum of $2 \mathbf{e}$.

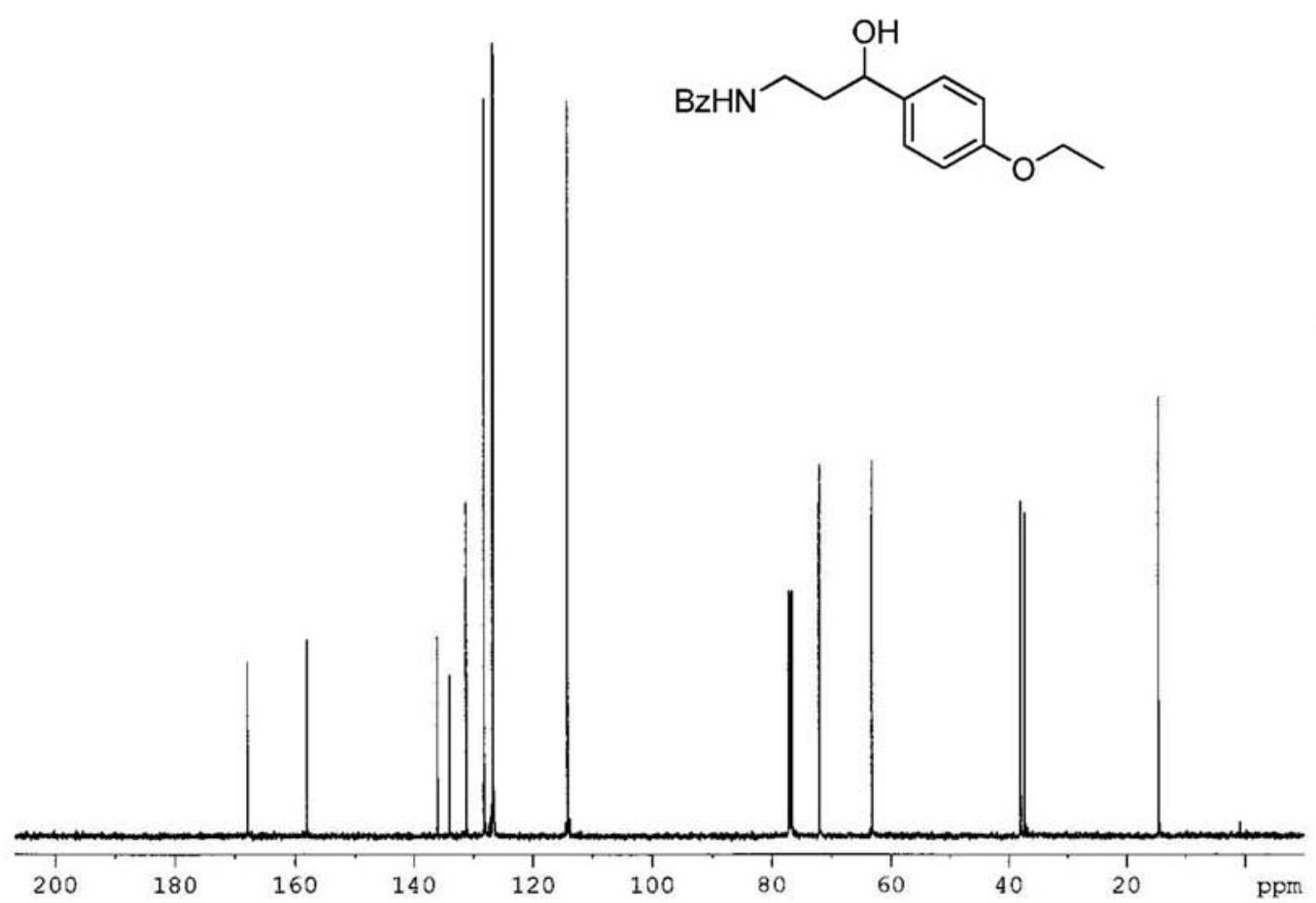

Figure S28. ${ }^{13} \mathrm{C}$ NMR (125 MHz, $\mathrm{CDCl}_{3}$ ) spectrum of $2 \mathbf{e}$. 


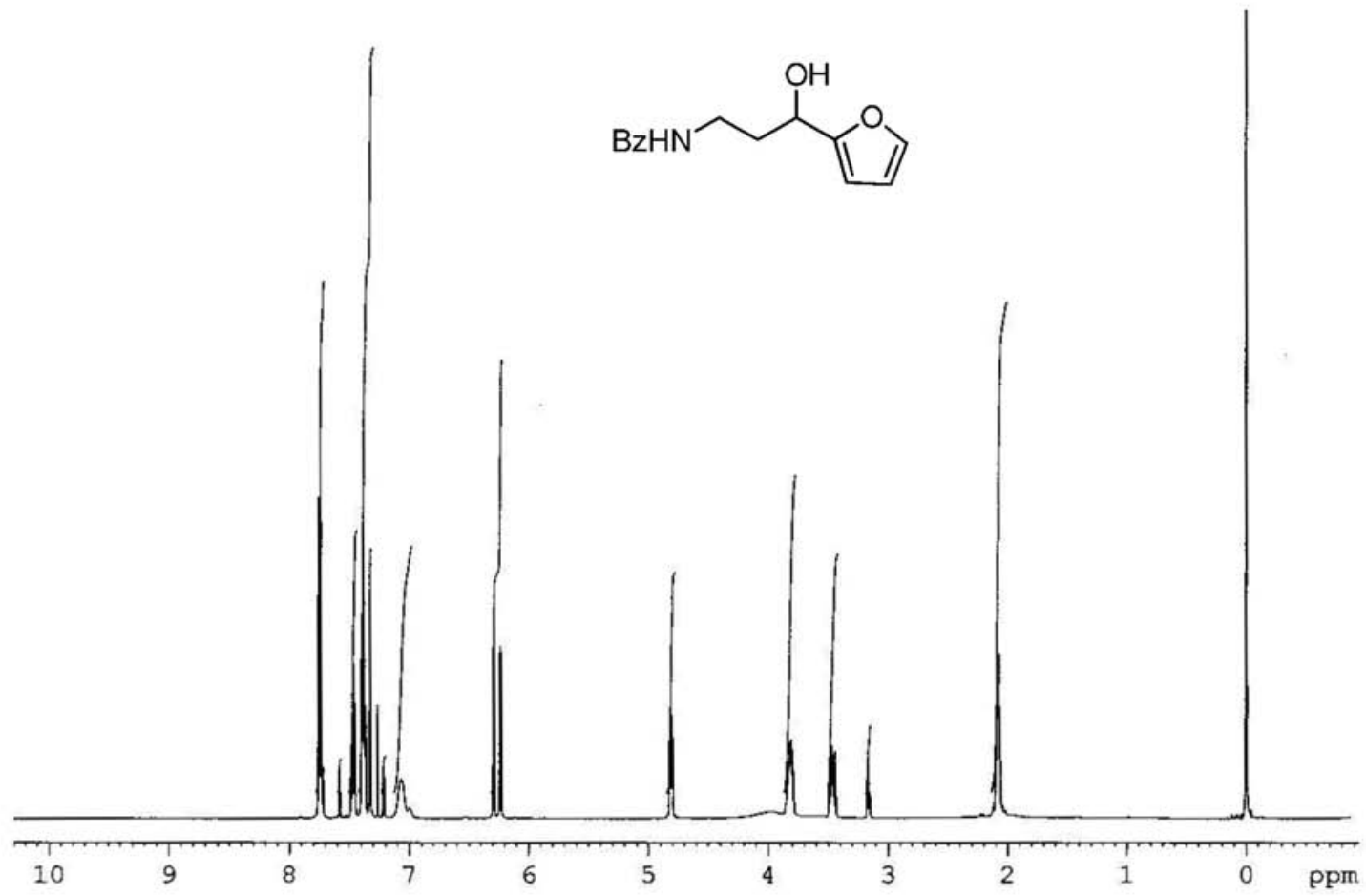

Figure S29. ${ }^{1} \mathrm{H}$ NMR $\left(500 \mathrm{MHz}, \mathrm{CDCl}_{3}\right)$ spectrum of $2 \mathbf{f}$.

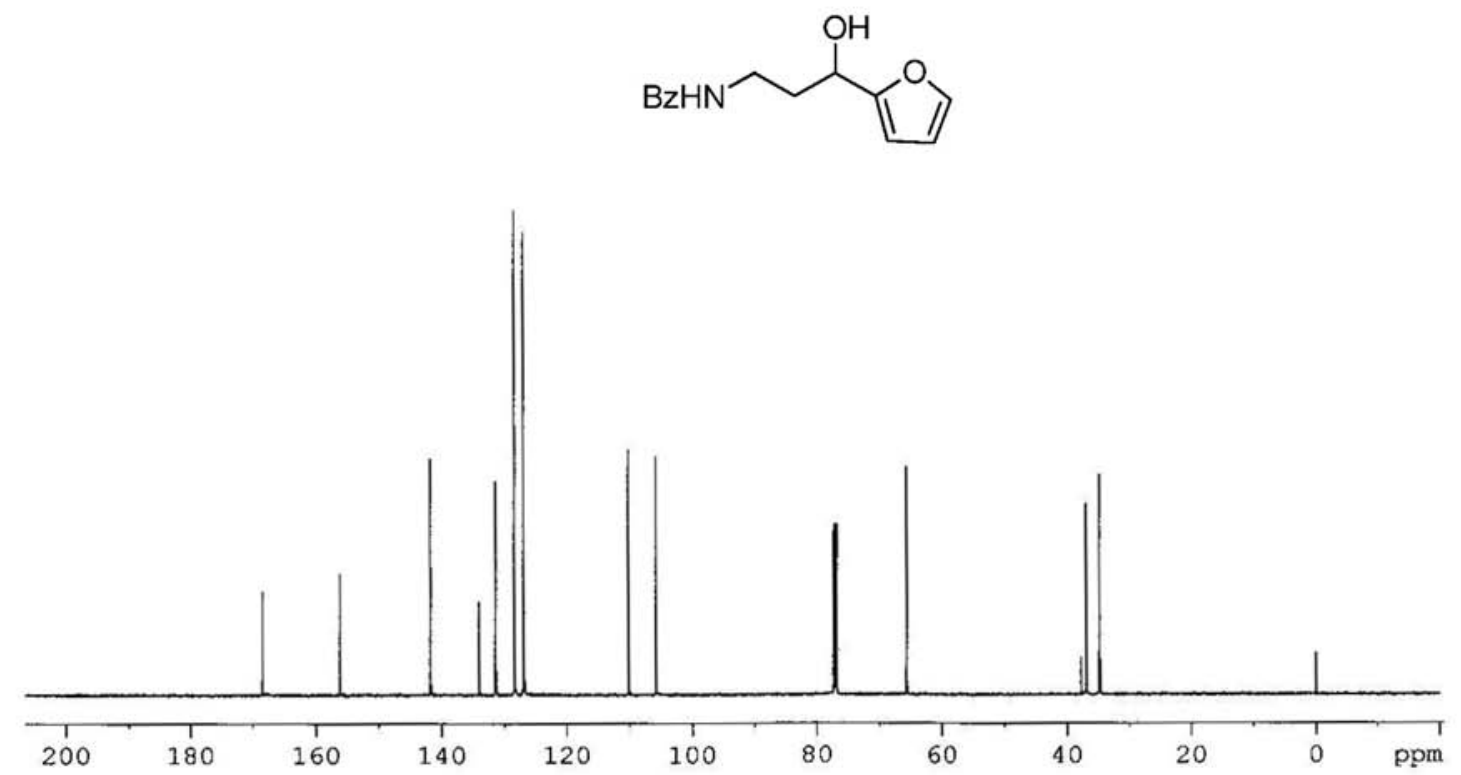

Figure S30. ${ }^{13} \mathrm{C}$ NMR $\left(125 \mathrm{MHz}, \mathrm{CDCl}_{3}\right)$ spectrum of $2 \mathbf{f}$. 


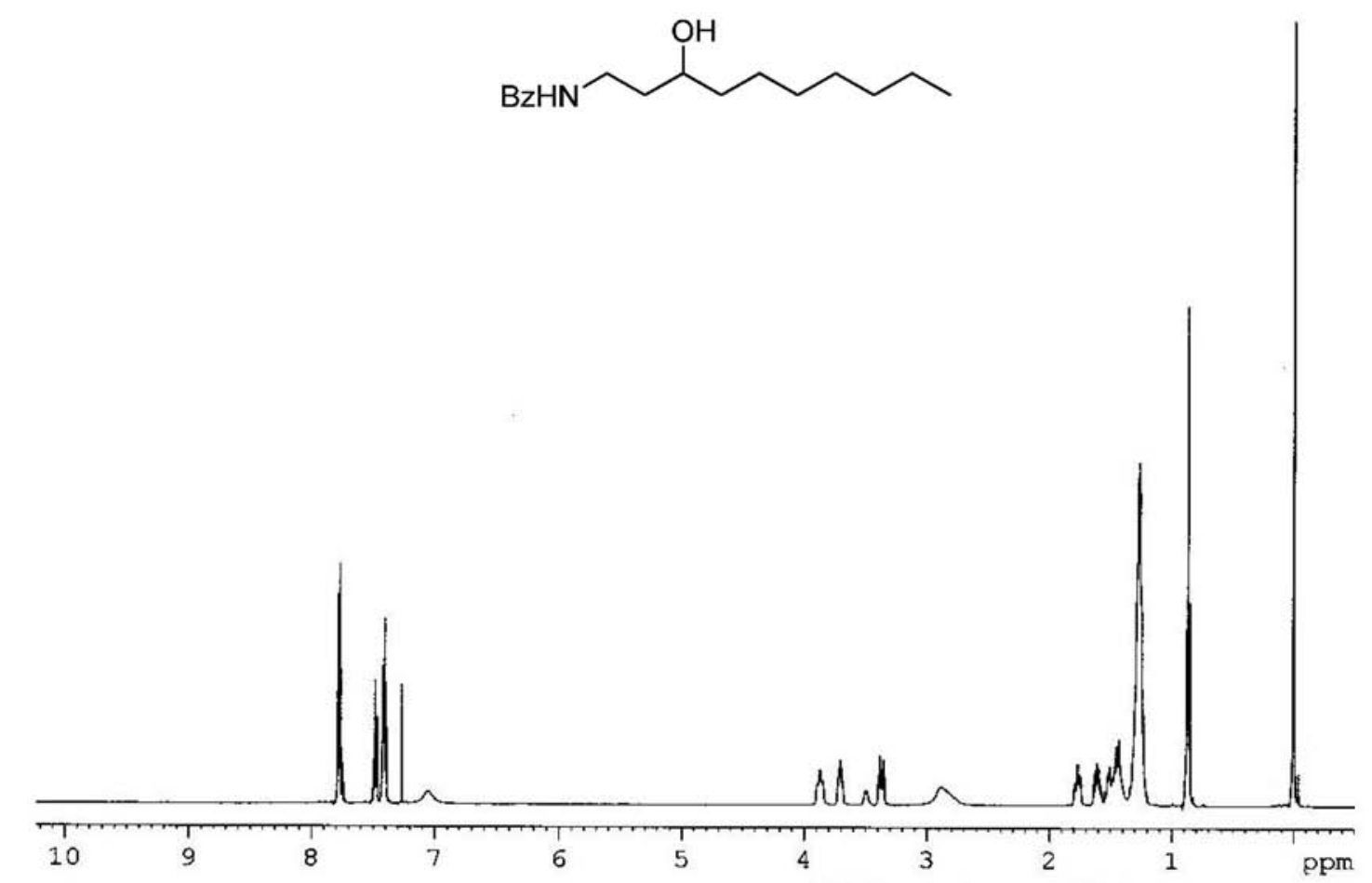

Figure S31. ${ }^{1} \mathrm{H} \mathrm{NMR}\left(500 \mathrm{MHz}, \mathrm{CDCl}_{3}\right)$ spectrum of $\mathbf{2 g}$.<smiles>CCCCCCCC(O)CCNC(=O)c1ccccc1</smiles>

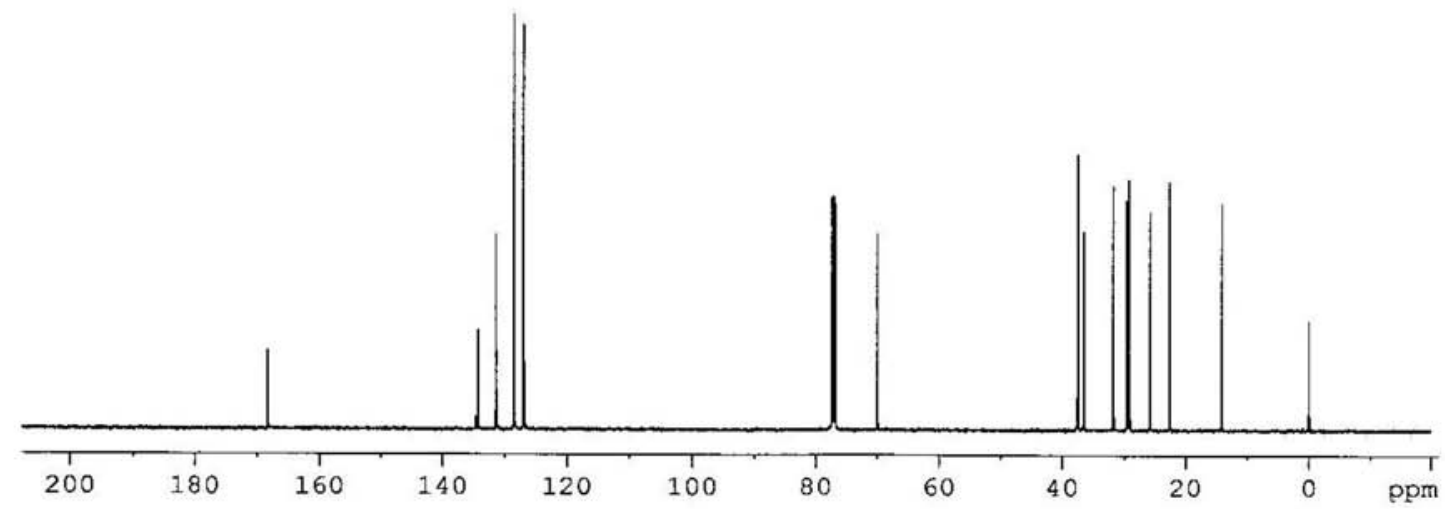

Figure S32. ${ }^{13} \mathrm{C}$ NMR $\left(125 \mathrm{MHz}, \mathrm{CDCl}_{3}\right)$ spectrum of $\mathbf{2 g}$. 


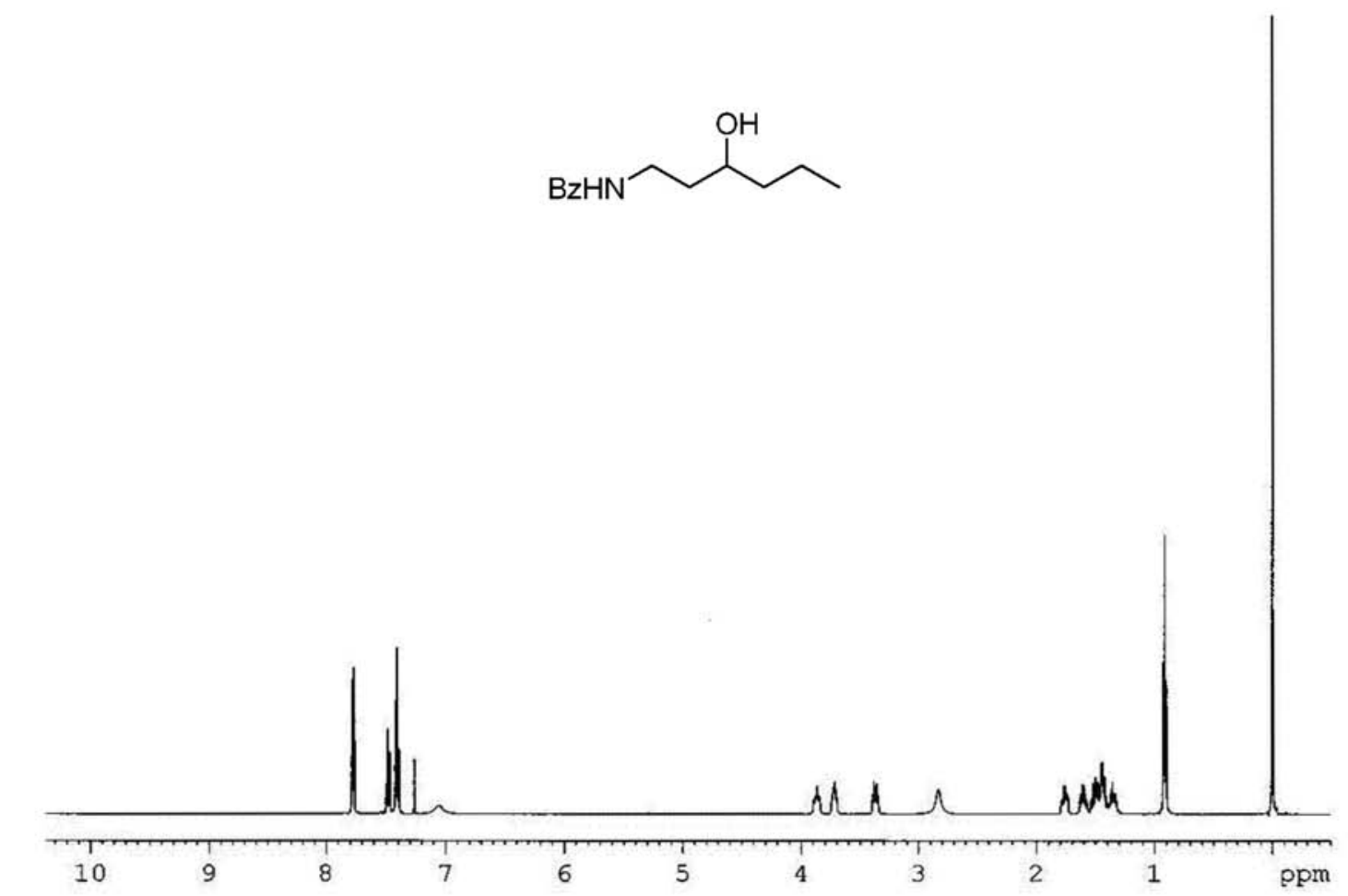

Figure S33. ${ }^{1} \mathrm{H}$ NMR $\left(500 \mathrm{MHz}, \mathrm{CDCl}_{3}\right)$ spectrum of $\mathbf{2 h}$.
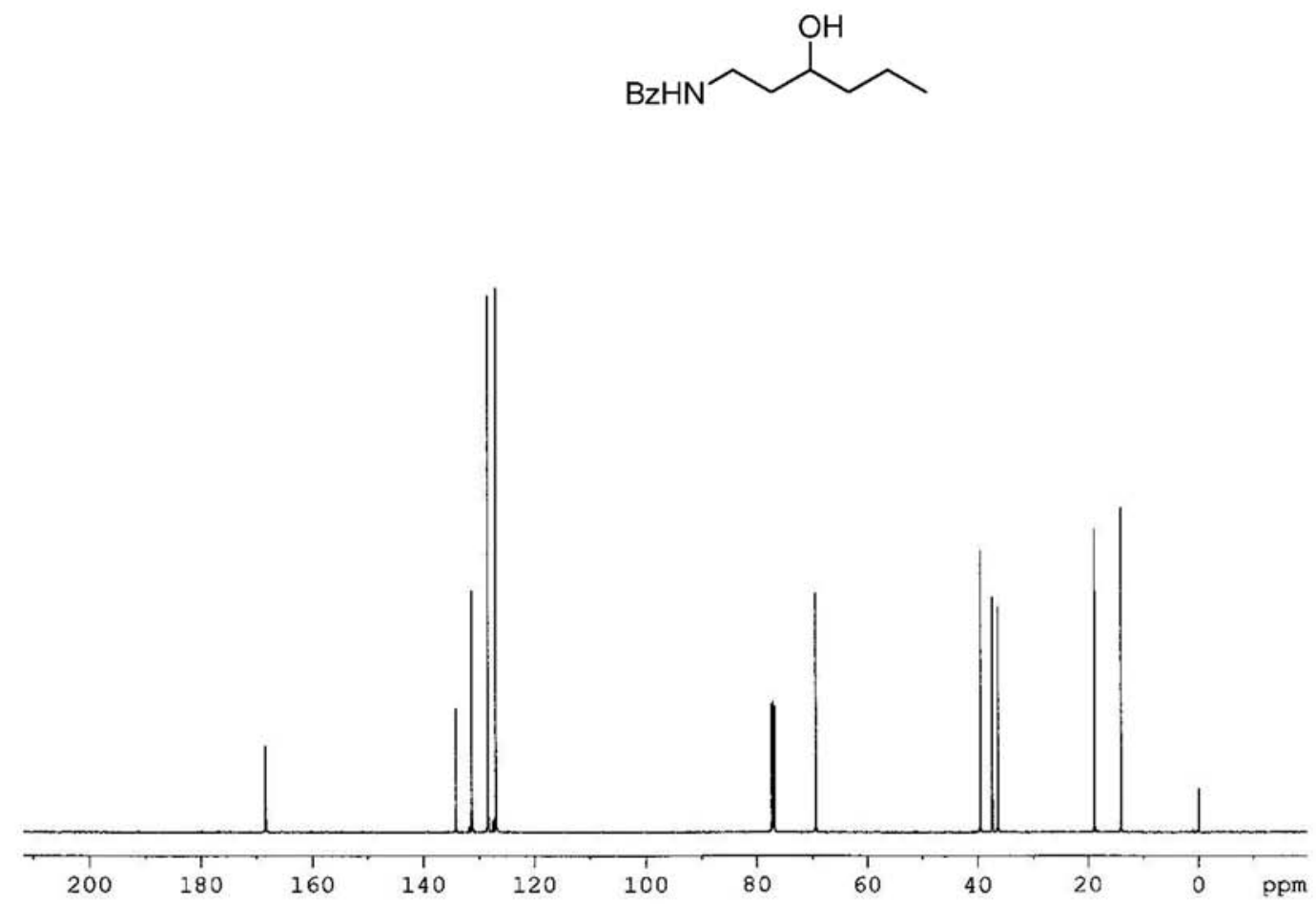

Figure S34. ${ }^{13} \mathrm{C}$ NMR $\left(125 \mathrm{MHz}, \mathrm{CDCl}_{3}\right)$ spectrum of $\mathbf{2 h}$. 


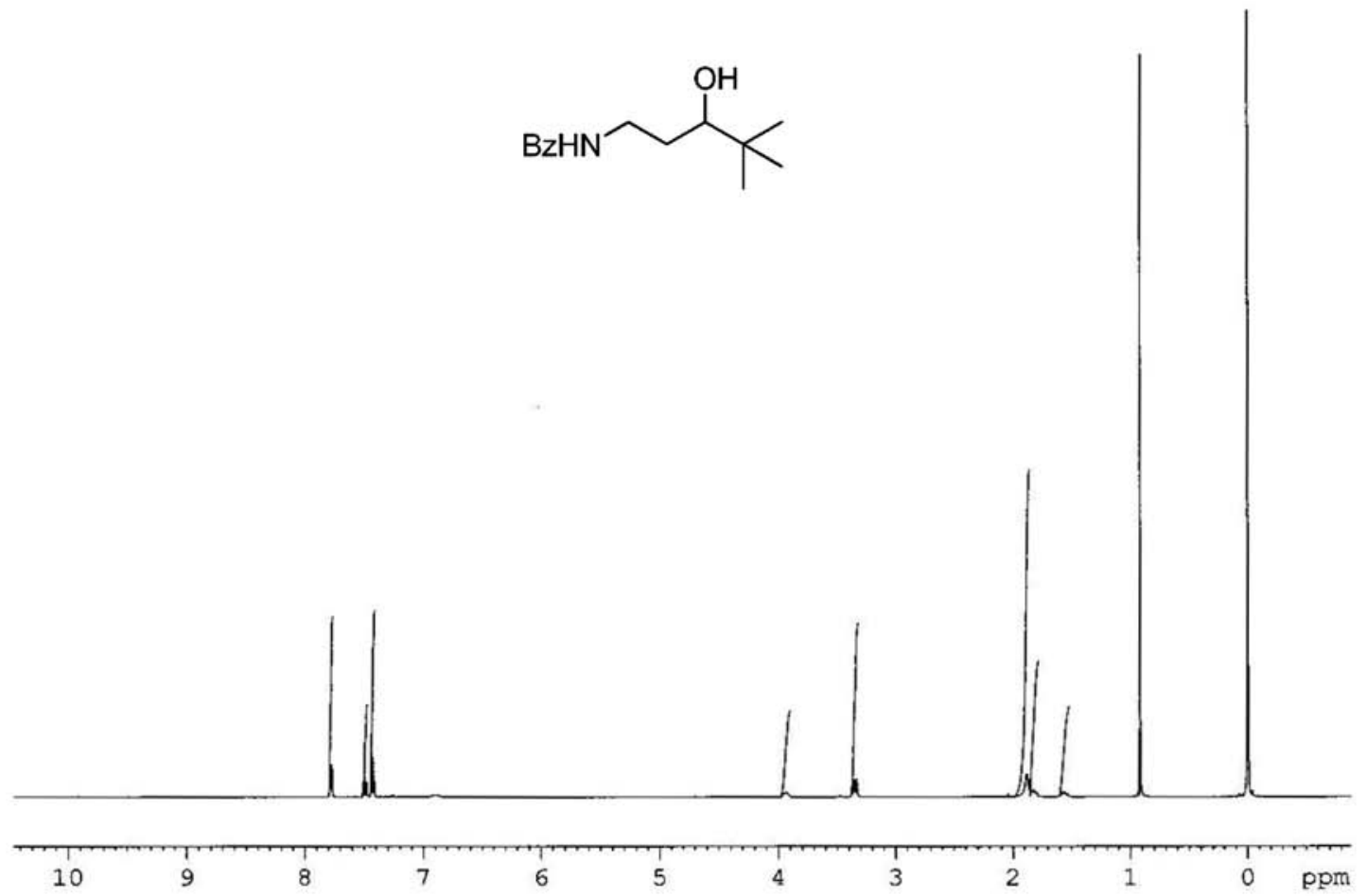

Figure S35. ${ }^{1} \mathrm{H}$ NMR $\left(500 \mathrm{MHz}, \mathrm{CDCl}_{3}\right)$ spectrum of $2 \mathbf{i}$.

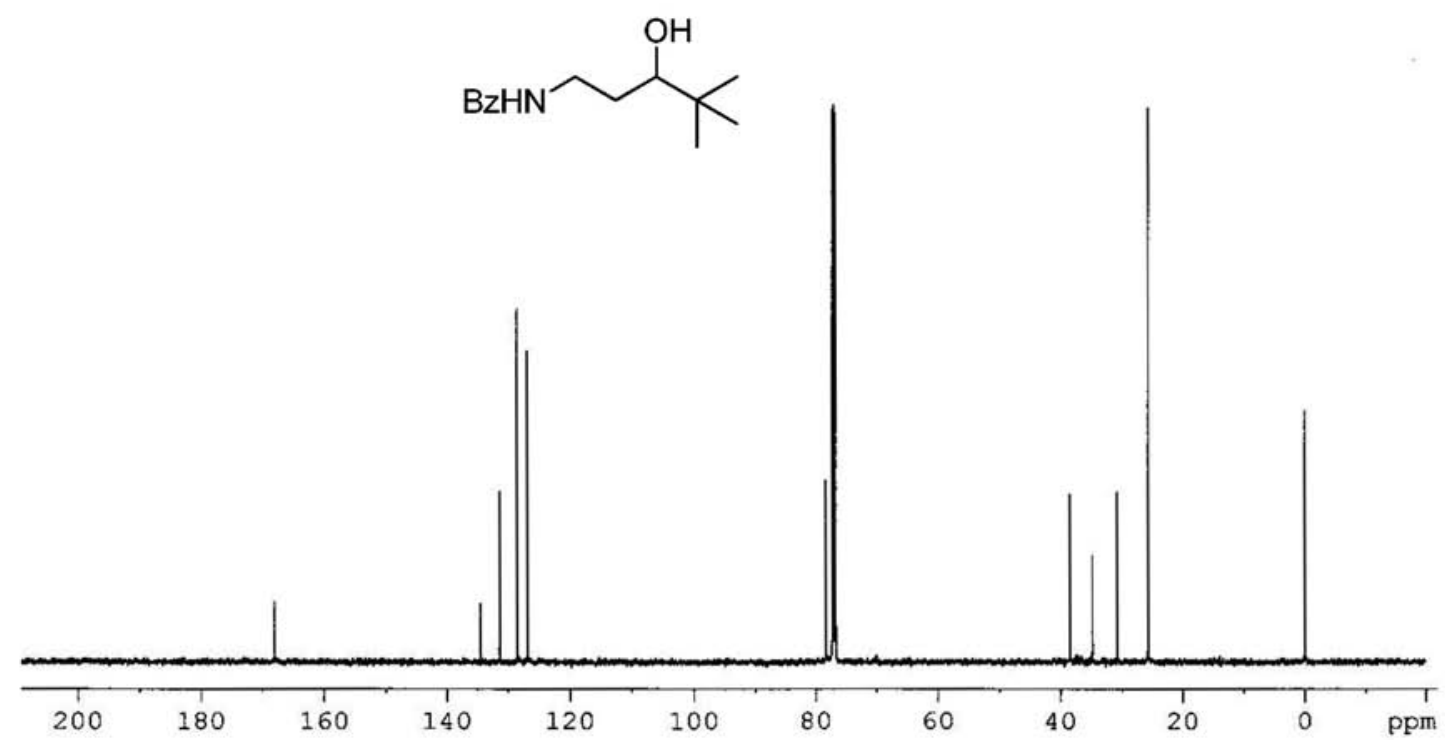

Figure S36. ${ }^{13} \mathrm{C}$ NMR (125 MHz, $\mathrm{CDCl}_{3}$ ) spectrum of 2 i. 


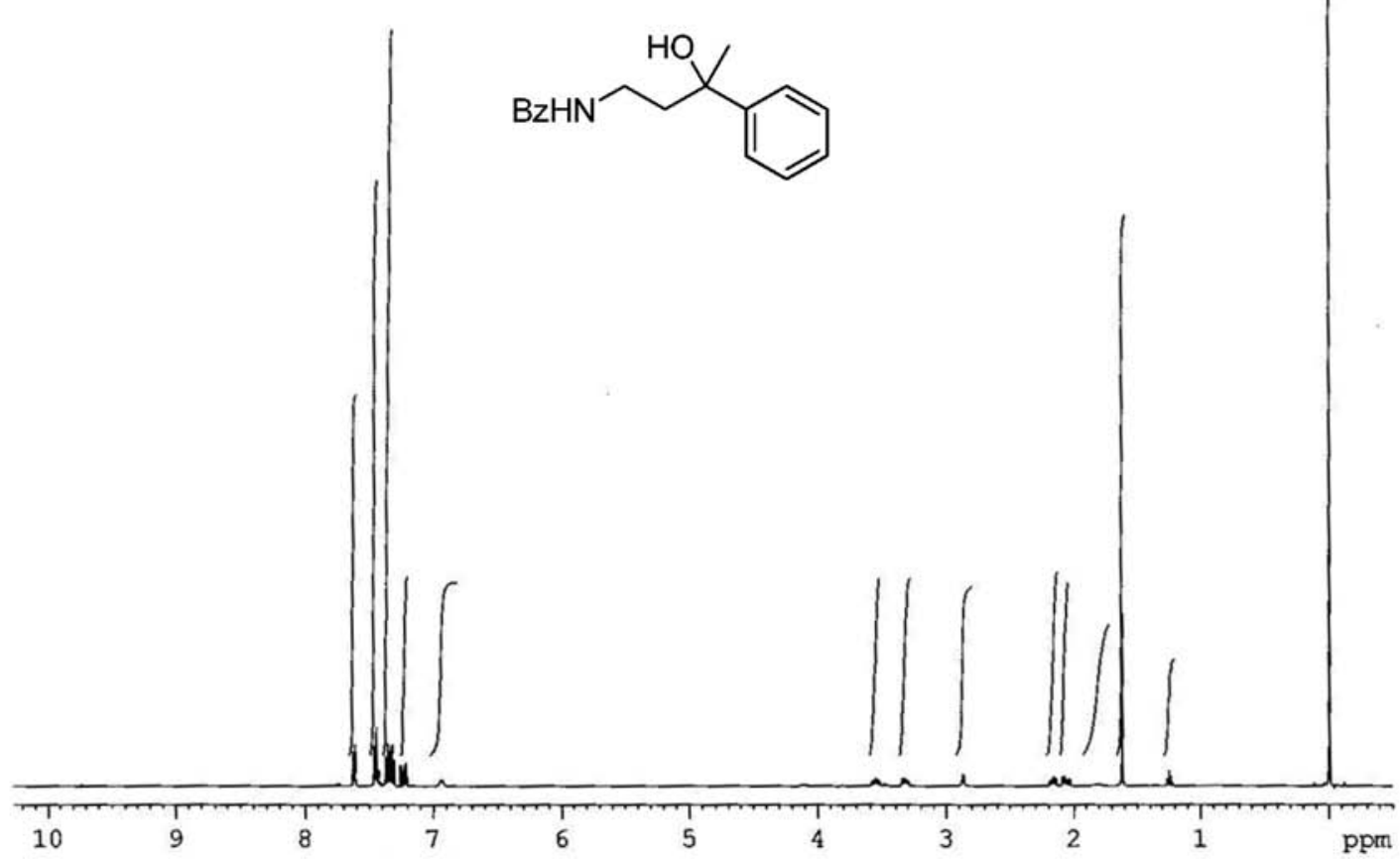

Figure S37. ${ }^{1} \mathrm{H}$ NMR $\left(500 \mathrm{MHz}, \mathrm{CDCl}_{3}\right)$ spectrum of $\mathbf{2 j}$.
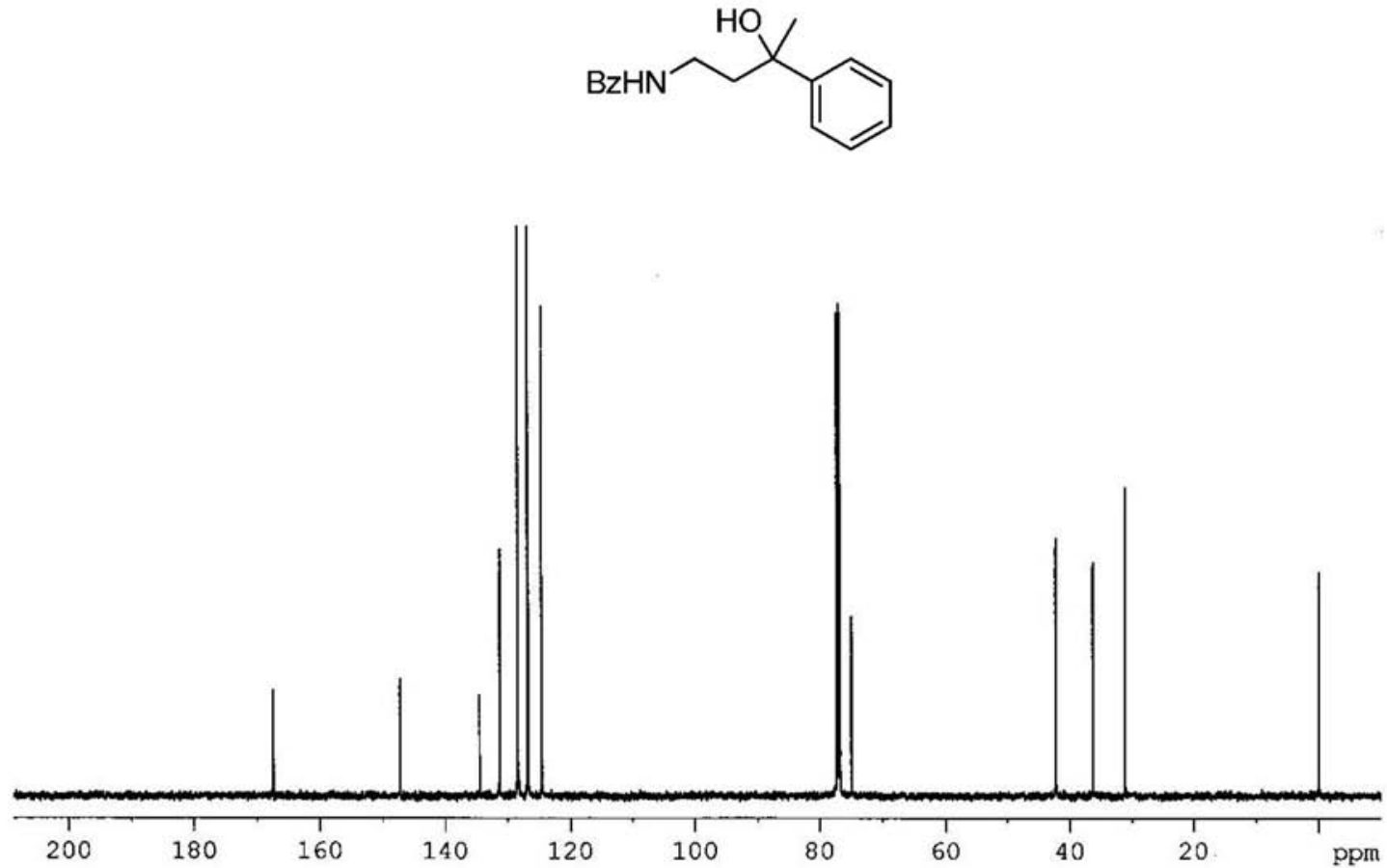

Figure S38. ${ }^{13} \mathrm{C}$ NMR $\left(125 \mathrm{MHz}, \mathrm{CDCl}_{3}\right)$ spectrum of $\mathbf{2 j}$. 


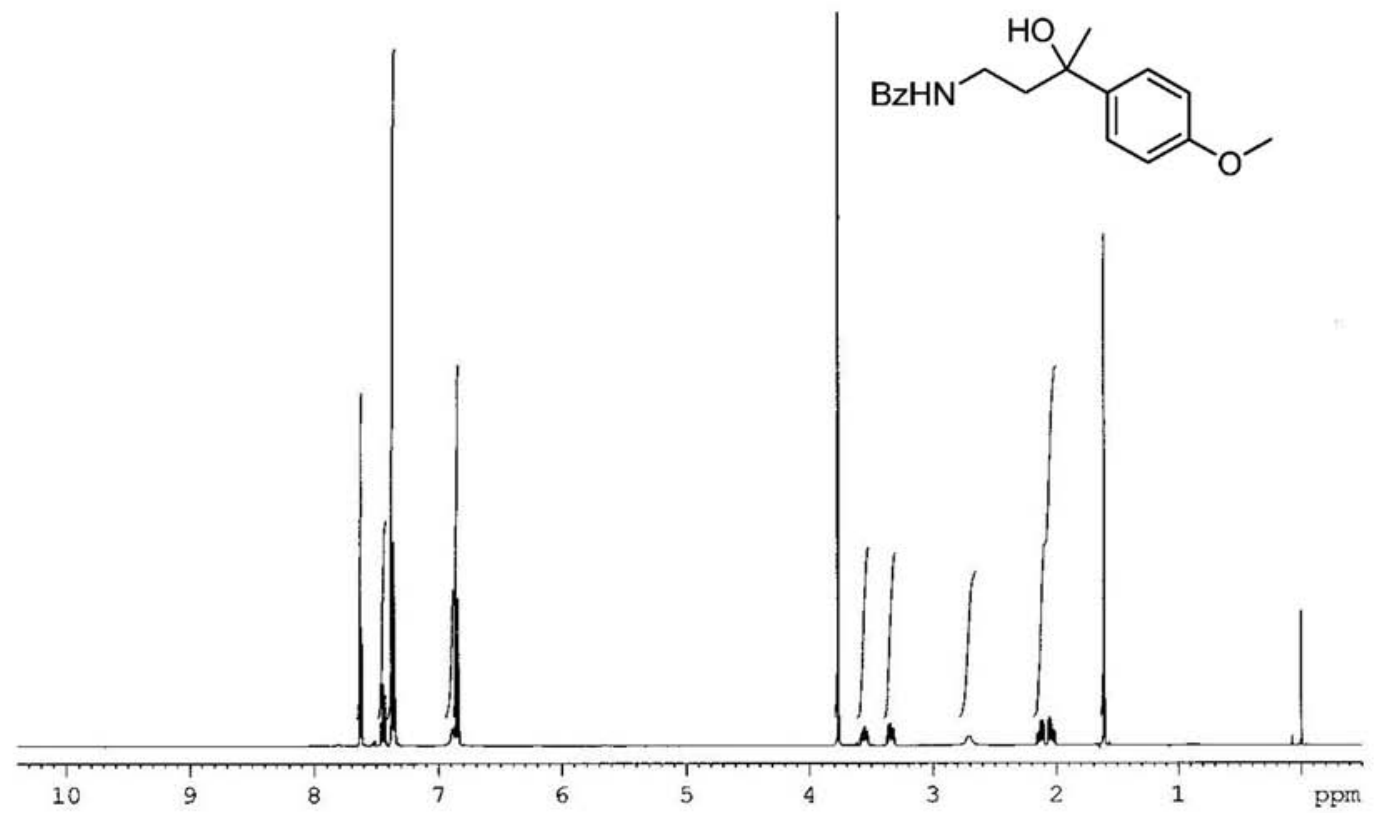

Figure S39. ' $\mathrm{H}$ NMR $\left(500 \mathrm{MHz}, \mathrm{CDCl}_{3}\right)$ spectrum of $\mathbf{2 k}$.

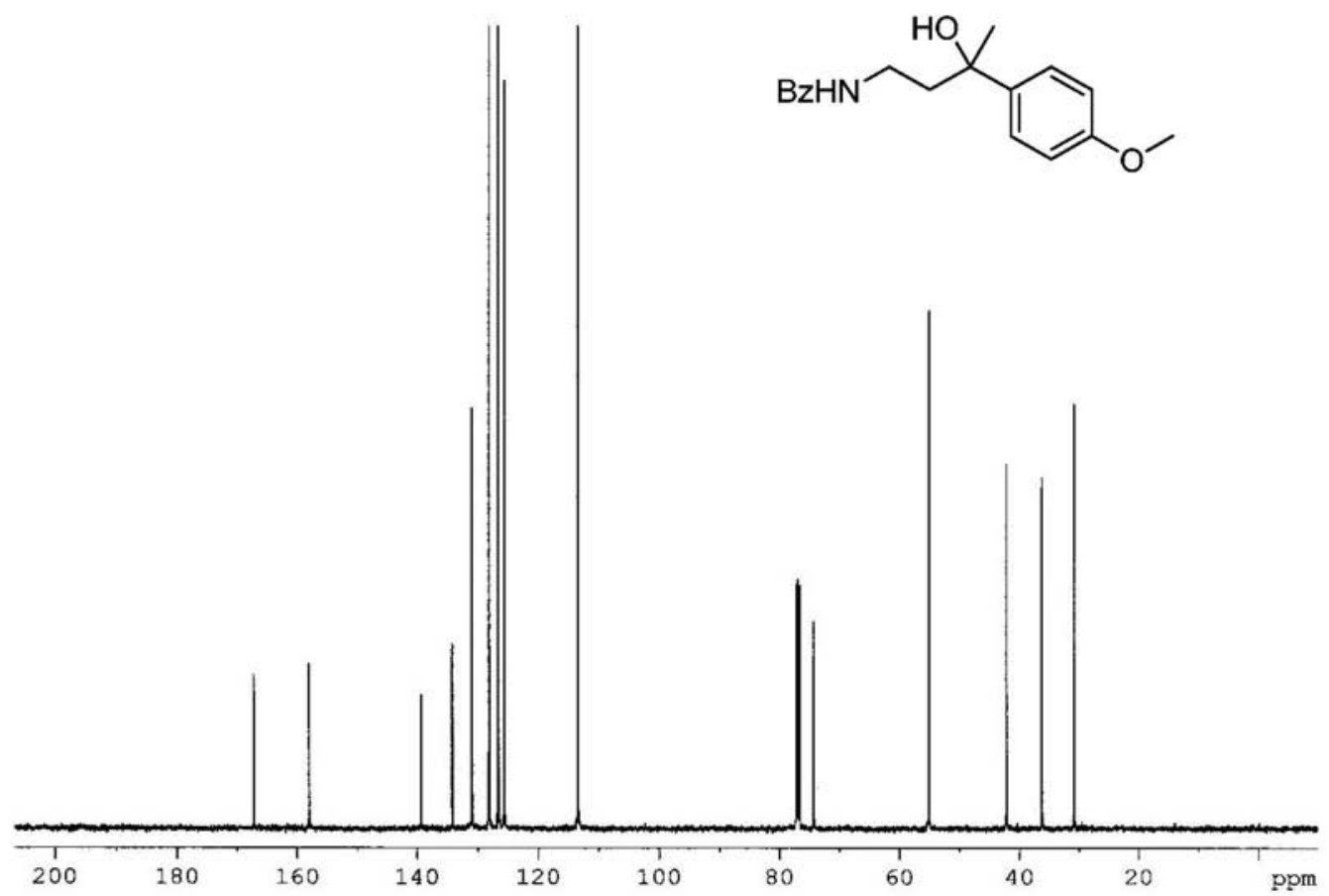

Figure S40. ${ }^{13} \mathrm{C}$ NMR $\left(125 \mathrm{MHz}, \mathrm{CDCl}_{3}\right)$ spectrum of $\mathbf{2 k}$. 


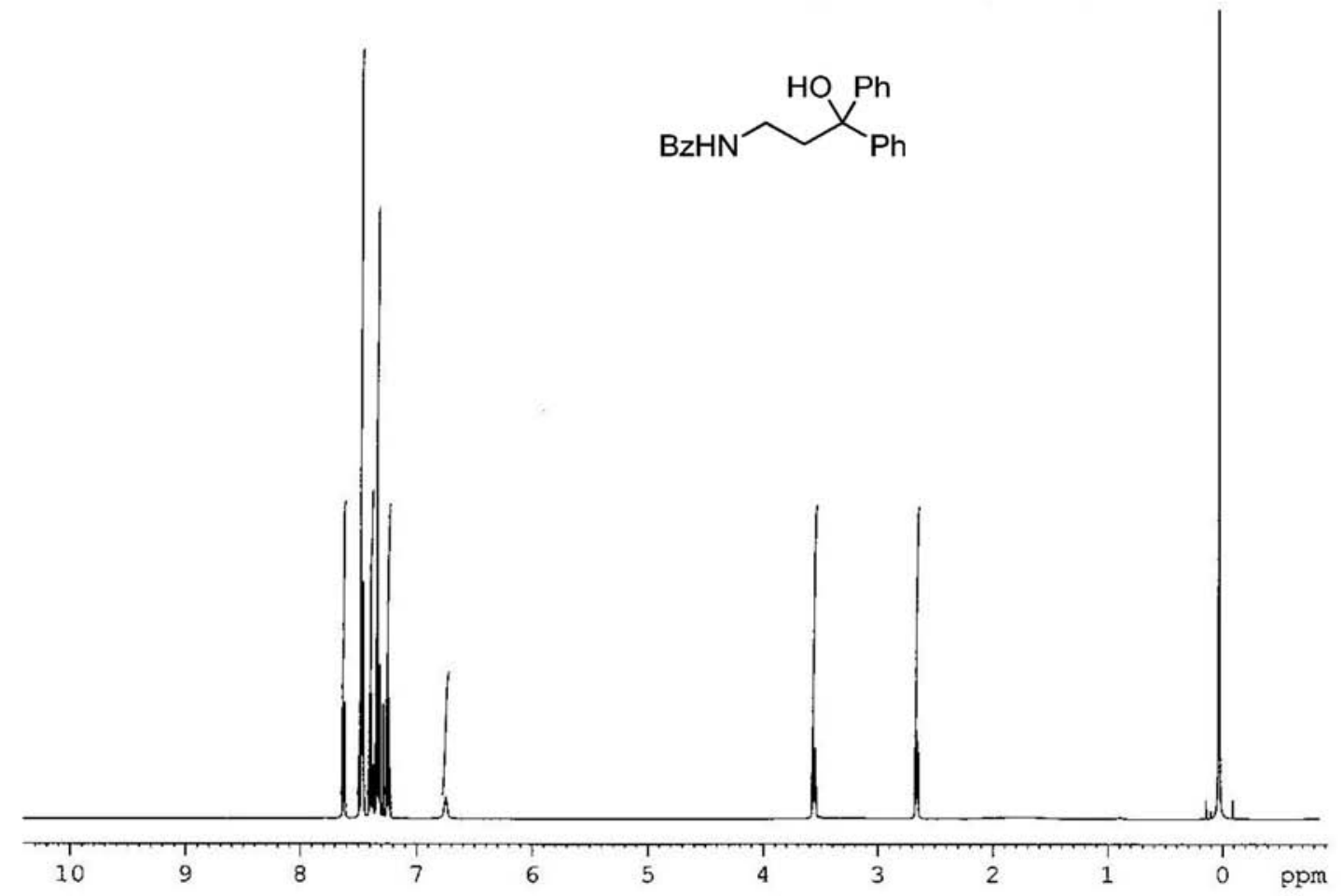

Figure S41. ${ }^{1} \mathrm{H}$ NMR $\left(500 \mathrm{MHz}, \mathrm{CDCl}_{3}\right)$ spectrum of $\mathbf{2 l}$.

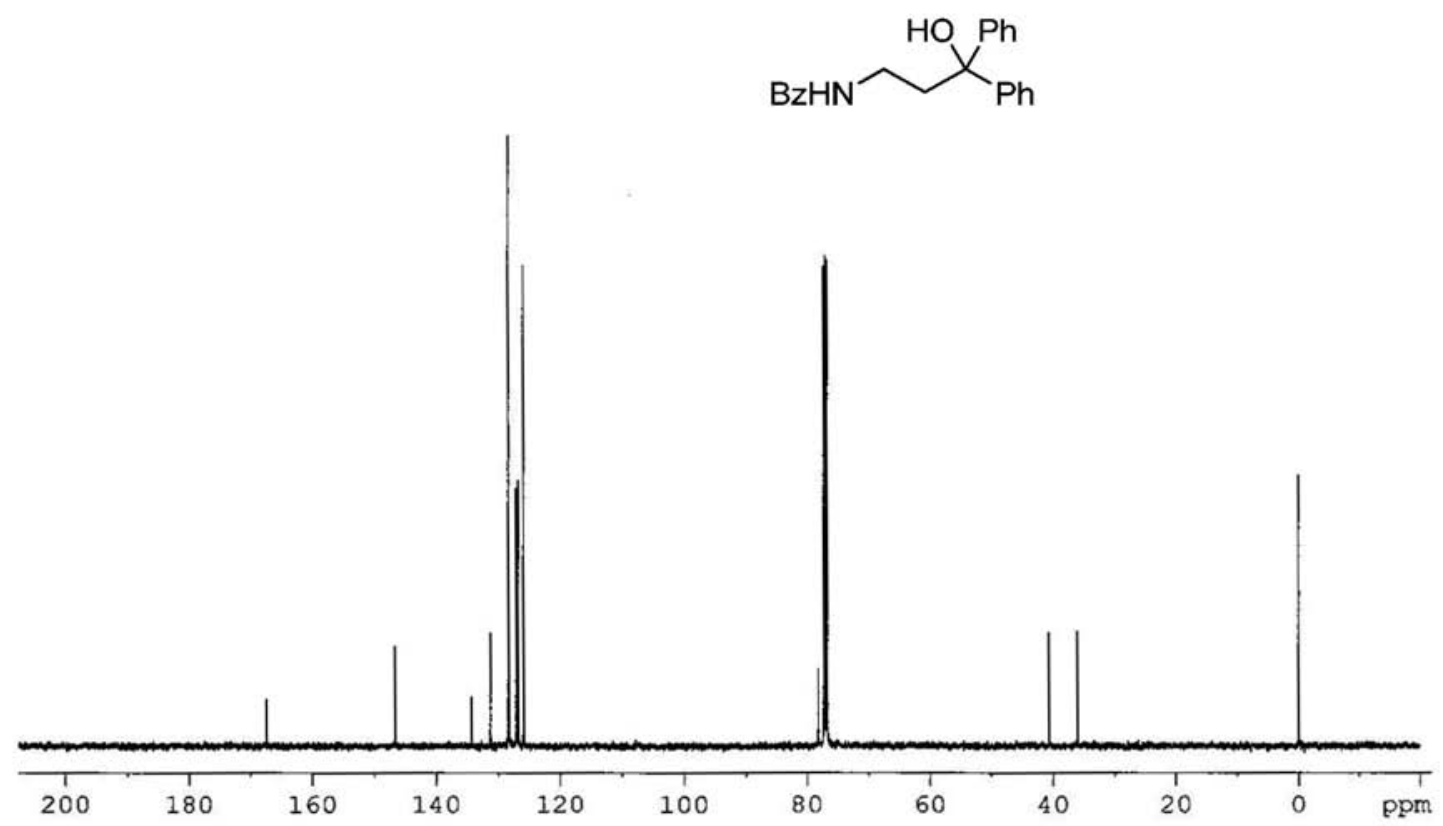

Figure S42. ${ }^{13} \mathrm{C}$ NMR $\left(125 \mathrm{MHz}, \mathrm{CDCl}_{3}\right)$ spectrum of $2 \mathbf{2}$. 


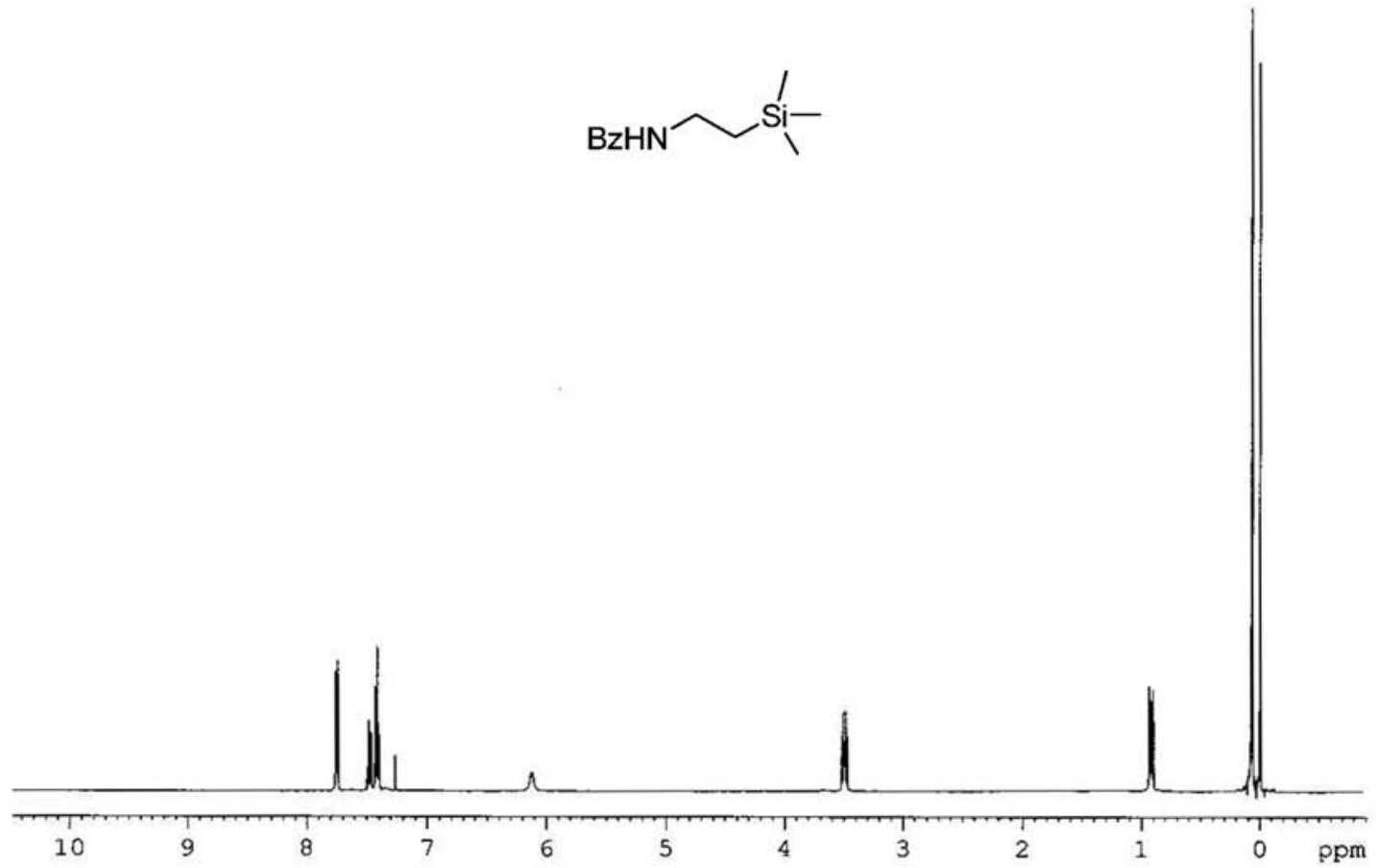

Figure S43. ${ }^{1} \mathrm{H}$ NMR $\left(500 \mathrm{MHz}, \mathrm{CDCl}_{3}\right)$ spectrum of $\mathbf{2} \mathbf{m}$.

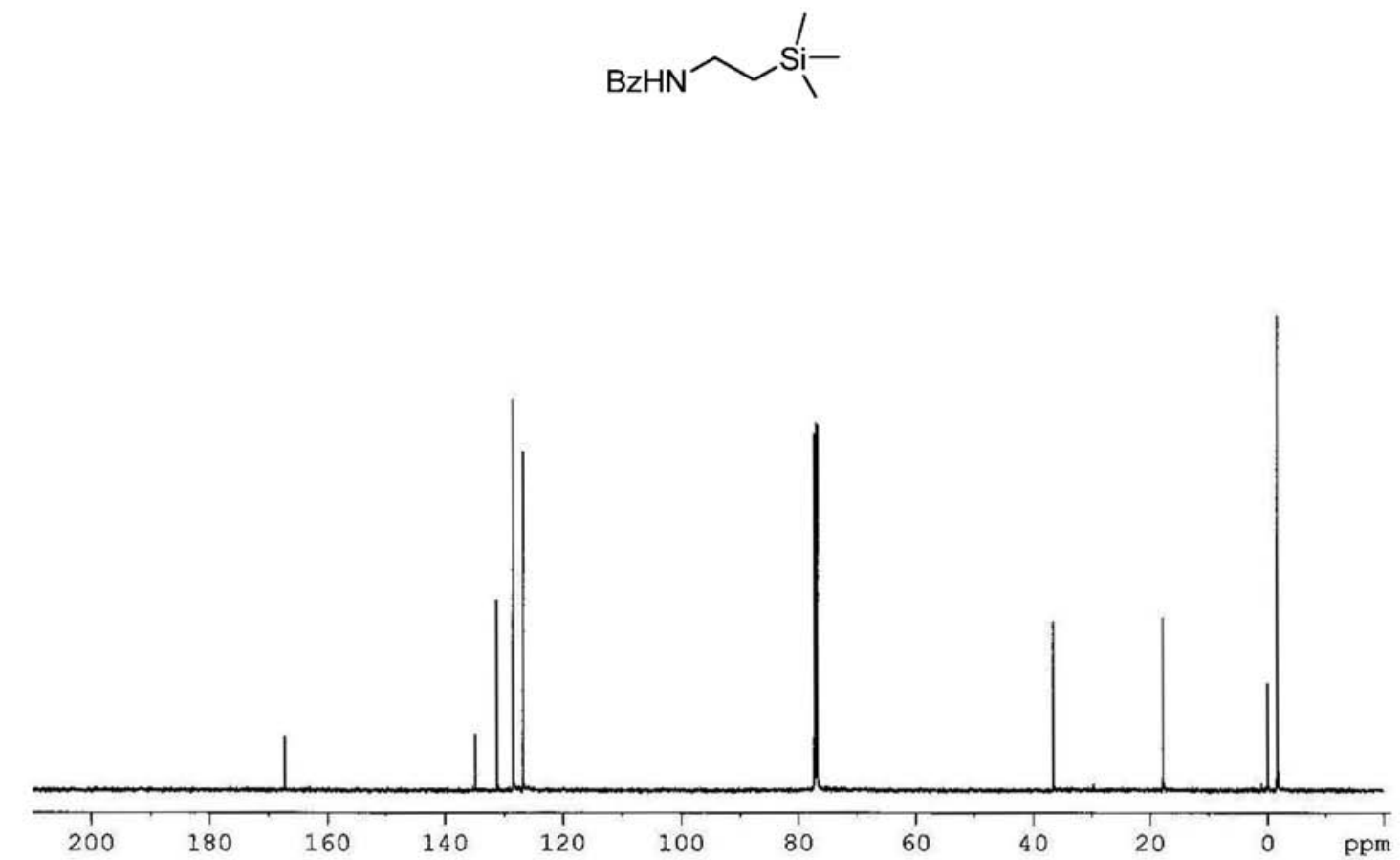

Figure S44. ${ }^{13} \mathrm{C}$ NMR $\left(125 \mathrm{MHz}, \mathrm{CDCl}_{3}\right)$ spectrum of $\mathbf{2 m}$. 


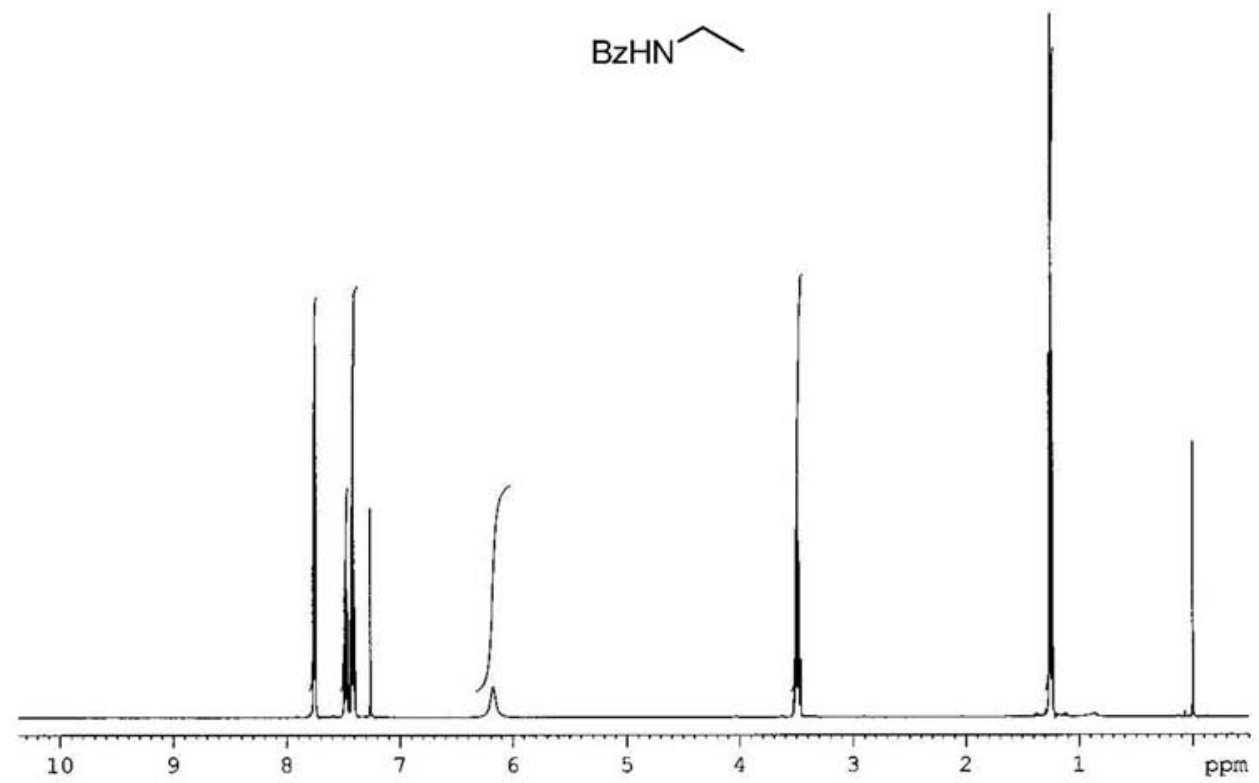

Figure $\mathbf{S 4 5} .{ }^{1} \mathrm{H} \mathrm{NMR}\left(500 \mathrm{MHz}, \mathrm{CDCl}_{3}\right)$ spectrum of $\mathbf{2 n}$.

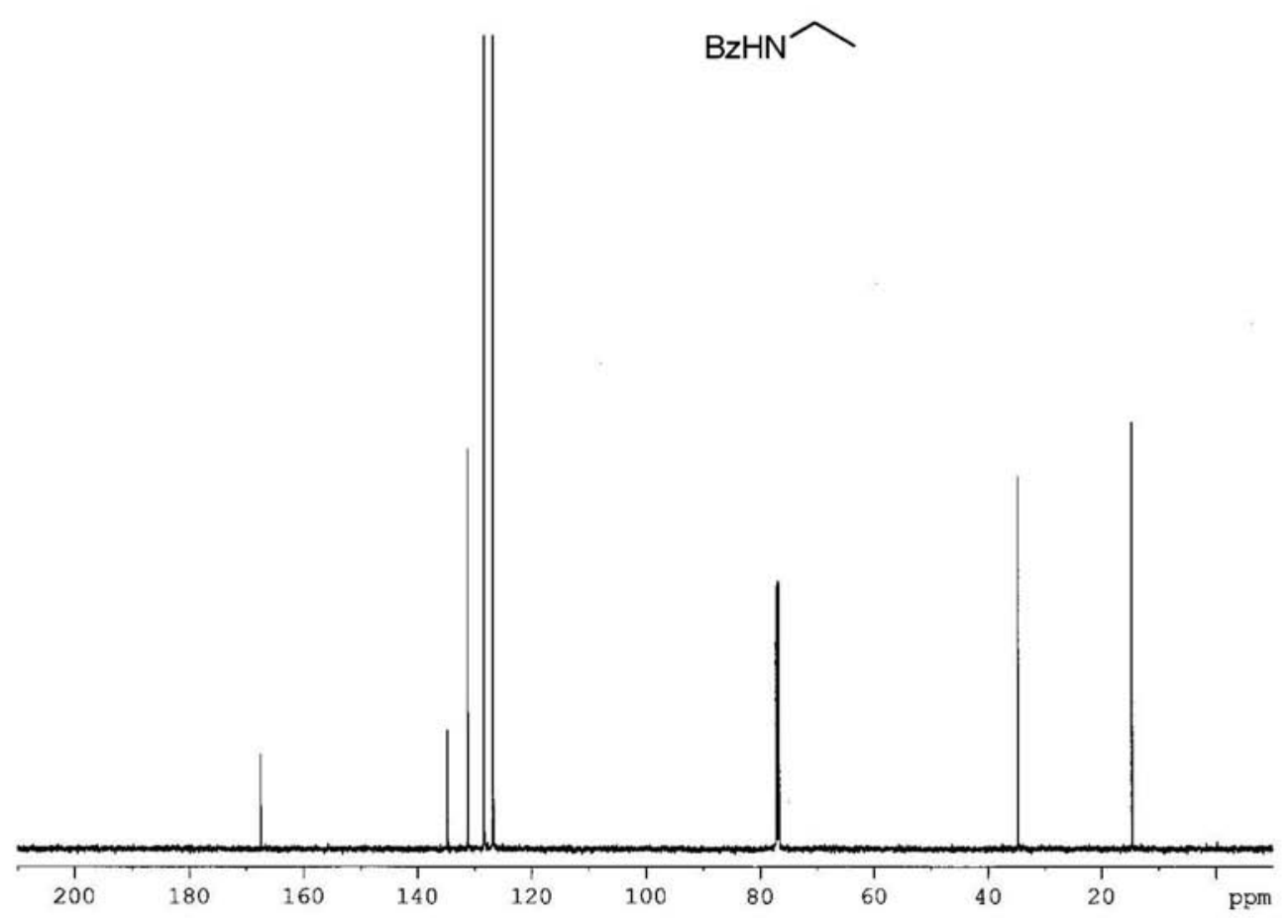

Figure S46. ${ }^{13} \mathrm{C}$ NMR $\left(125 \mathrm{MHz}, \mathrm{CDCl}_{3}\right)$ spectrum of $\mathbf{2 n}$. 


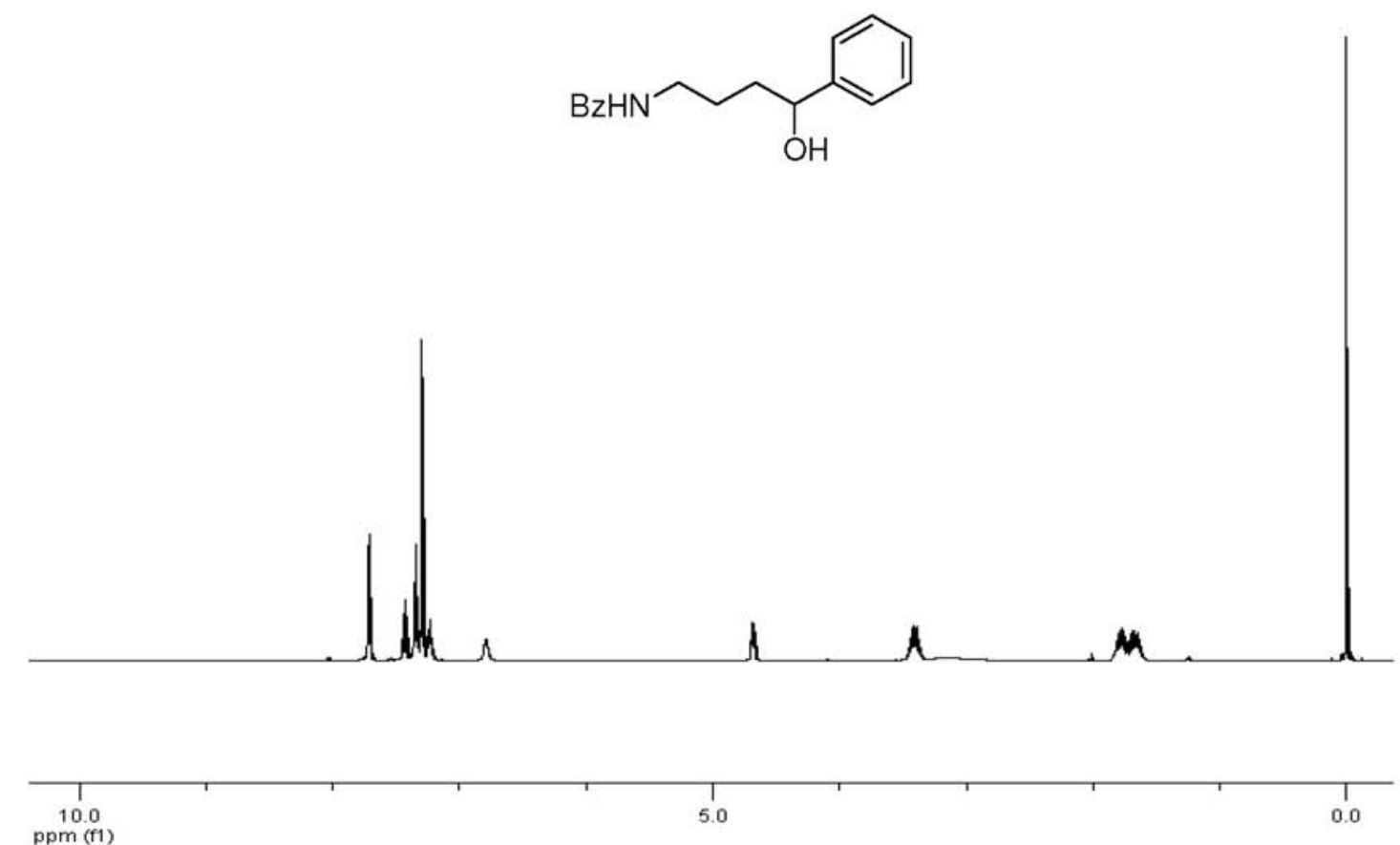

Figure S47. ${ }^{1} \mathrm{H}$ NMR $\left(500 \mathrm{MHz}, \mathrm{CDCl}_{3}\right)$ spectrum of 2 .
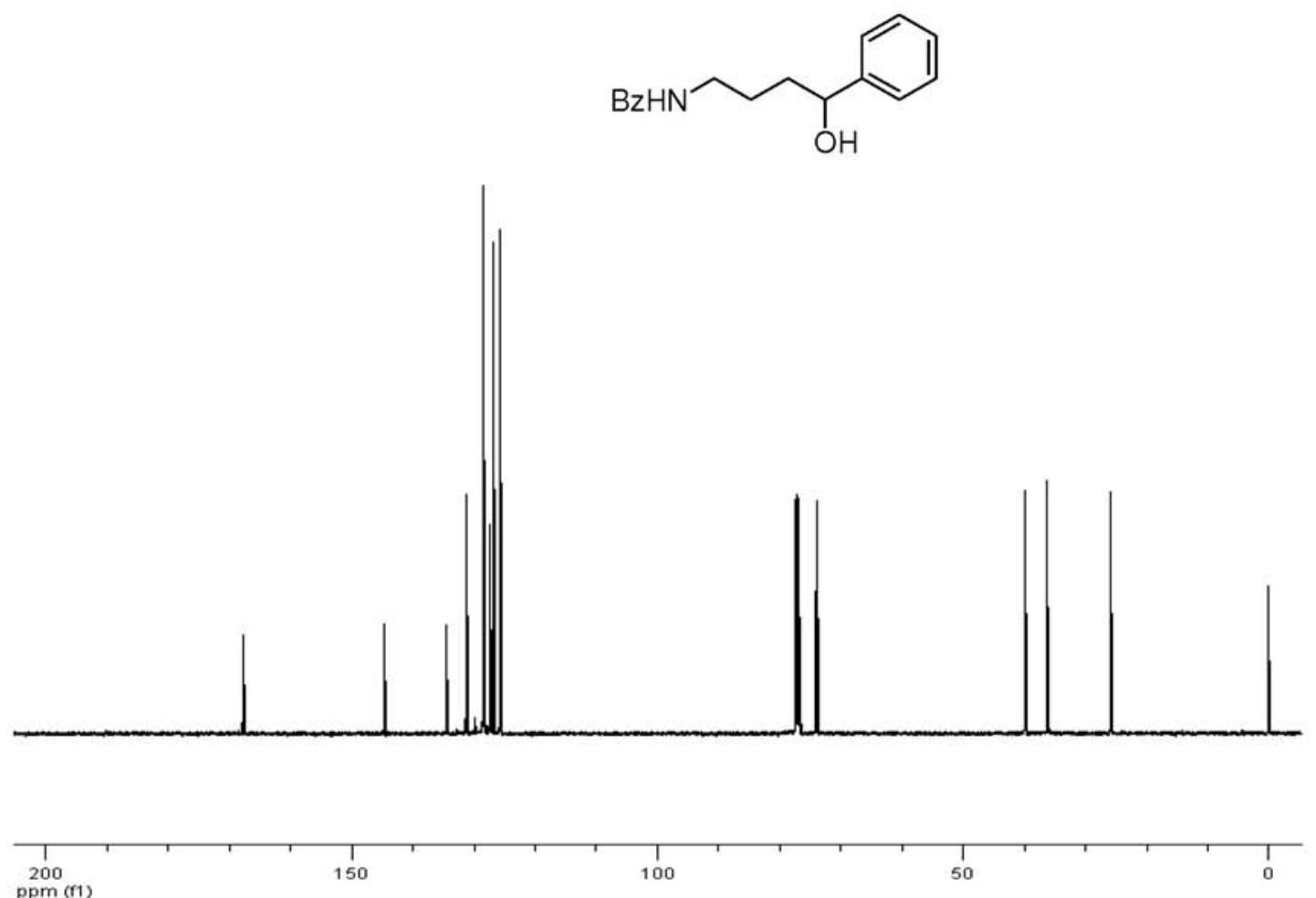

Figure S48. ${ }^{13} \mathrm{C}$ NMR $\left(125 \mathrm{MHz}, \mathrm{CDCl}_{3}\right)$ spectrum of 2 . 


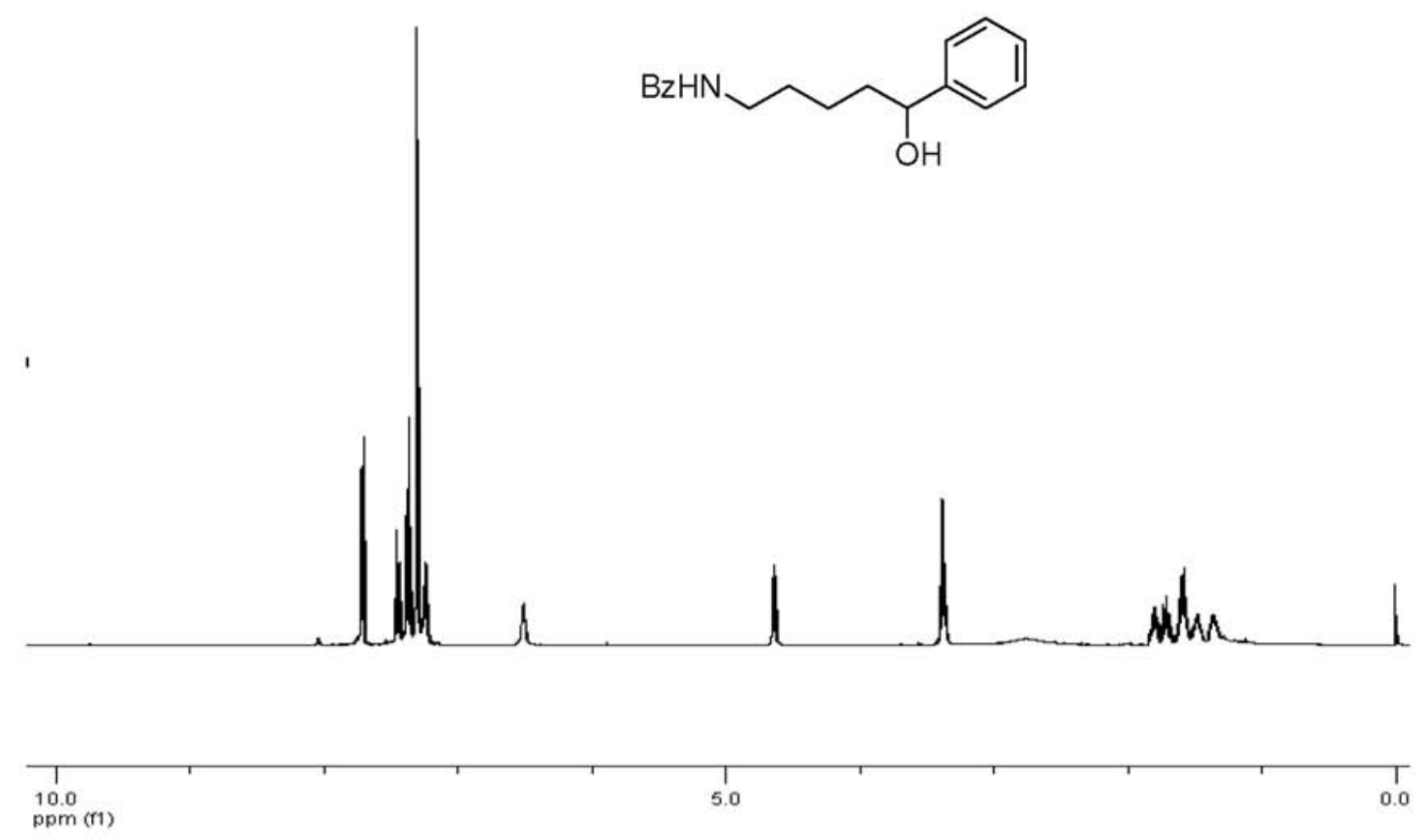

Figure S49. ${ }^{1} \mathrm{H}$ NMR $\left(500 \mathrm{MHz}, \mathrm{CDCl}_{3}\right)$ spectrum of $\mathbf{2 p}$.

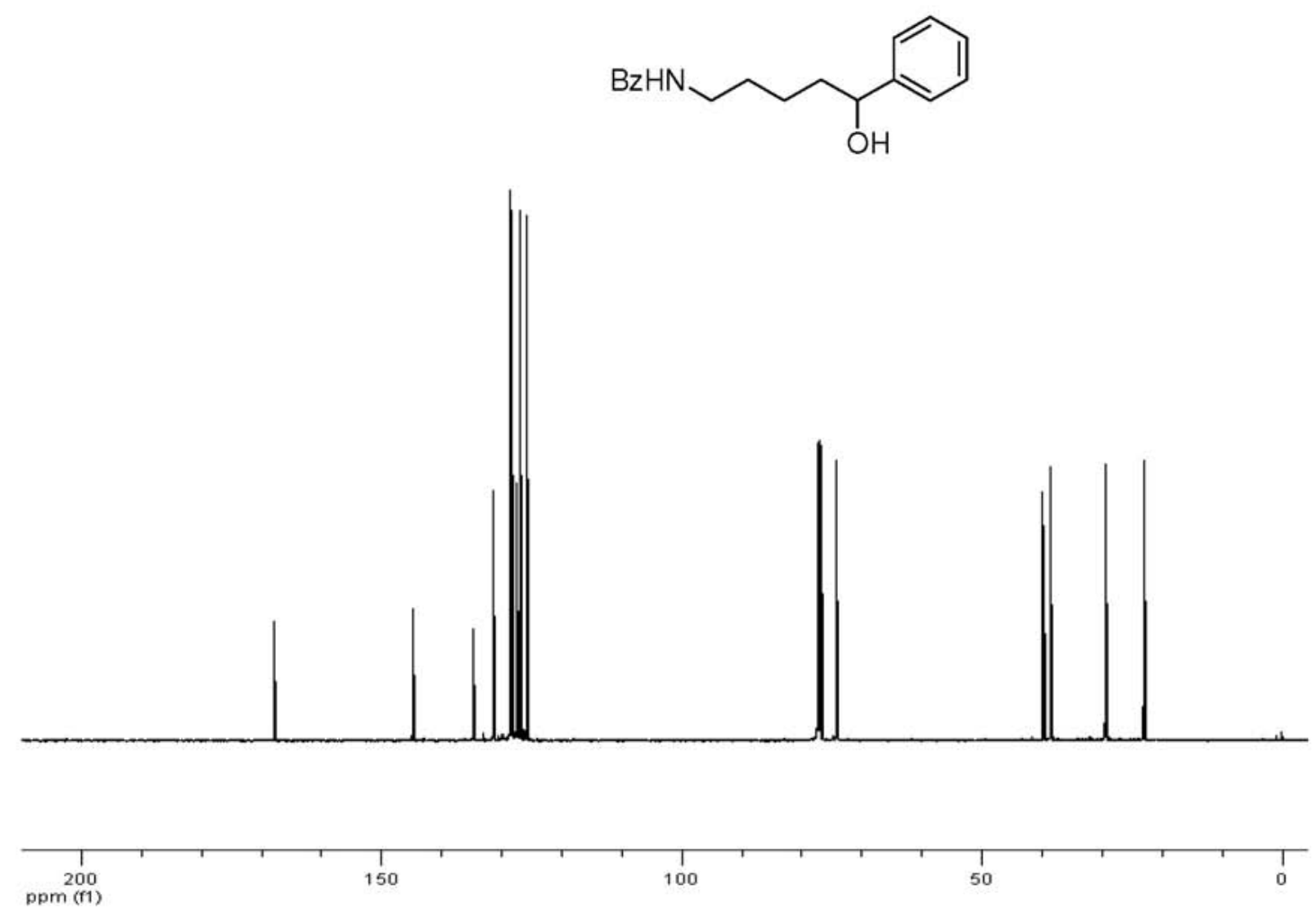

Figure S50. ${ }^{13} \mathrm{C}$ NMR $\left(125 \mathrm{MHz}, \mathrm{CDCl}_{3}\right)$ spectrum of $\mathbf{2 p .}$ 


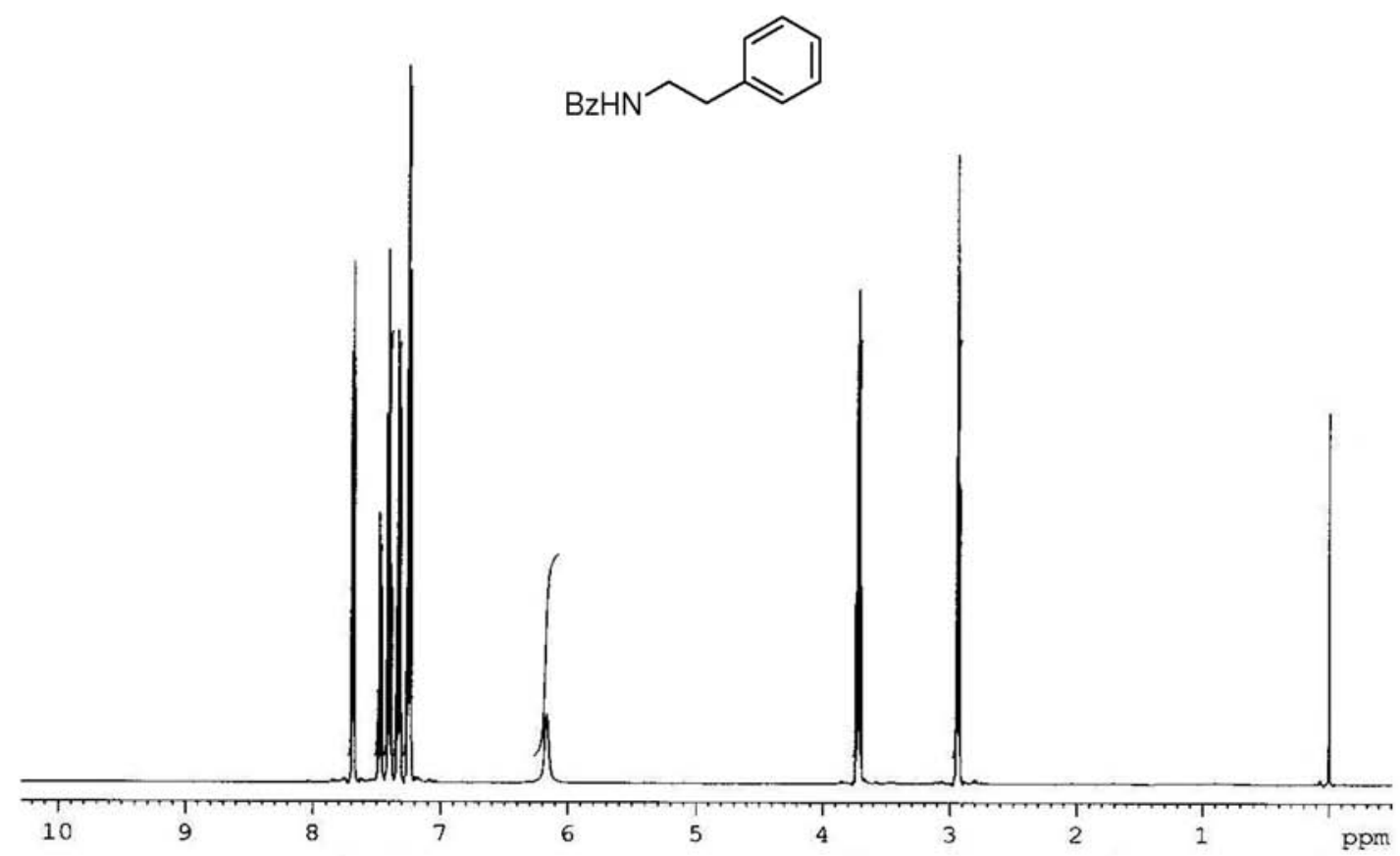

Figure S51. ${ }^{1} \mathrm{H}$ NMR $\left(500 \mathrm{MHz}, \mathrm{CDCl}_{3}\right)$ spectrum of $\mathbf{3 a}$.

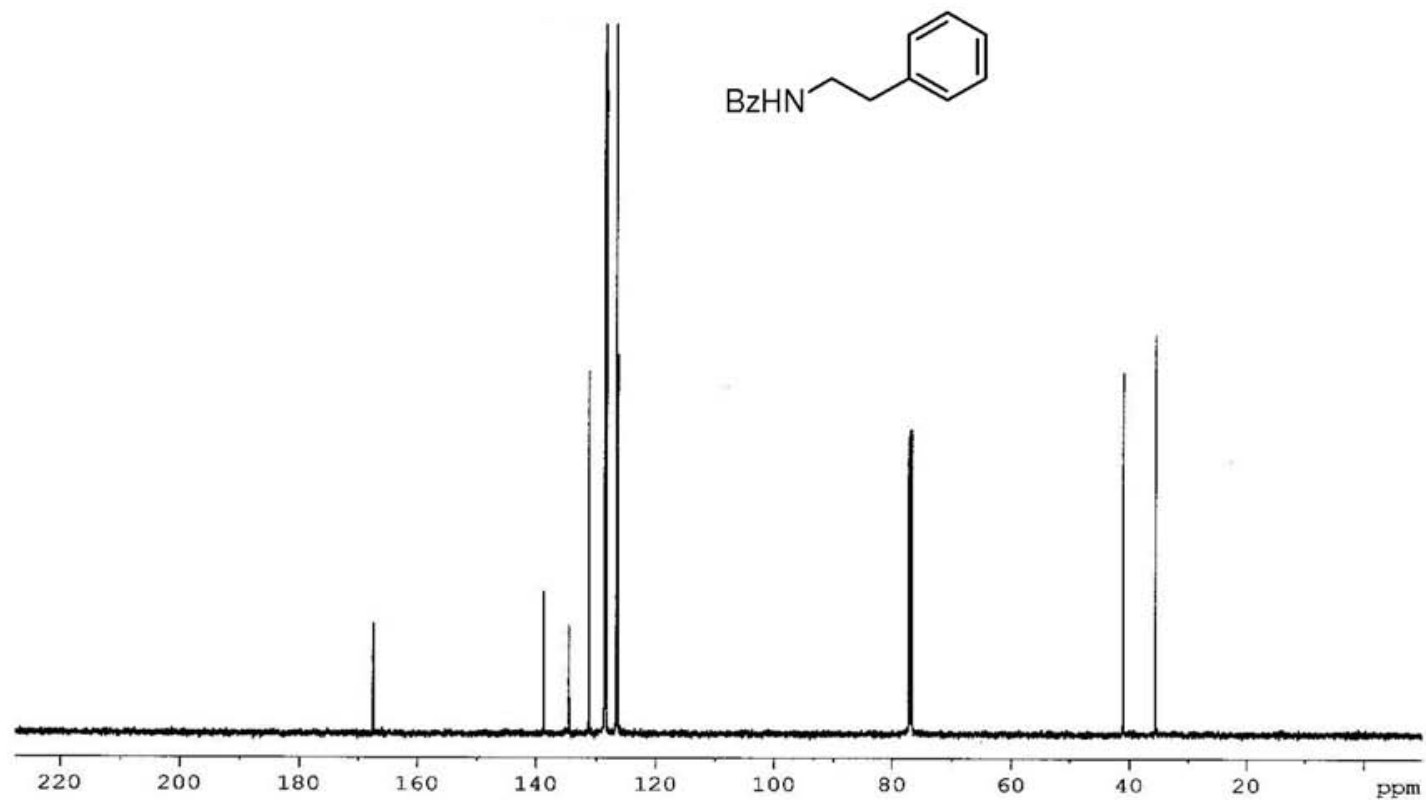

Figure S52. ${ }^{13} \mathrm{C}$ NMR $\left(125 \mathrm{MHz}, \mathrm{CDCl}_{3}\right)$ spectrum of $\mathbf{3 a}$. 


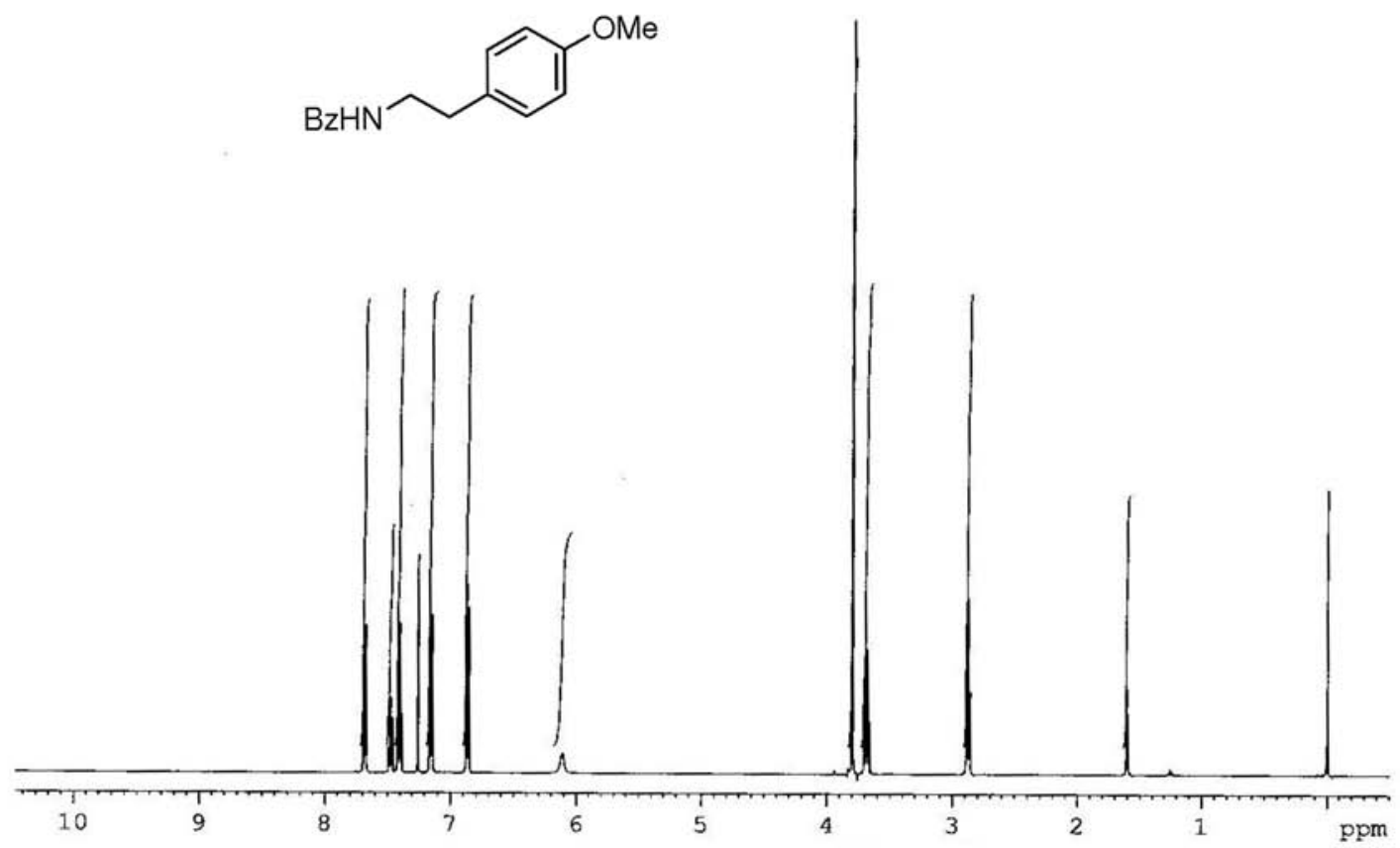

Figure S53. ${ }^{1} \mathrm{H}$ NMR $\left(500 \mathrm{MHz}, \mathrm{CDCl}_{3}\right)$ spectrum of $2 \mathbf{b}$.

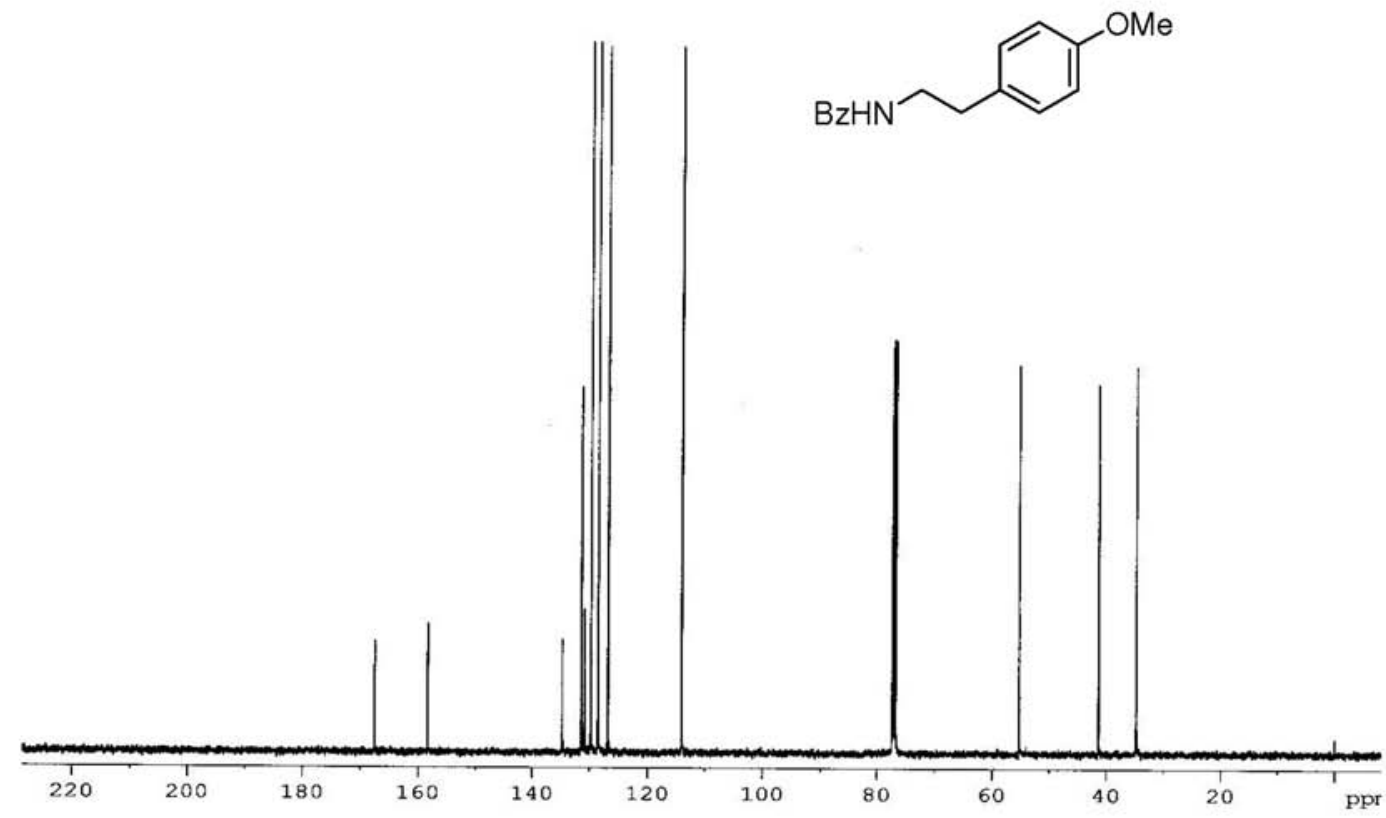

Figure S54. ${ }^{13} \mathrm{C}$ NMR $\left(125 \mathrm{MHz}, \mathrm{CDCl}_{3}\right)$ spectrum of $\mathbf{2 b}$. 


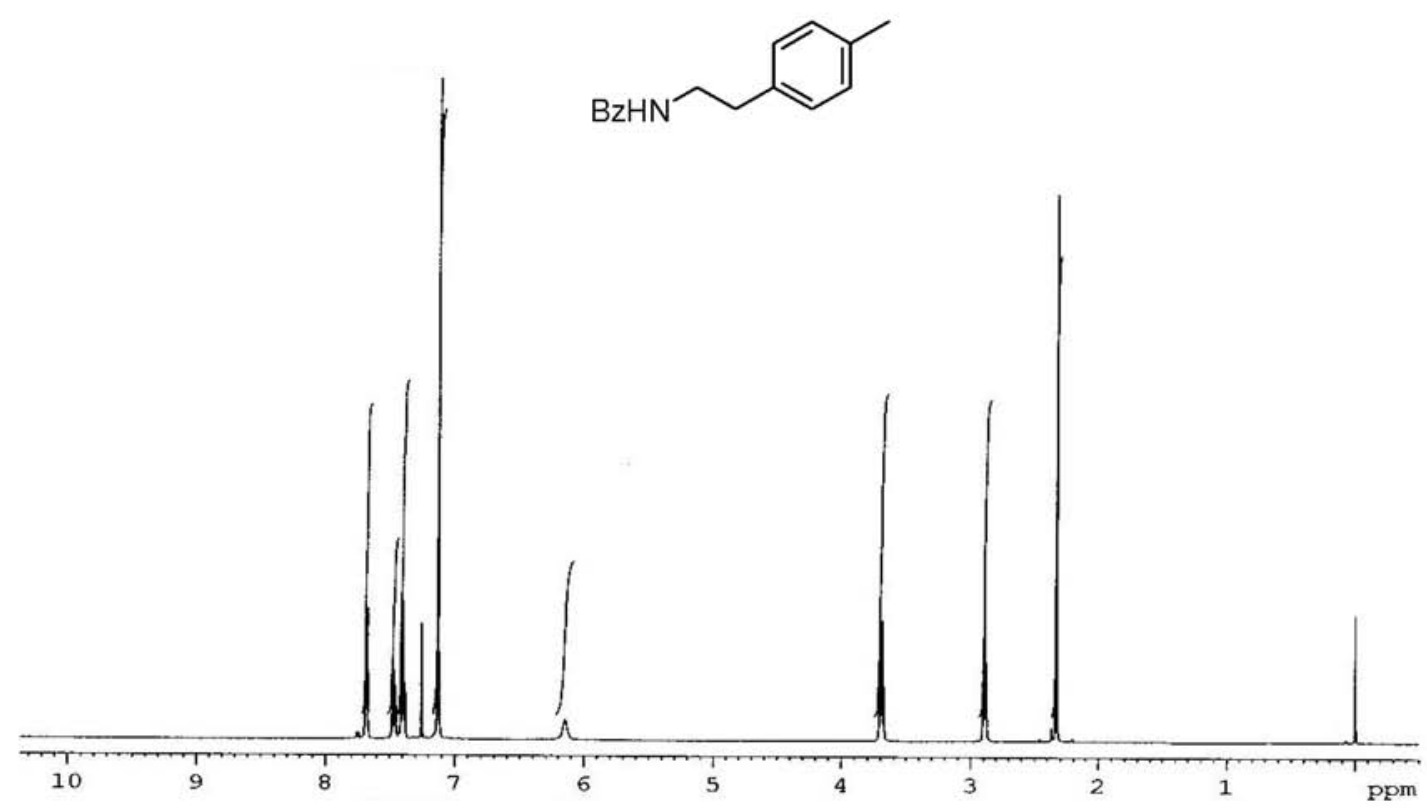

Figure S55. ${ }^{1} \mathrm{H} \mathrm{NMR}\left(500 \mathrm{MHz}, \mathrm{CDCl}_{3}\right)$ spectrum of $\mathbf{3 c}$.
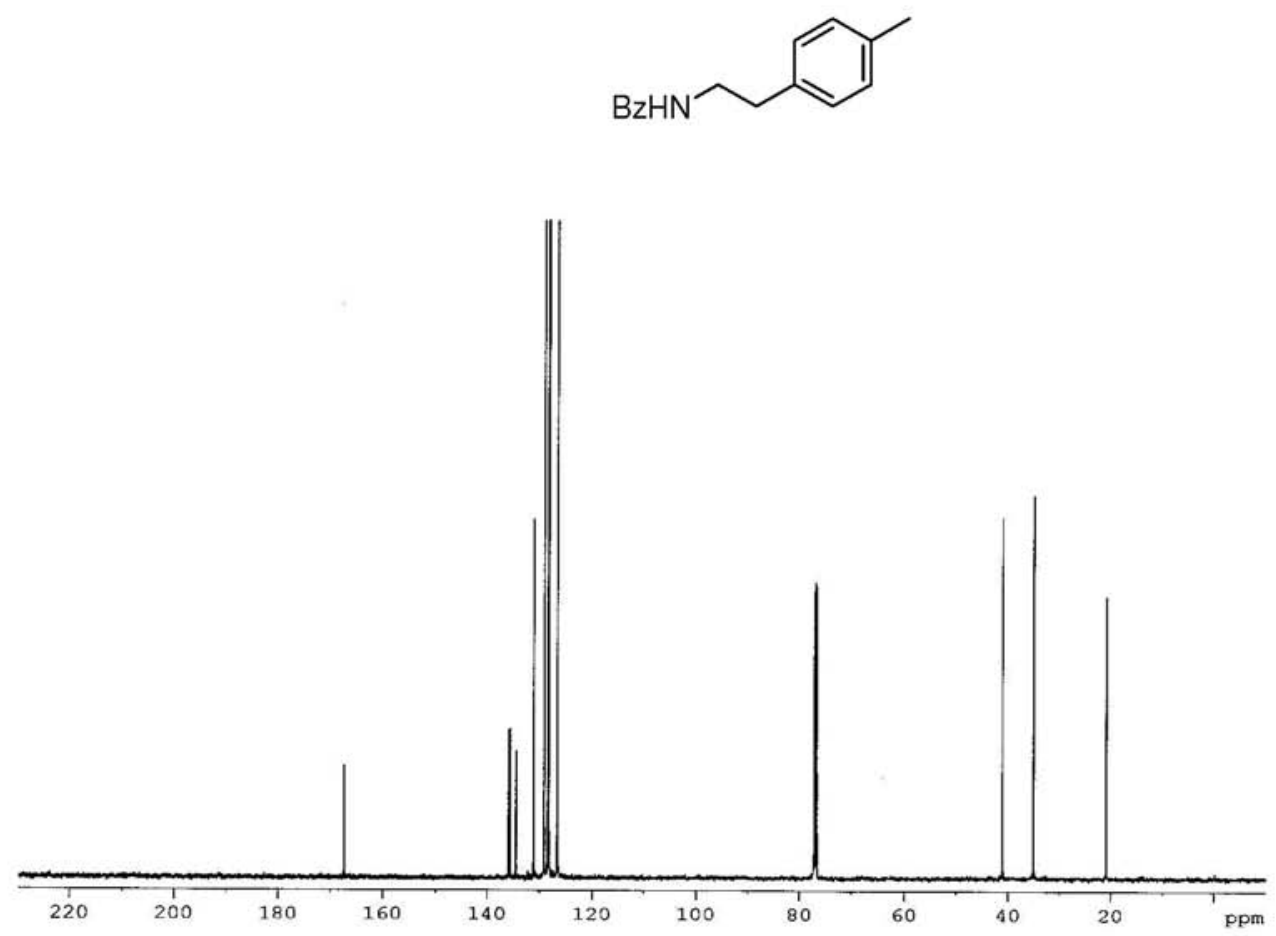

Figure S56. ${ }^{13} \mathrm{C}$ NMR $\left(125 \mathrm{MHz}, \mathrm{CDCl}_{3}\right)$ spectrum of 3c. 


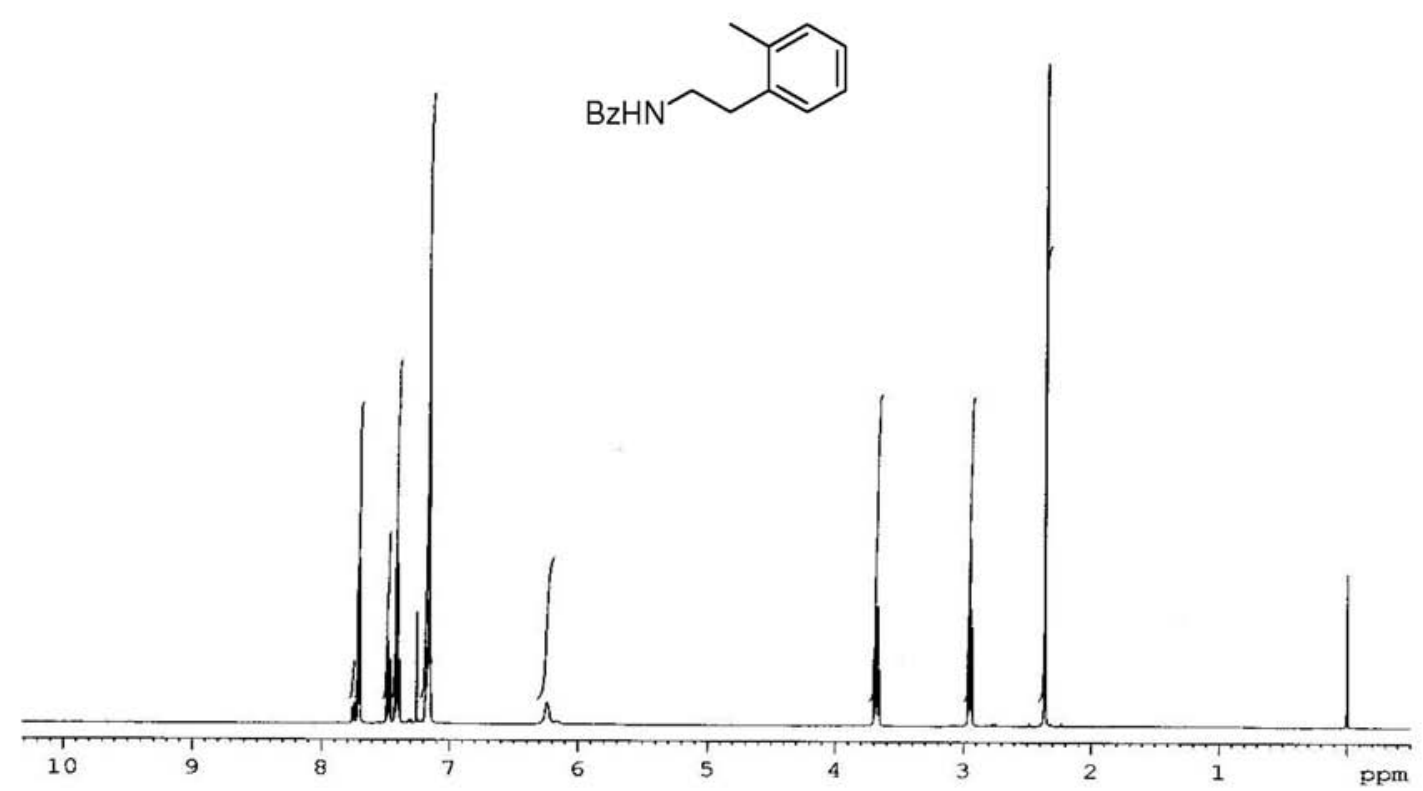

Figure S57. ${ }^{1} \mathrm{H}$ NMR $\left(500 \mathrm{MHz}, \mathrm{CDCl}_{3}\right)$ spectrum of $\mathbf{3 d}$.

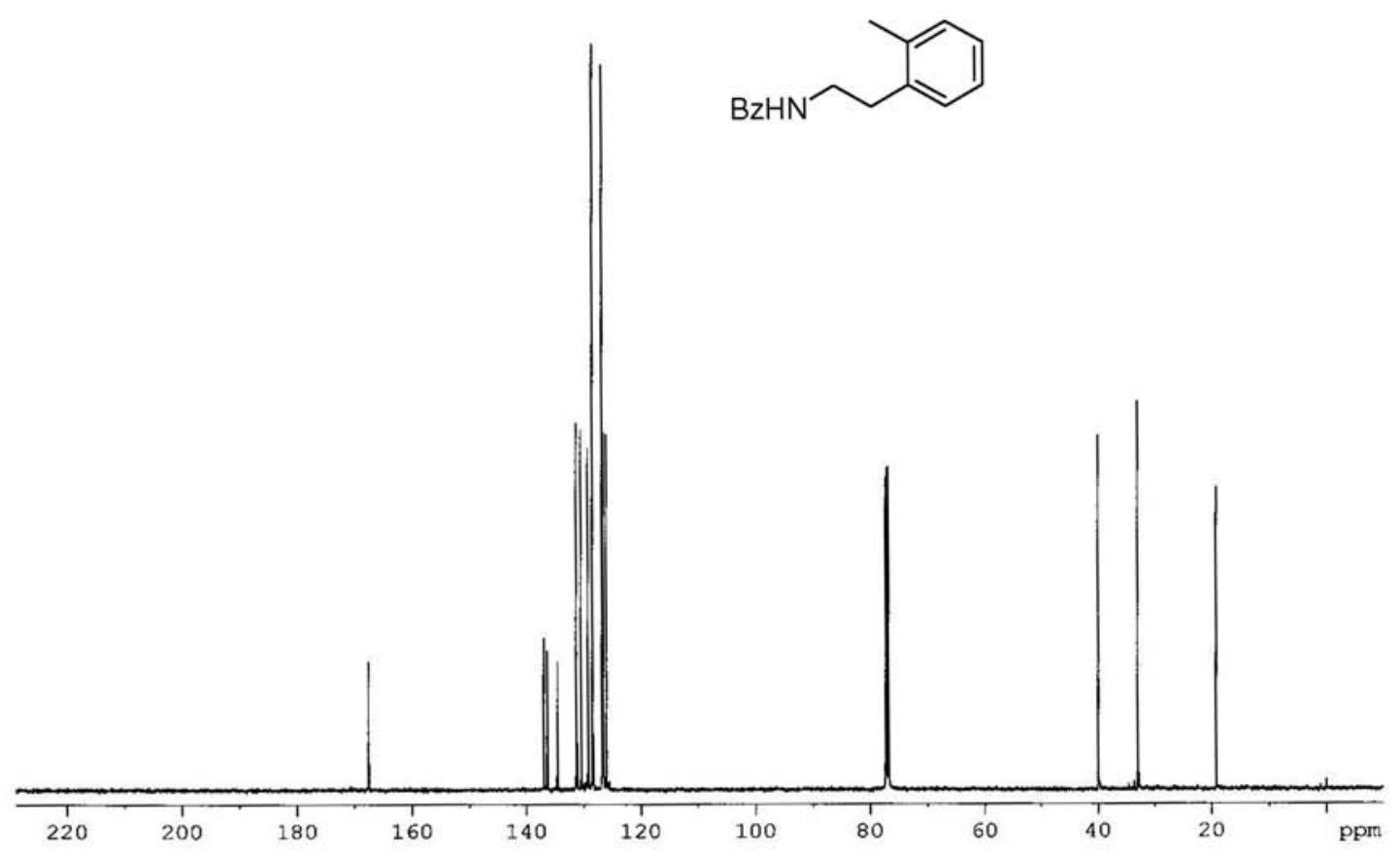

Figure S58. ${ }^{13} \mathrm{C}$ NMR $\left(125 \mathrm{MHz}, \mathrm{CDCl}_{3}\right)$ spectrum of 3d. 


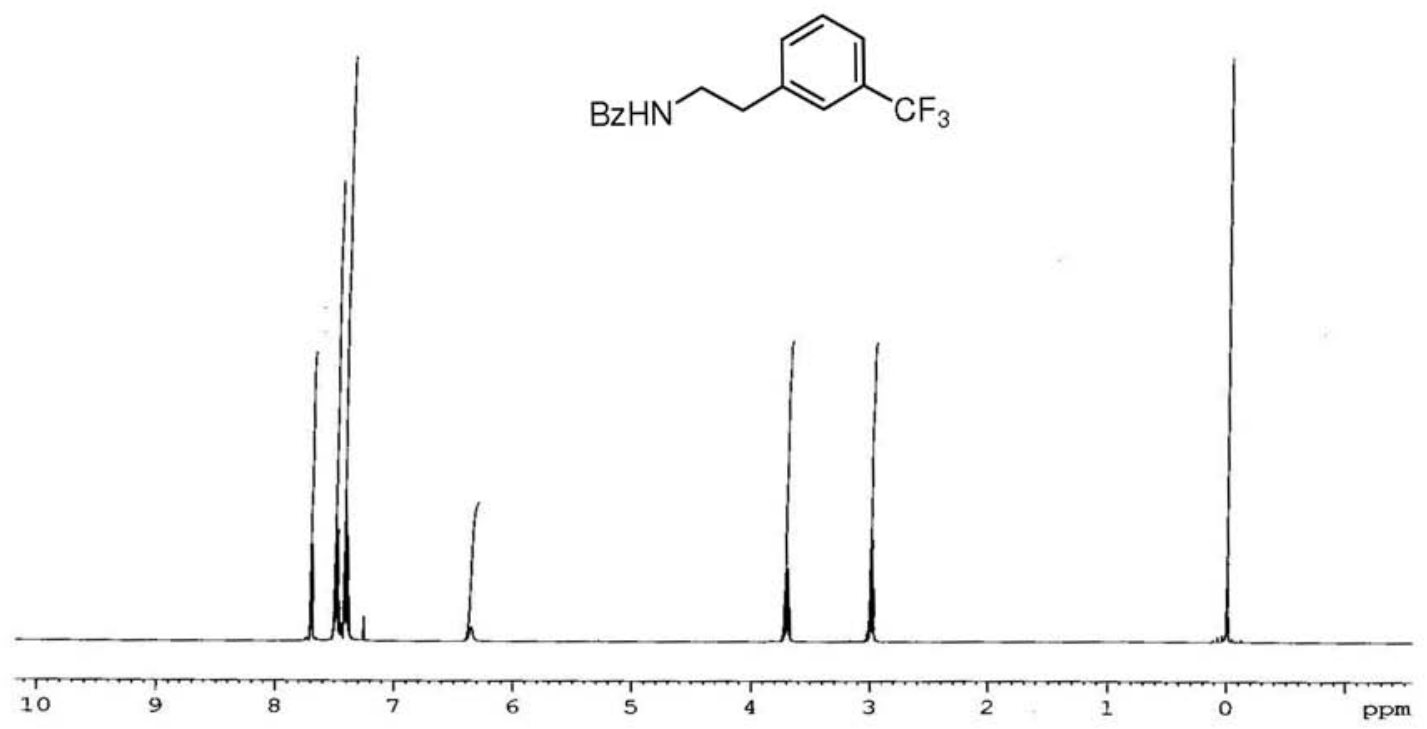

Figure S59. ${ }^{1} \mathrm{H} \mathrm{NMR}\left(500 \mathrm{MHz}, \mathrm{CDCl}_{3}\right)$ spectrum of $\mathbf{3 e}$.

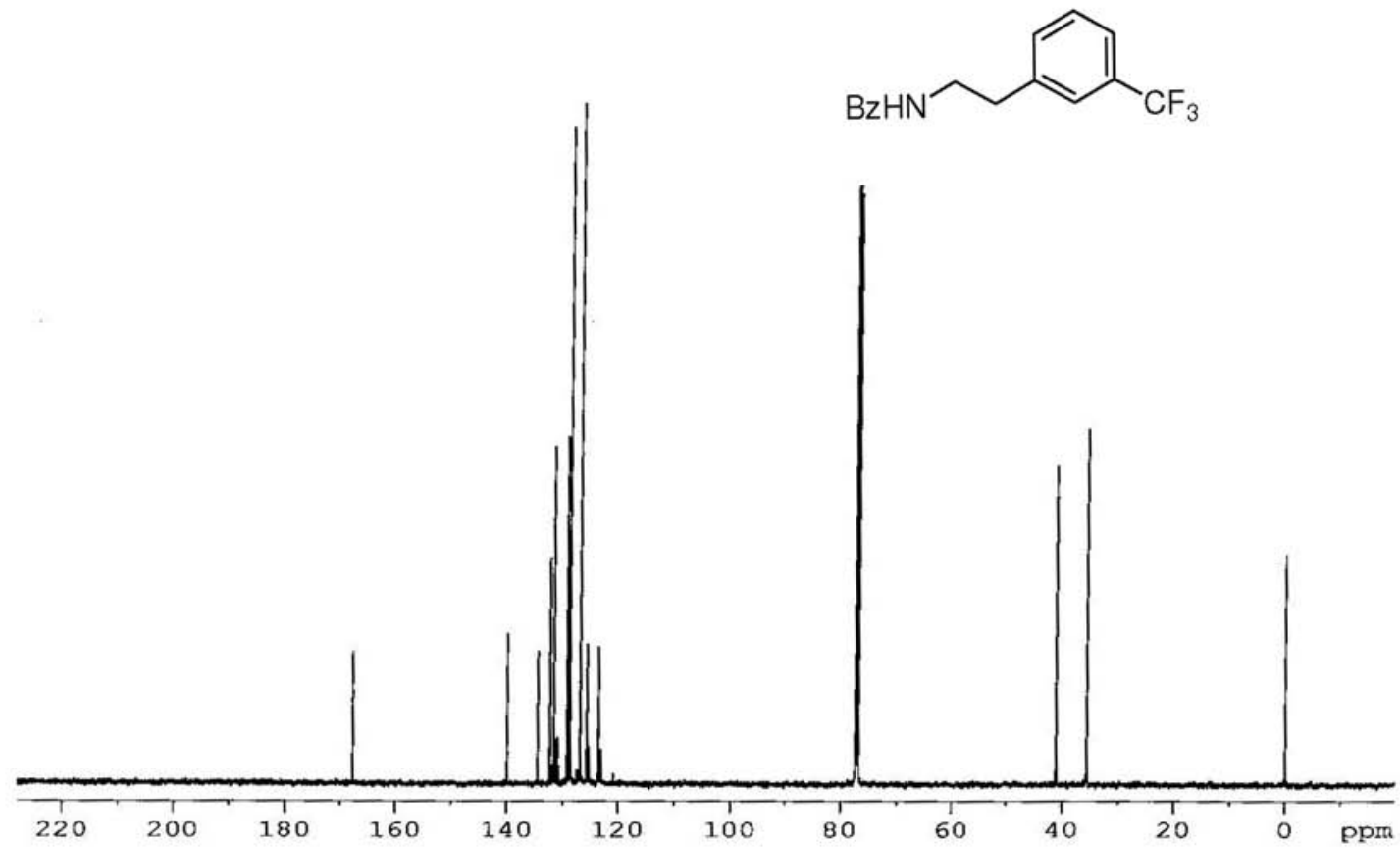

Figure S60. ${ }^{13} \mathrm{C}$ NMR $\left(125 \mathrm{MHz}, \mathrm{CDCl}_{3}\right)$ spectrum of $\mathbf{3 e}$. 
<smiles>O=C(NCCCc1ccccc1)c1ccccc1</smiles>
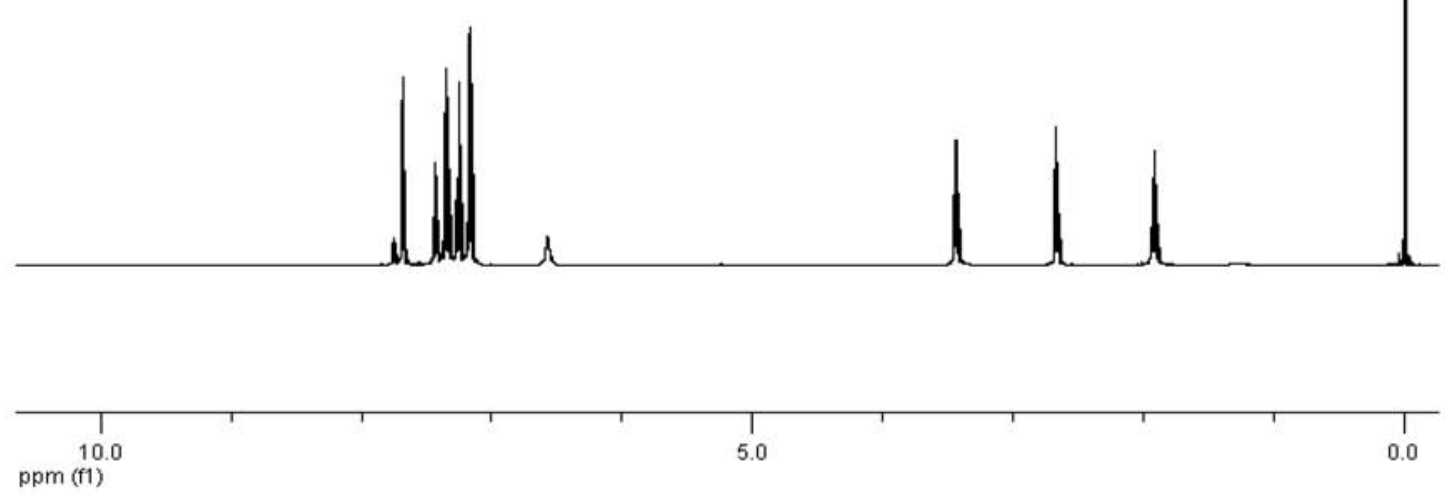

Figure S61. ${ }^{1} \mathrm{H}$ NMR $\left(500 \mathrm{MHz}, \mathrm{CDCl}_{3}\right)$ spectrum of $\mathbf{3 f}$.
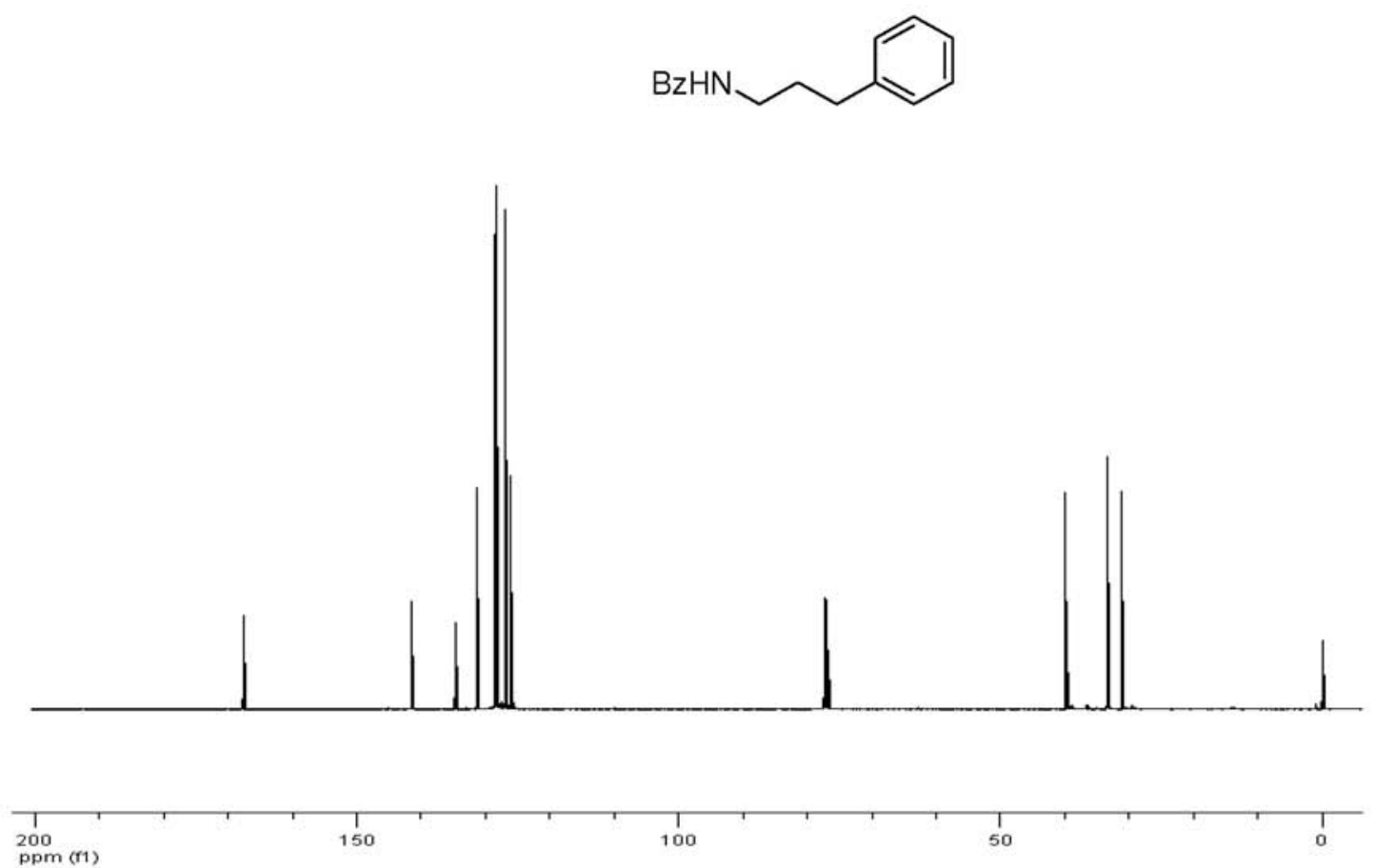

Figure S62. ${ }^{13} \mathrm{C}$ NMR $\left(125 \mathrm{MHz}, \mathrm{CDCl}_{3}\right)$ spectrum of $\mathbf{3 f}$. 